\title{
Discretization of Backward Stochastic Volterra Integral Equations
}

\author{
Dissertation \\ zur Erlangung des Grades \\ des Doktors DeR NAturwissenschaften \\ der Naturwissenschaftlich-Technischen Fakultät I \\ der Universität des Saarlandes
}

von

StANislaV PokAlyuk

SAARBRÜCKEN

2012 
Tag des Kolloquiums: 18.06.2012

Dekan: Univ.-Prof. Dr. Mark Groves

Gutachter: Univ.-Prof. Dr. Christian Bender

Univ.-Prof. Dr. Ralf Korn

Vorsitzender: Univ.-Prof. Dr. Martin Fuchs

Akademischer Beisitzer : Dr. Darya Apushkinskaya 


\section{Eidesstattliche Versicherung}

Hiermit versichere ich an Eides statt, dass ich die vorliegende Arbeit selbstständig und ohne Benutzung anderer als der angegebenen Hilfsmittel angefertigt habe. Die aus anderen Quellen oder indirekt übernommenen Daten und Konzepte sind unter Angabe der Quelle gekennzeichnet. Die Arbeit wurde bisher weder im In- noch im Ausland in gleicher oder ähnlicher Form in einem Verfahren zur Erlangung eines akademischen Grades vorgelegt.

Saarbrücken, 19.04.2012

Stanislav Pokalyuk 


\section{Abstract}

The aim of this thesis is to generalize a numerical method, presented in [18], to solve the stochastic differential equation of Itô's type of the form

$$
Y_{t}=f(W ; t)-\int_{t}^{T} h\left(s, Y_{s}\right) d s-\int_{t}^{T} Z_{t, s} d W_{s}, t \in[0, T],
$$

defined on a filtered probability space $(\Omega, \mathcal{F}, \mathbb{F}, \mathbb{P})$, where $W$ is a $d$-dimensional Brownian motion and $\mathbb{F}$ the corresponding augmented Brownian filtration. Equation (1) is called a backward stochastic Volterra integral equation (BSVIE, for short) firstly introduced in general form in [33], $f$ and $h$ are called the free term and the generator, respectively, of (1).

We show that under certain regularity conditions the solution of (1) can be approximated by the sequence of discrete BSVIEs

$$
Y_{t_{i}}^{(n)}=f\left(W^{(n)} ; t_{i}\right)-\sum_{j=i}^{n-1} h\left(t_{j}, Y_{t_{j}}^{(n)}\right) \Delta t_{j+1}-\sum_{j=i}^{n-1} Z_{t_{i}, t_{j}}^{(n)} \Delta W_{t_{j+1}}^{(n)}
$$

with solutions $\left(Y^{(n)}, Z^{(n)}\right)_{n \in \mathbb{N}}$. In particular, we will prove that the sequence of discrete solutions $\left(Y^{(n)}\right)_{n \in \mathbb{N}}$ converges weakly to the continuous process $Y$ in the Skorokhod topology. As a main tool for the proof we relate the M-solution of (1) to a non-standard system of quasilinear partial differential equations of parabolic type.

Finally, we illustrate the convergence result by a numerical example. 


\section{Zusammenfassung}

Das Ziel dieser Arbeit ist ein numerisches Verfahren, eingeführt in [18], zu verallgemeinern, um stochastische Differentialgleichungen vom Itô Typ, definiert auf einem filtrierten Wahrscheinlichkeitsraum $(\Omega, \mathcal{F}, \mathbb{F}, \mathbb{P})$, von der Form

$$
Y_{t}=f(W ; t)-\int_{t}^{T} h\left(s, Y_{s}\right) d s-\int_{t}^{T} Z_{t, s} d W_{s}, t \in[0, T],
$$

zu lösen, wobei $W$ eine $d$-dimensionale Brownsche Bewegung und $\mathbb{F}$ die zugehörige erweiterte Brownsche Filtration ist. Gleichung (3) nennt man rückwärts stochastische Volterra Integralgleichung (kurz BSVIE), die in allgemeiner Form in 33 . definiert wurde. Dabei nennt man $f$ und $h$ den freien Term, bzw., den Generator von (3).

Wir zeigen, dass unter gewissen Glattheitsbedingungen die Lösung von (3) durch eine Folge von diskreten BSVIEs

$$
Y_{t_{i}}^{(n)}=f\left(W^{(n)} ; t_{i}\right)-\sum_{j=i}^{n-1} h\left(t_{j}, Y_{t_{j}}^{(n)}\right) \Delta t_{j+1}-\sum_{j=i}^{n-1} Z_{t_{i}, t_{j}}^{(n)} \Delta W_{t_{j+1}}^{(n)}
$$

mit Lösungen $\left(Y^{(n)}, Z^{(n)}\right)_{n \in \mathbb{N}}$ approximiert werden kann. Insbesondere, zeigen wir, dass die Folge von diskreten Lösungen $\left(Y^{(n)}\right)_{n \in \mathbb{N}}$ schwach gegen den stetigen Prozess $Y$ in der Skorokhod Topologie konvergiert. Für den Beweis wird es wichtig sein, dass wir die M-Lösung von (3) mit quasilinearen partiellen Differentialgleichungen vom parabolischem Typ verknüpfen können.

Zum Schluß werden wir das Konvergenzresultat noch mit einem numerischen Beispiel veranschaulichen. 


\section{Acknowledgements}

First of all I want to express my deep gratitude to my supervisor Christian Bender, who gave me the opportunity to write this thesis. I am very thankful for his invaluable guidance and encouragement which he has provided through this dissertation, without which, the completion of this work would be uncertain.

Also I would like to thank Ralf Korn who agreed to be as a referee for this thesis.

I would like to acknowledge the support of all my colleagues in Braunschweig and Saarbrücken who have helped me in many situations living in Germany.

Last but not least, I am very grateful to my wife Cornelia and family for their constant support and patience. 


\section{Contents}

Abstract

Zusammenfassung vii

Acknowledgements ix

List of Figures Xiii

Introduction

1 Preliminaries 1

1.1 Basic Notations . . . . . . . . . . . . . . . . . 1

1.2 From BSDEs to BSVIEs . . . . . . . . . . . . . . . . 9

$1.2 .1 \quad$ BSDEs . . . . . . . . . . . . . . . . 9

1.2 .2 BSVIEs . . . . . . . . . . . . . . . . . . 11

1.3 Main Result of this Thesis . . . . . . . . . . . . . . 14

2 Proofs 21

2.1 Proof of Lemma $1.3 .3 \ldots \ldots \ldots$

2.2 Well-posedness of Discrete BSVIEs . . . . . . . . . . . . . . 22

2.3 Estimations for BSVIEs and DBSVIEs . . . . . . . . . . . 28

2.4 Tightness . . . . . . . . . . . . . . . . . 30

2.5 Weak Convergence in Special Case . . . . . . . . . . . . . . 34

2.5.1 Construction for the Solutions of BSVIEs with PDEs . . . 35

2.5.2 Regularity Problem for the Solutions of PDEs . . . . . . . 42

2.5.3 Proof of Lemma 2.5.1 . . . . . . . . . . . . . . . 48

2.6 Proof of the Main Convergence Theorem . . . . . . . . . 52

3 Numerical Example

4 Outlook 
Bibliography

61 


\section{List of Figures}

1.1 Subsets in the Proof of the Well-posedness Theorem for a General BSVIE . . . . . . . . . . . . . . . 13

2.1 Subsets in the Proof of the Well-posedness Theorem for a DBSVIE 23

2.2 Subsets in the Construction for the Solutions of BSVIEs with PDEs 36

2.3 The Algorithm of the Construction Solutions of BSVIEs via Solutions of PDEs . . . . . . . . . . . . 41

3.1 The Speed of Convergence of the Numerical Algorithm . . . . . 57 
LIST OF FIGURES 


\section{Introduction}

In the theory of stochastic differential equations (SDEs, for short) numerical methods, allowing to solve these equations, play an important role, because in most cases it is impossible to obtain explicit solutions for SDEs.

In this thesis we generalize a numerical method originally designed for backward stochastic differential equations (BSDEs, for short) to solve backward stochastic Volterra integral equations (BSVIE, for short) of the form

$$
Y_{t}=f(W ; t)-\int_{t}^{T} h\left(s, Y_{s}\right) d s-\int_{t}^{T} Z_{t, s} d W_{s}, t \in[0, T] .
$$

Remark that the BSVIE (5) differs similar as a BSDE from a forward SDE in the following main features: instead of an initial condition, it has a terminal condition started at time $T$ (namely, $Y_{T}=f(W ; T)$ ), and the solution of (5) is a pair of processes $(Y, Z)$.

The theory of BSDEs and BSVIEs is a relatively modern part of the theory of stochastic differential equations. Intensive research on BSDEs started in the 90s when the well-posedness results were established (see [22]) and the connection between BSDEs and partial differential equations (PDEs, for short) - in fact a generalization of the well-known Feynman-Kac formula to quasilinear PDEs was understood (see [23], 24]).

In mathematical finance the theory of BSDEs plays an important role, when questions are focused around pricing and optimal hedging problems for contingent claims in models of financial markets; see for general information [10]. Further, BSDEs can also be used to solve utility maximization problems with backward stochastic dynamics or to describe dynamic risk measures (see [10, 25, 26, 29]), etc.

If one considers a family of BSDEs parameterized in time, one arrives at the so called backward stochastic Volterra integral equation. In the general form these equations cannot be reduced to BSDEs. We will introduce these equations in Subsection 1.2.2.

The first works dedicated to stochastic Volterra integral equations were presented by Berger and Mizel in 1980 ([4]). In 2002, Lin introduced a class of 


\section{INTRODUCTION}

nonlinear BSVIEs in [17, where he proved the existence and uniqueness of the solutions to these BSVIEs under uniform Lipschitz conditions on the generator.

Thereafter, Yong ([33]) formulated BSVIEs in a generalized form and described their connections to stochastic optimal control theory. In [35] he proposed the well-posedness of adapted M-solutions for these types of BSVIEs (see Theorem 1.2.9 and mentioned in addition about applications of BSVIEs in mathematical finance and risk management (see also [30, 31, 34]).

There are several attempts to provide numercial methods to solve BSDEs, which we can basically be divided into two types:

The first type is based on a four step scheme to solve general forward-backward stochastic differential equations via solutions of quasilinear parabolic PDEs proposed in 1994 by Ma, Protter and Yong ([19]). In 1996 Douglas, Ma and Protter ([8]) and in 2006 Milstein and Tretyakov ([20]) developed two numerical methods using this scheme by approximating numerically the solutions of the corresponding parabolic PDEs.

Bally ([2]) and Chevance ([7]) developed another approach for a numerical solution based on a random time discretization which requires strong regularity conditions. In the same spirit are the papers by Ma, Protter, San Martin, Torres ([18]), and by Briand, Delyon and Memin ([6]), where, in addition, the Brownian motion is replaced by a binary random walk.

So far numerical methods for BSVIEs are missing. Here, we want to close this lack and present an approximative scheme for the solutions of BSVIEs which generalizes the results from [18]. Actually, we approximate the solution of (5) by the sequence of discrete BSVIEs (DBSVIEs, for short)

$$
Y_{t_{i}}^{(n)}=f\left(W^{(n)} ; t_{i}\right)-\sum_{j=i}^{n-1} h\left(t_{j}, Y_{t_{j}}^{(n)}\right) \Delta t_{j+1}-\sum_{j=i}^{n-1} Z_{t_{i}, t_{j}}^{(n)} \Delta W_{t_{j+1}}^{(n)}
$$

with solutions $\left(Y^{(n)}, Z^{(n)}\right)_{n \in \mathbb{N}}$. In particular, we will show that the sequence of discrete solutions $\left(Y^{(n)}\right)_{n \in \mathbb{N}}$ converges weakly to the continuous process $Y$ in the Skorokhod topology. As a main argument we use that certain systems of quasilinear PDEs of parabolic type, to which the M-solution of (5) can be connected, are well approached by its discrete analogs under sufficient regularity constraints.

The rest of the thesis we organize as follows:

Chapter 1 is an introduction to the theory of stochastic analysis needed for our research problem (Section 1.1). Besides, in this chapter we present the wellposedness results for BSDEs and BSVIEs (Section 1.2), and formulate the main result of this thesis (Section 1.3).

In Chapter 2 it is shown Lemma 1.3.3, in which we construct implicit equations for the discrete process $Z^{(n)}$, and proven the Main Convergence Theorem 1.3.6. 
stating the weak convergence of the sequence of discrete solutions $\left(Y^{(n)}\right)_{n \in \mathbb{N}}$ to the continuous process $Y$ in the Skorokhod topology. The proof of Theorem 1.3.6 is divided into five sections. In the second and third section of Chapter 2 we show that the proposed DBSVIEs (6) are well-posed and give an estimate for the difference of two solutions of BSVIEs (DBSVIEs, resp.) depending on the generator and free term. In the fourth section we prove the tightness of the sequence $\left(Y^{(n)}\right)_{n \in \mathbb{N}}$. In the fifth section we show the weak convergence of the sequence $\left(Y^{(n)}\right)_{n \in \mathbb{N}}$ to $Y$, if $f$ does not depend on the whole trajectory of the Brownian motion but only on finitely many points and both, the generator and free term, are smooth. This convergence relies on the fact, that in this case the solution $(Y, Z)$ can be constructed via solutions of systems of PDEs of parabolic type. Together with some lemmas from Section 2.3 we can show the convergence in the general case in Section 2.6.

In Chapter 3 we illustrate the numerical approximation with an example. Here we also obtain the speed of convergence of our algorithm.

In the final Chapter 4 we summarize the results of this thesis and state some open questions for further research. 
INTRODUCTION 


\section{Chapter 1}

\section{Preliminaries}

In this chapter we briefly introduce the main concepts from stochastic analysis which are the basement for our research problem in this thesis. In Section 1.1 we present some well-known definitions and basic results from the theory of probability. Section 1.2 is devoted to the well-posedness problems for backward stochastic differential equations (BSDEs, for short) and backward stochastic Volterra integral equations (BSVIE, for short), which are the main research object of this work. At last, in Section 1.3 we formulate the main result of this thesis.

\subsection{Basic Notations}

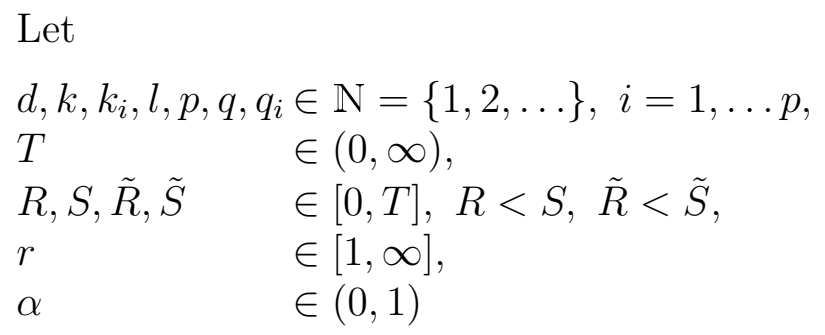

be some variables.

\section{Function spaces}

We use the following standard notations for the function spaces:

$\mathbb{R}_{+}$

the space of all non-negative real numbers.

$\mathbb{R}^{k}$

$k$-dimensional real space with the Euclidean norm $\|\mathbf{x}\|_{2}:=\sqrt{\mathbf{x}^{*} \mathbf{x}}$ and inner product $\langle\mathbf{x}, \mathbf{y}\rangle:=\mathbf{x}^{*} \mathbf{y}$, where $[\cdot]^{*}$ denotes matrix/vektor transposition, $\mathbf{x}:=\left(x_{1}, \ldots, x_{k}\right)^{*}, \mathbf{y}:=\left(y_{1}, \ldots, y_{k}\right)^{*} \in \mathbb{R}^{k}$. 


\section{PRELIMINARIES}

$\mathbb{R}^{k \times d}$

$C\left([R, S] ; \mathbb{R}^{k}\right)$

$C^{\infty}\left(X_{1} \times \ldots \times X_{p} ; \mathbb{R}^{k}\right)$

$C_{b}^{\infty}\left(X_{1} \times \ldots \times X_{p} ; \mathbb{R}^{k}\right)$ the Hilbert space of all $(k \times d)$ real matrices with the Euclidean norm $\|A\|_{2}:=\sqrt{\operatorname{tr}\left(A A^{*}\right)}$ and inner product $\langle A, B\rangle:=\operatorname{tr}\left(A B^{*}\right), A, B \in \mathbb{R}^{k \times d}$.

the space of all continuous functions $\varphi:[R, S] \rightarrow$ $\mathbb{R}^{k}$ with the norm $\|\varphi\|_{\infty}:=\sup _{t \in[R, S]}\|\varphi(t)\|_{2}$.

the space of all smooth functions $\varphi: X_{1} \times \ldots \times$ $X_{p} \rightarrow \mathbb{R}^{k}$, such that $X_{i}=\mathbb{R}$ or $X_{i}=[R, S], i=$ $1, \ldots, p$.

the space of those $\varphi \in C^{\infty}\left(X_{1} \times \ldots \times X_{p} ; \mathbb{R}^{k}\right)$ such that all derivatives are uniformly bounded.

\section{Probability spaces and random variables}

Let $(\Omega, \mathcal{F}, \mathbb{P})$ be a probability space, where $\Omega$ is a nonempty set of elementary events $\omega, \mathcal{F} \subseteq 2^{\Omega}$ ( $2^{\Omega}$ is the set of all subsets in $\Omega$ ) is a $\sigma$-field on $\Omega$ and the map $\mathbb{P}: \mathcal{F} \rightarrow[0,1]$ is a probability measure on the measurable space $(\Omega, \mathcal{F})$. We assume that $(\Omega, \mathcal{F}, \mathbb{P})$ is complete, i.e. if $A \in \mathcal{F}, \mathbb{P}[A]=0$ and $B \subset A$ then it holds that also $B \in \mathcal{F}$.

Any $A \in \mathcal{F}$ is called an event. We say that an event $A$ holds $\mathbb{P}$-a.s. (a.s. $=$ almost surely) if $\mathbb{P}[A]=1$; if $\mathbb{P}[A]=0$ then $A$ is called $\mathbb{P}$-null event. Denote by $\sigma(\mathcal{A})$ the smallest $\sigma$-field containing $\mathcal{A}(\sigma$-field generated by $\mathcal{A})$, for any $\mathcal{A} \subseteq 2^{\Omega}$.

Considering stochastic processes we need to specify on the probability space a filtration $\mathbb{F}:=\left(\mathcal{F}_{t}\right)_{t \in[0, T]}$, i.e. $\left(\mathcal{F}_{t}\right)_{t \in[0, T]}$ is a family of sub- $\sigma$-fields $\mathcal{F}_{t} \subseteq \mathcal{F}$ with $\mathcal{F}_{t_{1}} \subseteq \mathcal{F}_{t_{2}}, \forall 0 \leq t_{1} \leq t_{2} \leq T$. Under $\mathcal{F}_{t}$ one understands the collection of events observed up to the moment $t$. We say that $\mathbb{F}$ is right continuous if

$$
\mathcal{F}_{t}=\bigcap_{s>t} \mathcal{F}_{s}
$$

for all $t \in[0, T]$. We call $\mathbb{F}$ complete if $\mathcal{F}_{0}$ contains all the $\mathbb{P}$-null events in $\mathcal{F}$. Note that a filtration $\mathbb{F}$ can be made complete by augmentation procedure, i.e. denoting by

$$
\mathcal{N}:=\{A \in \mathcal{F}: \mathbb{P}[A]=0\}
$$

the set of $\mathbb{P}$-null events in $\mathcal{F}$, we define $\hat{\mathcal{F}}_{t}:=\sigma\left(\mathcal{F}_{t} \cup \mathcal{N}\right)$ and $\hat{\mathbb{F}}:=\left(\hat{\mathcal{F}}_{t}\right)_{t \in[0, T]}$. Then it holds that $(\Omega, \mathcal{F}, \hat{\mathbb{F}}, \mathbb{P})$ is a complete filtered probability space. We call $\hat{\mathbb{F}}$ the augmented filtration of $\mathbb{F}$. 
Throughout this work we consider the filtered probability space $(\Omega, \mathcal{F}, \mathbb{F}, \mathbb{P})$ satisfying the usual conditions, that is $\mathbb{F}$ is complete and right continuous.

For a topological space $(U, \mathcal{T})$ the smallest $\sigma$-field containing all open sets in $\mathcal{T}$ is called the Borel $\sigma$-field of $(U, \mathcal{T})$ and denoted by $\mathcal{B}_{\mathcal{T}}(U)$, or shortly $\mathcal{B}(U)$.

Further, let $(\Omega, \mathcal{F}),\left(\Omega^{\prime}, \mathcal{F}^{\prime}\right)$ be two measurable spaces. For a $\left(\mathcal{F}, \mathcal{F}^{\prime}\right)$-measurable map $X: \Omega \rightarrow \Omega^{\prime}$ we define by

$$
\sigma(X):=X^{-1}\left(\mathcal{F}^{\prime}\right):=\left\{X^{-1}\left(A^{\prime}\right): A^{\prime} \in \mathcal{F}^{\prime}\right\}
$$

the $\sigma$-field generated by random element $X$. If $\left(\Omega^{\prime}, \mathcal{F}^{\prime}\right)=\left(\mathbb{R}^{k}, \mathcal{B}\left(\mathbb{R}^{k}\right)\right)$ we say that $X$ is $\mathcal{F}$-measurable and $X$ is called a ( $k$-dimensional) random variable.

Similarly, for a family of random elements $\left(X_{i}\right)_{i \in I}$ from $(\Omega, \mathcal{F})$ to $\left(\Omega^{\prime}, \mathcal{F}^{\prime}\right)$ we define

$$
\sigma\left(X_{i}: i \in I\right):=\sigma\left(\bigcup_{i \in I} \sigma\left(X_{i}\right)\right)=\sigma\left(\bigcup_{i \in I} X_{i}^{-1}\left(\mathcal{F}^{\prime}\right)\right)
$$

as the smallest sub- $\sigma$-field of $\mathcal{F}$ under which all $X_{i}$ are $\left(\mathcal{F}, \mathcal{F}^{\prime}\right)$-measurable, $i \in I$.

Next, denote by $\mathbb{P}_{X}$ on $\left(\Omega^{\prime}, \mathcal{F}^{\prime}\right)$ the distribution of the random variable $X$ and by $\mathbb{E}[X]$ the expectation relative to the measure $\mathbb{P}$.

Taking a sub- $\sigma$-field $\mathcal{G} \subseteq \mathcal{F}$ and integrable random variable $X$, i.e. with $\mathbb{E}\left[\left|X_{i}\right|\right]<\infty, i=1, \ldots, k$, we denote by $\mathbb{E}[X \mid \mathcal{G}]$ the conditional expectation of $X$ given $\mathcal{G}$.

\section{Stochastic processes}

We say that a family of random variables $\left(X_{t}\right)_{t \in[0, T]}$ from $(\Omega, \mathcal{F}, \mathbb{P})$ to $\mathbb{R}^{k}$ is a stochastic process on $(\Omega, \mathcal{F}, \mathbb{F}, \mathbb{P})$. We will write $\left(X_{t}\right)_{t \in[0, T]}, X(\cdot)$, or sometimes $X$ to denote a stochastic process.

A stochastic process $\left(X_{t}\right)_{t \in[0, T]}$ is said to be $\mathbb{F}$-adapted if the mapping $\omega \mapsto$ $X_{t}(\omega)$ is an $\mathcal{F}_{t}$-measurable random variable for all $t \in[0, T] . \quad\left(X_{t}\right)_{t \in[0, T]}$ is called $\mathbb{F}$-progressively measurable if the mapping $(\omega, s) \mapsto X_{s}(\omega)$ is $\mathcal{F}_{t} \otimes \mathcal{B}([0, t])$ measurable for $t \in[0, T]$ and $s \in[0, t]$.

The filtration $\mathbb{F}=\left(\mathcal{F}_{t}\right)_{t \in[0, T]}$ is called natural if it is generated by a stochastic process $X$, i.e. $\mathcal{F}_{t}=\sigma\left(X_{s}: s \in[0, t]\right)$.

For any $\omega \in \Omega$, the map $t \mapsto X_{t}(\omega)$ is called a sample path of the process $X$. One says that $X$ has càdlàg paths if $\mathbb{P}$-a.s. the path $t \mapsto X_{t}(\omega)$ is rightcontinuous in $t \in[0, T]$ and there exist left limits in $t>0$. The space of all functions defined on $[0, T]$ with càdlàg paths we will denote by $\mathbb{D}\left([0, T] ; \mathbb{R}^{k}\right)$, also called the Skorokhod space (see [5] for a more general construction).

An $\mathbb{R}$-valued stochastic process $\left(X_{t}\right)_{t \in[0, T]}$ is called a martingale with respect to the filtration $\mathbb{F}$ (shortly, $\mathbb{F}$-martingale), if it is integrable for each $t \in[0, T]$, i.e. 


\section{PRELIMINARIES}

$\mathbb{E}\left[\left|X_{t}\right|\right]<\infty, \mathbb{F}$-adapted with $\mathbb{P}$-almost all càdlàg paths, and it holds $\mathbb{E}\left[X_{t} \mid \mathcal{F}_{s}\right]=$ $X_{s} \mathbb{P}$-a.s. for every $s \in[0, t]$.

Let $(\Omega, \mathcal{F}, \mathbb{F}, \mathbb{P})$ satisfy the usual conditions. An $\mathbb{F}$-adapted stochastic process $\left(X_{t}\right)_{t \in[0, T]}$ with càdlàg paths is called a semimartingale, if it can be represented as a sum

$$
X_{t}=X_{0}+M_{t}+A_{t}
$$

where $\left(M_{t}\right)_{t \in[0, T]}$ is an $\mathbb{F}$-martingale, $\left(A_{t}\right)_{t \in[0, T]}$ is an $\mathbb{F}$-adapted process with paths of finite variation and $M_{0}=A_{0}=0$.

If $\left(X_{t}\right)_{t \in[0, T]}$ is a $\mathbb{R}^{k}$-valued process in the above definitions the conditions must hold for every coordinate.

Further, we define some important spaces:

$$
\begin{aligned}
& \mathbb{L}_{\mathcal{F}_{S}}^{r}\left(\Omega ; \mathbb{R}^{k}\right) \quad \text { the space of } \mathcal{F}_{S^{-}} \text {measurable random variables } X \text { : } \\
& \Omega \rightarrow \mathbb{R}^{k} \text { such that } \mathbb{E}\left[\|X\|_{2}^{r}\right]<\infty \text {. } \\
& \mathcal{L}_{\mathbb{F}}^{2}\left(R, S ; \mathbb{R}^{k}\right) \quad \text { the space of all } \mathbb{F} \text {-adapted processes } Y: \Omega \times \\
& {[R, S] \rightarrow \mathbb{R}^{k} \text { such that } \int_{R}^{S}\|Y(\cdot, t)\|_{2}^{2} d t<\infty, \mathbb{P}_{-}} \\
& \text {a.s. } \\
& \mathbb{L}_{\mathbb{F}}^{2}\left(C([R, S]) ; \mathbb{R}^{k}\right) \quad \text { the space of all } \mathbb{F} \text {-adapted continuous pro- } \\
& \text { cesses } Y: \Omega \times[R, S] \rightarrow \mathbb{R}^{k} \text { such that } \\
& \mathbb{E}\left[\sup _{t \in[R, S]}\|Y(\cdot, t)\|_{2}^{2}\right]<\infty \text {. } \\
& L_{\mathcal{F}_{S}}^{2}\left(R, S ; \mathbb{R}^{k}\right) \quad \text { the space of } \mathcal{F}_{S} \otimes \mathcal{B}([R, S]) \text {-measurable pro- } \\
& \text { cesses } Y: \Omega \times[R, S] \rightarrow \mathbb{R}^{k} \text { such that } \\
& \mathbb{E}\left[\int_{R}^{S}\|Y(\cdot, t)\|_{2}^{2} d t\right]<\infty .
\end{aligned}
$$


$L_{\mathbb{F}}^{2}\left(R, S ; \mathbb{R}^{k}\right)$

$M_{\mathbb{F}}^{2}\left(R, S ; \mathbb{R}^{k}\right)$

$L^{2}\left(R, S ; L_{\mathbb{F}}^{2}\left(\tilde{R}, \tilde{S} ; \mathbb{R}^{k \times d}\right)\right)$

$L_{\mathbb{F}}^{2, \infty}\left(R, S ; \mathbb{R}^{k}\right)$ the space of $\mathcal{F}_{S} \otimes \mathcal{B}([R, S])$-measurable processes $Y: \Omega \times[R, S] \rightarrow \mathbb{R}^{k}$ such that $Y$ is $\mathbb{F}$-adapted and it holds $\mathbb{E}\left[\int_{R}^{S}\|Y(\cdot, t)\|_{2}^{2} d t\right]<\infty$. We define $\|Y\|_{L_{\mathrm{F}}^{2}\left(R, S ; \mathbb{R}^{k}\right)}:=\left(\mathbb{E}\left[\int_{R}^{S}\|Y(\cdot, t)\|_{2}^{2} d t\right]\right)^{\frac{1}{2}}$.

the space of those $Y \in L_{\mathbb{F}}^{2}\left(R, S ; \mathbb{R}^{k}\right)$ such that $Y$ is a martingale with respect to the filtration $\mathbb{F}$ and it holds $Y(\cdot, 0)=0, \mathbb{P}$-a.s.

the space of all processes $Z: \Omega \times[R, S] \times[\tilde{R}, \tilde{S}] \rightarrow$ $\mathbb{R}^{k \times d}$ such that $Z(\cdot, t, \cdot) \in L_{\mathbb{F}}^{2}\left(\tilde{R}, \tilde{S} ; \mathbb{R}^{k \times d}\right)$ for a.e. $t \in[R, S]$ and $\mathbb{E}\left[\int_{R}^{S} \int_{\tilde{R}}^{\tilde{S}}\|Z(\cdot, t, s)\|_{2}^{2} d s d t\right]<$ $\infty$. We define $\|Z\|_{L^{2}\left(R, S ; L_{\tilde{R}}^{2}\left(\tilde{R}, \tilde{S} ; \mathbb{R}^{k \times d}\right)\right)}:=$ $\left(\mathbb{E}\left[\int_{R}^{S} \int_{\tilde{R}}^{\tilde{S}}\|Z(\cdot, t, s)\|_{2}^{2} d s d t\right]\right)^{\frac{1}{2}}$.

the space of $\mathcal{F}_{S} \otimes \mathcal{B}([R, S])$-measurable processes $Y: \Omega \times[R, S] \rightarrow \mathbb{R}^{k}$ such that $Y$ is $\mathbb{F}$-adapted and it holds $\mathbb{E}\left[\sup _{t \in[R, S]}\|Y(\cdot, t)\|_{2}^{2}\right]<\infty$. We define $\|Y\|_{L_{\mathrm{F}}^{2, \infty}\left(R, S ; \mathbb{R}^{k}\right)}:=\left(\mathbb{E}\left[\sup _{t \in[R, S]}\|Y(\cdot, t)\|_{2}^{2}\right]\right)^{\frac{1}{2}}$.

Let on $(\Omega, \mathcal{F}, \mathbb{F}, \mathbb{P})$ the filtration $\mathbb{F}$ be the augmented Brownian filtration and $W_{t}$ be an $d$-dimensional Brownian motion. A $k$-dimensional stochastic process $X_{t}$ such that $X_{0}$ is $\mathcal{F}_{0}$-measurable with $b_{t} \in \mathcal{L}_{\mathbb{F}}^{2}\left(0, T ; \mathbb{R}^{k}\right)$ and $\sigma_{t}=\left(\sigma_{t}^{i j}\right) \in$ $\mathcal{L}_{\mathbb{F}}^{2}\left(0, T ; \mathbb{R}^{k \times d}\right)$ of a form

$$
X_{t}=X_{0}+\int_{0}^{t} b_{s} d s+\int_{0}^{t} \sigma_{s} d W_{s}, \quad t \in[0, T], \mathbb{P} \text {-a.s., }
$$

is called an Itô process, where

$$
X_{t}:=\left(\begin{array}{c}
X_{t}^{1} \\
\vdots \\
X_{t}^{k}
\end{array}\right), W_{t}:=\left(\begin{array}{c}
W_{t}^{1} \\
\vdots \\
W_{t}^{d}
\end{array}\right), \int_{0}^{t} \sigma_{s} d W_{s}:=\left(\begin{array}{c}
\sum_{j=1}^{d} \int_{0}^{t} \sigma_{s}^{1 j} d W_{s}^{j} \\
\vdots \\
\sum_{j=1}^{d} \int_{0}^{t} \sigma_{s}^{k j} d W_{s}^{j}
\end{array}\right)
$$




\section{PRELIMINARIES}

and $\int_{0}^{t} \sigma_{s}^{k j} d W_{s}^{j}$ is an Itô integral. Note that we always deal with an Itô integral; see details in construction and basic properties of Itô integrals, for instance, in [12].

\section{Martingale representation theorem}

Suppose that for some fixed terminal time $T>0$ a standard $d$-dimensional Brownian motion $W_{t}, t \in[0, T]$, is defined on $(\Omega, \mathcal{F}, \mathbb{F}, \mathbb{P})$, where we assume that $\mathbb{F}$ is the augmented Brownian filtration. Then the following theorem holds:

Theorem 1.1.1. (Martingale representation theorem) Assume that $X_{t} \in$ $M_{\mathbb{F}}^{2}\left(0, T ; \mathbb{R}^{k}\right)$. Then there exists a unique process $\varphi_{t} \in L_{\mathbb{F}}^{2}\left(0, T ; \mathbb{R}^{d}\right)$ such that

$$
X_{t}=X_{0}+\int_{0}^{t}\left\langle\varphi_{s}, d W_{s}\right\rangle, \quad \forall t \in[0, T], \mathbb{P} \text {-a.s. }
$$

A proof of the martingale representation theorem can be found, for example, in [15], Chapter 3, Theorem 4.15.

\section{Martingale representation theorem in discrete time}

There is an analogous version of Theorem 1.1.1 for the discrete-time martingales.

Given a discretization $0=t_{0}<t_{1}<\ldots<t_{n}=T$ of the time interval $[0, T]$ with time-step $\frac{T}{n}\left(t_{i}:=\frac{i T}{n}, i=0, \ldots, n, n \in \mathbb{N}\right)$, replace the $d$-dimensional Brownian motion $W_{s}$ in Theorem 1.1.1 by a simple symmetric random walk $W_{s}^{(n)}$, whose increments are $\sqrt{T / n}$ and $-\sqrt{T / n}$; i.e. if the number $n s / T$ is an integer

$$
W_{s}^{(n)}:=\left(\begin{array}{c}
W_{s}^{1,(n)} \\
\vdots \\
W_{s}^{d,(n)}
\end{array}\right):=\sqrt{\frac{T}{n}}\left(\begin{array}{cc}
\sum_{j=1}^{n s / T} \varepsilon_{j}^{1} \\
\vdots \\
\sum_{j=1}^{n s / T} \varepsilon_{j}^{d}
\end{array}\right)
$$

$\left\{\varepsilon_{j}^{d}\right\}$ is an i.i.d. $\{-1,1\}$-symmetric sequence, and if $n s / T$ is not an integer then $W_{s}^{(n)}$ is defined by linear interpolation between the values of $W_{s^{\prime}}^{(n)}$ and $W_{s^{\prime \prime}}^{(n)}$ for which $n s^{\prime} / T$ and $n s^{\prime \prime} / T$ are integers and the nearest points to the left and right of $n s / T$.

By $\mathbb{F}^{(n)}:=\left(\mathcal{F}_{t_{i}}^{(n)}\right)_{i=0, \ldots, n}$ we denote the natural filtration of $W^{(n)}$, where $\mathcal{F}_{t_{i}}^{(n)}:=$ $\sigma\left(W_{t_{i}}^{(n)}: i=0, \ldots, n\right)$.

Then it holds: 
Theorem 1.1.2. (Discrete martingale representation theorem) Assume that $\left(X_{t_{i}}\right)_{i=0, \ldots, n}$ is a martingale in $L_{\mathbb{F}^{(n)}}^{2}\left(t_{0}, t_{n} ; \mathbb{R}^{k}\right)$ (see definition in Section 1.3 ). Then there exists a predictable sequence $\left(\varphi_{t_{i}}\right)_{i=1, \ldots, n}$ (that is $\varphi_{t_{i}}$ are $\left(\mathcal{F}_{t_{i-1}}^{(n)}\right)_{i=1, \ldots, n^{-}}$ measurable) in $L_{\mathbb{F}^{(n)}}^{2}\left(t_{1}, t_{n} ; \mathbb{R}^{d}\right)$ such that

$$
X_{t_{i}}=X_{t_{0}}+\sum_{j=1}^{i}\left\langle\varphi_{t_{j}}, \Delta W_{t_{j}}^{(n)}\right\rangle, \quad i=1, \ldots, n
$$

where $\Delta W_{t_{j}}^{(n)}:=W_{t_{j}}^{(n)}-W_{t_{j-1}}^{(n)}$ with $\Delta W_{t_{j}}^{J,(n)}:=W_{t_{j}}^{J,(n)}-W_{t_{j-1}}^{J,(n)}=\sqrt{\frac{T}{n}} \varepsilon_{j}^{J}$ for all $J \in\{1, \ldots, d\}$.

A proof of the discrete martingale representation theorem can be found, for example, in [27].

\section{Itô's formula}

Here, we would like to recall one of the most important tools in stochastic calculus called Itô's formula. In our case Itô's formula allows to see the connection between the solutions of one special type of stochastic differential equation and the solutions of the system of partial differential equations.

More precisely, the formulation of Itô's formula (for simplicity, one dimensional) is the following:

Theorem 1.1.3. (Itô's formula) Let $F(x, t)$ be twice continuously differentiable in $x$, and continuously differentiable in $t$ and $X_{t}$ be an Itô process. Then $F\left(X_{t}, t\right)$ is also an Itô process and it holds

$$
\begin{aligned}
F\left(X_{t}, t\right)=F( & \left.X_{0}, 0\right) \\
+\int_{0}^{t}\left[\frac{\partial F}{\partial t}\left(X_{s}, s\right)+\right. & \left.\frac{\partial F}{\partial x}\left(X_{s}, s\right) b_{s}+\frac{1}{2} \frac{\partial^{2} F}{\partial x^{2}}\left(X_{s}, s\right) \sigma_{s}^{2}\right] d s \\
& +\int_{0}^{t} \frac{\partial F}{\partial x}\left(X_{s}, s\right) \sigma_{s} d W_{s}, \quad \forall t \in[0, T], \mathbb{P}-\text { a.s. }
\end{aligned}
$$

A proof of the Itô's formula can be found in [12]. 


\section{PRELIMINARIES}

\section{Weak convergence}

In this part we consider the convergence of sequences of processes valued in the space of càdlàg functions $\mathbb{D}\left([0, T] ; \mathbb{R}^{k}\right)$ in „weak" sense which means in distribution.

Let $(U, \pi)$ be a Polish space (that is a complete separable metric space) with its Borel $\sigma$-field $\mathcal{B}(U)$. Denote by $\mathcal{P}(U)$ the set of all probability measures on the measurable space $(U, \mathcal{B}(U))$. A sequence $\left(\mathbb{P}_{n}\right)_{n \in \mathbb{N}} \subseteq \mathcal{P}(U)$ is said to be weakly convergent to $\mathbb{P} \in \mathcal{P}(U)$ if for any bounded continuous function $f: U \rightarrow \mathbb{R}$ it holds that

$$
\lim _{n \rightarrow \infty} \int_{U} f(u) \mathbb{P}_{n}(d u)=\int_{U} f(u) \mathbb{P}(d u) .
$$

Weak convergence of random variables is defined from the weak convergence of probability measures. Namely, considering a sequence $X,\left(X_{n}\right)_{n \in \mathbb{N}}$ of $U$-valued random variables, defined on the spaces $(\Omega, \mathcal{F}, \mathbb{P})$, respectively, $\left(\Omega_{n}, \mathcal{F}_{n}, \mathbb{P}_{n}\right)$, we say that $\left(X_{n}\right)_{n \in \mathbb{N}}$ converges weakly to $X$ if $\left(\mathbb{P}_{X_{n}}\right)_{n \in \mathbb{N}}$ converges weakly to $\mathbb{P}_{X}$ in $\mathcal{P}(U)$.

A set $A \subseteq \mathcal{P}(U)$ is said to be relatively compact if any sequence $\left(\mathbb{P}_{n}\right)_{n \in \mathbb{N}} \subseteq A$ contains a weakly convergent subsequence; $A$ is tight if for any $\varepsilon>0$ there exists a compact set $K \subseteq U$ so that

$$
\inf _{\mathbb{P} \in A} \mathbb{P}[K] \geq 1-\varepsilon
$$

The famous Prokhorov's theorem (see [28]) states that the sequence $\left(\mathbb{P}_{n}\right)_{n \in \mathbb{N}}$ is relatively compact in $\mathcal{P}(U)$ if and only if the sequence $\left(\mathbb{P}_{n}\right)_{n \in \mathbb{N}}$ is tight. Recall, that we need here to be $(U, \pi)$ a Polish space.

Hence, to define weak convergence of a family of measures

$$
\left(\mathbb{P}_{n}\right)_{n \in \mathbb{N}} \subseteq \mathcal{P}\left(\mathbb{D}\left([0, T] ; \mathbb{R}^{k}\right)\right)
$$

we have to define a metric $\delta$ on $\mathbb{D}\left([0, T] ; \mathbb{R}^{k}\right)$ so that $\left(\mathbb{D}\left([0, T] ; \mathbb{R}^{k}\right), \delta\right)$ is a Polish space.

Let $\Lambda$ be the space of all continuous functions $\lambda:[0, T] \rightarrow[0, T]$ such that they are strictly increasing with $\lambda(0)=0$. For $\alpha, \beta \in \mathbb{D}\left([0, T] ; \mathbb{R}^{k}\right)$ we define a distance $\delta$ on $\mathbb{D}\left([0, T] ; \mathbb{R}^{k}\right)$ as

$$
\delta(\alpha, \beta):=\inf _{\lambda \in \Lambda} \max \left\{\sup _{t \in[0, T]}\|\lambda(t)-t\|_{2}, \sup _{t \in[0, T]}\|\alpha(t)-\beta(\lambda(t))\|_{2}\right\} .
$$

Using, for example, [5] or [14] it follows that $\left(\mathbb{D}\left([0, T] ; \mathbb{R}^{k}\right), \delta\right)$ is a Polish space. The topology on $\left(\mathbb{D}\left([0, T] ; \mathbb{R}^{k}\right)\right.$ induced by $\delta$ is called the Skorokhod topology. 


\subsection{From BSDEs to BSVIEs}

This section gives an introduction to the theory of terminal value problems of stochastic differential equations involving the Itô stochastic integral. In Subsection 1.2.1 we formulate the main well-posedness result for backward stochastic differential equations and show a connection between a family of those equations and backward stochastic Volterra integral equations. In Subsection 1.2.2 we show the well-posedness result for backward stochastic Volterra integral equations.

\subsubsection{BSDEs}

In this subsection we would like to introduced BSDEs in detail and state the well-posedness theorem for solutions of BSDEs.

\section{Existence and uniqueness results}

Let $W=\left(W_{t}\right)_{t \in[0, T]}$ be a standard $d$-dimensional Brownian motion defined on $(\Omega, \mathcal{F}, \mathbb{F}, \mathbb{P})$, where $\mathbb{F}=\left(\mathcal{F}_{t}\right)_{t \in[0, T]}$ is the natural filtration of $W$, and $T>0$ is a fixed terminal time. Note that we use these assumptions throughout this thesis if nothing changes.

Definition 1.2.1. For $\xi: \Omega \rightarrow \mathbb{R}^{k}$ and $h:[0, T] \times \mathbb{R}^{k} \times \mathbb{R}^{k \times d} \rightarrow \mathbb{R}^{k}$ we call a stochastic integral equation of Itô's type of the form

$$
Y_{t}=\xi-\int_{t}^{T} h\left(s, Y_{s}, Z_{s}\right) d s-\int_{t}^{T} Z_{s} d W_{s}, t \in[0, T]
$$

$a$ backward stochastic differential equation. The function $h$ is called the generator and the random variable $\xi$ the terminal condition.

Remark 1.2.2. In general the generator $h$ can also depend on $\omega \in \Omega$ in a nonanticipative way, but throughout this thesis we consider only generators which are independent on $\omega$.

Assume that in $(1.2)$ the terminal condition $\xi$ and generator $h$ satisfy the following conditions:

(B1) $\xi \in \mathbb{L}_{\mathfrak{F}_{T}}^{2}\left(\Omega ; \mathbb{R}^{k}\right)$.

(B2) $h$ is a $\mathcal{B}([0, T]) \otimes \mathcal{B}\left(\mathbb{R}^{k}\right) \otimes \mathcal{B}\left(\mathbb{R}^{k \times d}\right)$-measurable map fulfilling a Lipschitz condition, i.e. there exists a constant $L>0$ such that for all $t \in[0, T]$, $y_{i} \in \mathbb{R}^{k}, z_{i} \in \mathbb{R}^{k \times d}, i=1,2$, it holds that

$$
\left\|h\left(t, y_{1}, z_{1}\right)-h\left(t, y_{2}, z_{2}\right)\right\|_{2} \leq L\left(\left\|y_{1}-y_{2}\right\|_{2}+\left\|z_{1}-z_{2}\right\|_{2}\right) .
$$




\section{PRELIMINARIES}

Defining by

$$
\mathbb{H}^{2}[R, S]:=\mathbb{L}_{\mathbb{F}}^{2}\left(C([R, S]) ; \mathbb{R}^{k}\right) \times L_{\mathbb{F}}^{2}\left(R, S ; \mathbb{R}^{k \times d}\right),
$$

we formulate the following existence and uniqueness result of the solutions $(Y, Z)$.

Theorem 1.2.3. Let (B1) and (B2) hold. Then there exists a unique pair $(Y, Z) \in \mathbb{H}^{2}[0, T]$ which solves the BSDE (1.2).

Proof. The proof is based on the martingale representation theorem 1.1.1 for the Brownian motion (to obtain a unique $\mathbb{F}$-adapted solution $(Y, Z)$ in the linear case) and the contraction mapping theorem (to switch over from linear BSDEs to the unique solution $(Y, Z)$ in the nonlinear case). For details see, for example, [9].

Note that the condition of $\mathbb{F}$-adaptedness on $(Y, Z)$ in Theorem 1.2 .3 naturally appears as solutions of Itô stochastic differential equations are assumed to be $\mathbb{F}$-adapted and the process $Z$ is constructed by a martingale representation, see also [9, 32] for some counter-examples.

\section{Families of BSDEs}

In this part we want to show a connection between backward stochastic Volterra integral equations and a family of BSDEs parameterized in time. This is a useful result for proving the well-posedness Theorem 1.2.9 for backward stochastic Volterra integral equations in general form.

Denoting the sets

$$
\begin{aligned}
& \Delta[R, S]:=\left\{(t, s) \in[R, S]^{2}: \quad R \leq s \leq t \leq S\right\}, \\
& \Delta^{c}[R, S]:=\left\{(t, s) \in[R, S]^{2}: \quad R \leq t<s \leq S\right\},
\end{aligned}
$$

we introduce the following stochastic equation:

Definition 1.2.4. For $f: C\left([R, T] ; \mathbb{R}^{d}\right) \times[R, T] \rightarrow \mathbb{R}^{k}$ and $h:[S, T] \times \mathbb{R}^{k \times d} \times$ $[R, T] \rightarrow \mathbb{R}^{k}$ we say that a family of stochastic integral equations of Itô's type of the form

$$
\lambda_{t, r}=f(W ; t)-\int_{r}^{T} h\left(s, \mu_{t, s} ; t\right) d s-\int_{r}^{T} \mu_{t, s} d W_{s}, r \in[S, T], t \in[R, T],
$$

is a family of BSDEs on $[S, T]$ parameterized by $t \in[R, T]$, or a family of stochastic Fredholm integral equations (SFIEs, for short) on $[R, T]$ parameterized by $r \in[S, T]$. 


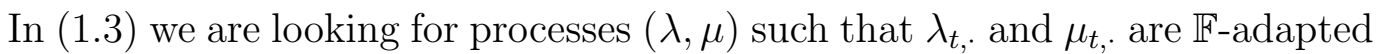
for almost all $t \in[R, T]$ under the following assumptions for the free term $f$ and generator $h$ :

(F1) $f \in L_{\mathcal{F}_{T}}^{2}\left(C\left([R, T] ; \mathbb{R}^{d}\right) \times[R, T] ; \mathbb{R}^{k}\right)$.

(F2) $h$ is a $\mathcal{B}([S, T]) \otimes \mathcal{B}\left(\mathbb{R}^{k \times d}\right) \otimes \mathcal{B}([R, T])$-measurable map fulfilling a Lipschitz condition, i.e. there exists a constant $L>0$ such that for all $(t, s) \in$ $[R, T] \times[S, T], z_{i} \in \mathbb{R}^{k \times d}, i=1,2$, it holds that

$$
\left\|h\left(s, z_{1} ; t\right)-h\left(s, z_{2} ; t\right)\right\|_{2} \leq L\left(\left\|z_{1}-z_{2}\right\|_{2}\right) .
$$

Using the previous assumptions we state the well-posedness result for SFIEs (1.3):

Theorem 1.2.5. Let (F1) and (F2) hold. Then there exists a unique adapted solution $\left(\lambda_{t,}, \mu_{t, .}\right) \in \mathbb{H}^{2}[S, T]$ for almost all $t \in[R, T]$ which solves the SFIE (1.3).

Proof. The proof can be found in [35], Lemma 3.3.

Remark 1.2.6. We want to mention one interesting thing about the equation (1.3). Namely, for $R=S$ we denote

$$
\left\{\begin{array}{l}
Y_{t}:=\lambda_{t, t}, \quad t \in[S, T] \\
Z_{t, s}:=\mu_{t, s}, \quad(t, s) \in \Delta^{c}[S, T] .
\end{array}\right.
$$

Thus, one obtains from (1.3) the equation

$$
Y_{t}=f(W ; t)-\int_{t}^{T} h\left(s, Z_{t, s} ; t\right) d s-\int_{t}^{T} Z_{t, s} d W_{s}, t \in[S, T],
$$

which is a special case of a BSVIE (1.6) (see Definition 1.2.7). Defining $Z_{t, s}$ for $(t, s) \in \Delta[S, T]$ by (1.7) below we get the well-posedness result for (1.5) (see [35], Corollary 3.6).

\subsubsection{BSVIEs}

Similar to the previous subsection, the aim of this part is to present BSVIEs in the general form and to state the well-posedness theorem for solutions of such equations.

\section{Well-posedness of BSVIEs}

In what follows, we define a backward stochastic Volterra integral equation: 


\section{PRELIMINARIES}

Definition 1.2.7. For $f: C\left([0, T] ; \mathbb{R}^{d}\right) \times[0, T] \rightarrow \mathbb{R}^{k}$ and $h:[0, T] \times \mathbb{R}^{k} \times \mathbb{R}^{k \times d} \times$ $\mathbb{R}^{k \times d} \times[0, T] \rightarrow \mathbb{R}^{k}$ we call a stochastic integral equation of Itô's type of the form

$$
Y_{t}=f(W ; t)-\int_{t}^{T} h\left(s, Y_{s}, Z_{t, s}, Z_{s, t} ; t\right) d s-\int_{t}^{T} Z_{t, s} d W_{s}, t \in[0, T],
$$

$a$ backward stochastic Volterra integral equation. The process $f$ is called the free term and the function $h$ the generator.

Note, that equation (1.6) cannot be reduced to a BSDE in general because the free term $f$ or the generator $h$ may depend on $t$.

Equation (1.6) has in general infinitely many adapted solutions. To achieve uniqueness, as pointed out in [35], we consider only adapted M-solutions. Such solutions of the BSVIE fulfill a martingale representation property, which determines the process $Z_{t, s}$ for $t \geq s$. Namely, denoting by

$$
\mathcal{H}^{2}[R, S]:=L_{\mathbb{F}}^{2}\left(R, S ; \mathbb{R}^{k}\right) \times L^{2}\left(R, S ; L_{\mathbb{F}}^{2}\left(R, S ; \mathbb{R}^{k \times d}\right)\right),
$$

we formulate the following definition:

Definition 1.2.8. Let $S \in[0, T)$. A pair $(Y, Z) \in \mathcal{H}^{2}[S, T]$ is called an adapted M-solution of the BSVIE (1.6) on $[S, T]$ if (1.6) holds in the usual Itô's sense for almost all $t \in[S, T]$ and $Y_{t}$ has the following martingale representation:

$$
Y_{t}=\mathbb{E}\left[Y_{t} \mid \mathcal{F}_{S}\right]+\int_{S}^{t} Z_{t, s} d W_{s}, \quad \text { a.e. } t \in[S, T] .
$$

To obtain the well-posedness for the BSVIE (1.6) we introduce the assumptions for the free term $f$ and generator $h$ :

$\left(\mathbf{V} 1^{\prime}\right) f \in L_{\mathcal{F}_{T}}^{2}\left(C\left([0, T] ; \mathbb{R}^{d}\right) \times[0, T] ; \mathbb{R}^{k}\right)$.

$\left(\mathbf{V 2}^{\prime}\right) h$ is a $\mathcal{B}([0, T]) \otimes \mathcal{B}\left(\mathbb{R}^{k}\right) \otimes \mathcal{B}\left(\mathbb{R}^{k \times d}\right) \otimes \mathcal{B}\left(\mathbb{R}^{k \times d}\right) \otimes \mathcal{B}([0, T])$-measurable map fulfilling a Lipschitz condition, i.e. there exists a constant $L^{\prime}>0$ such that for all $(t, s) \in \Delta^{c}[0, T], y_{i} \in \mathbb{R}^{k}, z_{i}, \bar{z}_{i} \in \mathbb{R}^{k \times d}, i=1,2$, it holds that

$$
\begin{aligned}
& \| h\left(s, y_{1}, z_{1}, \bar{z}_{1} ; t\right)-h\left(s, y_{2}, z_{2}, \bar{z}_{2} ; t\right) \|_{2} \\
& \leq L^{\prime}\left(\left\|y_{1}-y_{2}\right\|_{2}+\left\|z_{1}-z_{2}\right\|_{2}+\left\|\bar{z}_{1}-\bar{z}_{2}\right\|_{2}\right) .
\end{aligned}
$$

Theorem 1.2.9. Let $\left(\mathbf{V} 1^{\prime}\right)$ and $\left(\mathbf{V} \mathbf{2}^{\prime}\right)$ hold. Then there exists a unique adapted $M$-solution $(Y, Z) \in \mathcal{H}^{2}[0, T]$ which solves the BSVIE (1.6). 
Sketch of the proof. We give here the main four steps of the proof. In step (i) the process $Z_{t, s}$ is constructed on the subset $A_{i} \subset[0, T]^{2}, i=1, \ldots, 4$, defined below (see Figure 1.1). For details of the proof see [35].

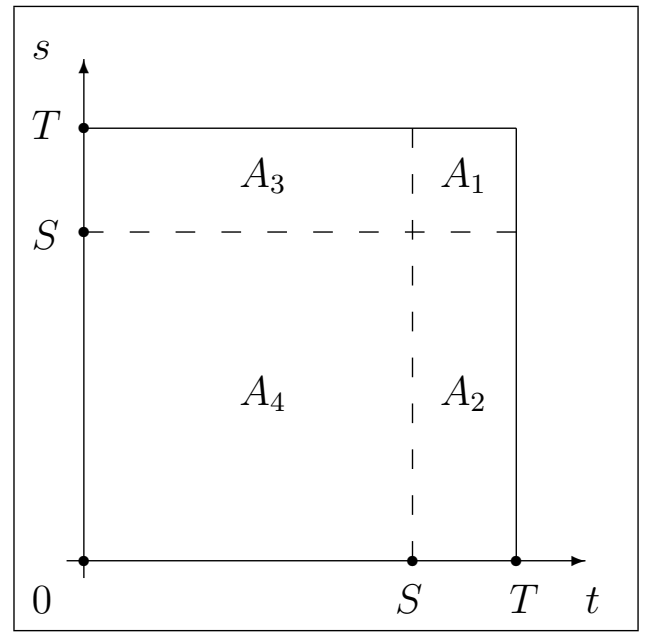

Figure 1.1: The sets $A_{1}, \ldots, A_{4}$ in $[0, T]^{2}$ for the values of the process $Z$.

(1) Note that for each pair of processes $(y, z)$, taken from an appropriate space (see [35]), the BSVIE

$$
Y_{t}=f(W ; t)-\int_{t}^{T} h\left(s, y_{s}, Z_{t, s}, z_{s, t} ; t\right) d s-\int_{t}^{T} Z_{t, s} d W_{s}, t \in[S, T],
$$

has a unique $\mathbb{F}$-adapted M-solution $(Y, Z)$ due to Remark 1.2 .6 for any $S \in$ $[0, T)$. If $T-S$ is small enough, one can show that the map $(y, z) \mapsto(Y, Z)$ is a contraction in $\mathcal{H}^{2}[T-S, T]$. Hence, by the contraction mapping theorem, we find a unique $\mathbb{F}$-adapted M-solution

$$
\left\{\begin{array}{l}
Y_{t}, \quad t \in[S, T] \\
Z_{t, s},(t, s) \in\left\{(t, s) \in[0, T]^{2}: t, s \geq S\right\}=: A_{1}
\end{array}\right.
$$

of Equation (1.6) on $[S, T]$.

(2) The martingale representation theorem, see Theorem 1.1.1, determines

$$
Z_{t, s} \text { for }(t, s) \in\left\{(t, s) \in[0, T]^{2}: t \geq S, s \in[0, S]\right\}=: A_{2}
$$

in $L^{2}\left(S, T ; L_{\mathbb{F}}^{2}\left(0, S ; \mathbb{R}^{k \times d}\right)\right)$. 


\section{PRELIMINARIES}

(3) Using Theorem 1.2.5, the SFIE of the form

$$
Y_{t}^{S}=f(W ; t)-\int_{S}^{T} h^{S}\left(s, Z_{t, s} ; t\right) d s-\int_{S}^{T} Z_{t, s} d W_{s}, t \in[0, S],
$$

where $h^{S}(s, z ; t):=h\left(s, Y_{s}, z, Z_{s, t} ; t\right),(s, z, t) \in[S, T] \times \mathbb{R}^{k \times d} \times[0, S]$, admits a unique adapted solution

$$
\left(Y^{S}, Z\right) \in L_{\mathcal{F}_{S}}^{2}\left(0, S ; \mathbb{R}^{k}\right) \times L^{2}\left(0, S ; L_{\mathbb{F}}^{2}\left(S, T ; \mathbb{R}^{k \times d}\right)\right) .
$$

Note that the values $Y_{s}$ and $Z_{s, t}$ were already determined in the steps (1) and (2). Thus, we have uniquely found

$$
Z_{t, s} \text { for }(t, s) \in\left\{(t, s) \in[0, T]^{2}: t \in[0, S], s \geq S\right\}=: A_{3} .
$$

(4) For $R \in[0, S)$ small enough one obtains by induction the solvability of the equation

$$
Y_{t}=Y_{t}^{S}-\int_{t}^{S} h\left(s, Y_{s}, Z_{t, s}, Z_{s, t} ; t\right) d s-\int_{t}^{S} Z_{t, s} d W_{s}, t \in[R, S],
$$

where $Y^{S}$ is the solution from (1.8).

Repeating this procedure we end with the solution

$$
\left\{\begin{array}{l}
Y_{t}, \quad t \in[0, S] \\
Z_{t, s},(t, s) \in\left\{(t, s) \in[0, T]^{2}: t, s \in[0, S]\right\}=: A_{4} .
\end{array}\right.
$$

\subsection{Main Result of this Thesis}

We consider a special case of (1.6), namely, the equation

$$
Y_{t}=f(W ; t)-\int_{t}^{T} h\left(s, Y_{s}\right) d s-\int_{t}^{T} Z_{t, s} d W_{s}, t \in[0, T]
$$

which will be used later throughout this thesis. To obtain the well-posedness for the BSVIE (1.9) we introduce the assumptions for the free term $f$ and generator $h$ : 
(V1) $f \in L_{\mathcal{F}_{T}}^{2}\left(C\left([0, T] ; \mathbb{R}^{d}\right) \times[0, T] ; \mathbb{R}^{k}\right)$

(V2) $h$ is a $\mathcal{B}([0, T]) \otimes \mathcal{B}\left(\mathbb{R}^{k}\right)$-measurable map fulfilling a Lipschitz condition, i.e. there exists a constant $L>0$ such that for all $s \in[0, T], y_{i} \in \mathbb{R}^{k}, i=1,2$, it holds that

$$
\left\|h\left(s, y_{1}\right)-h\left(s, y_{2}\right)\right\|_{2} \leq L\left(\left\|y_{1}-y_{2}\right\|_{2}\right)
$$

We construct a numerical method to approximate the M-solution of (1.9). In [18] a numerical method is presented to approximate usual BSDEs, where the solutions are related with solutions of quasilinear PDEs of parabolic type. Our aim is to generalize this method to BSVIEs.

The discretization of the BSVIEs (1.9) is based upon replacing the $d$-dimensional Brownian motion $W_{s}$ by a simple symmetric random walk $W_{s}^{(n)}$ (see Theorem 1.1.2). Note that from Skorokhod's representation theorem we can assume that $W^{(n)}, W$ are defined on the same space $\Omega$ and $W^{(n)}$ converges a.s. uniformly on $[0, T]$ to $W$.

We introduce the following discretized version of BSVIE (1.9):

Definition 1.3.1. For $f: C\left([0, T] ; \mathbb{R}^{d}\right) \times[0, T] \rightarrow \mathbb{R}^{k}, h:[0, T] \times \mathbb{R}^{k} \rightarrow \mathbb{R}^{k}$ and the discretization $0=t_{0}<t_{1}<\ldots<t_{n}=T$ of the time interval $[0, T]$ with time-step $\frac{T}{n}\left(t_{i}:=\frac{i T}{n}, i=0, \ldots, n, n \in \mathbb{N}\right)$, we call an equation from (1.9) of the form

$$
Y_{t_{i}}^{(n)}=f\left(W^{(n)} ; t_{i}\right)-\sum_{j=i}^{n-1} h\left(t_{j}, Y_{t_{j}}^{(n)}\right) \Delta t_{j+1}-\sum_{j=i}^{n-1} Z_{t_{i}, t_{j}}^{(n)} \Delta W_{t_{j+1}}^{(n)}
$$

a discrete backward stochastic Volterra integral equation (DBSVIE, for short), where

$$
Y_{t_{i}}^{(n)}:=\left(\begin{array}{c}
Y_{t_{i}}^{1,(n)} \\
\vdots \\
Y_{t_{i}}^{k,(n)}
\end{array}\right), \sum_{j=i}^{n-1} Z_{t_{i}, t_{j}}^{(n)} \Delta W_{t_{j+1}}^{(n)}:=\left(\begin{array}{c}
\sum_{J=1}^{d} \sum_{j=i}^{n-1} Z_{t_{i}, t_{j}}^{1 J(n)} \Delta W_{t_{j+1}}^{J,(n)} \\
\vdots \\
\sum_{J=1}^{d} \sum_{j=i}^{n-1} Z_{t_{i}, t_{j}}^{k J,(n)} \Delta W_{t_{j+1}}^{J,(n)}
\end{array}\right)
$$

with $\Delta t_{j+1}:=t_{j+1}-t_{j}=\frac{T}{n}$.

Define discrete spaces of the DBSVIE 1.10 for $i_{0}, i_{1} \in\{0, \ldots, n\}$ : 
$L_{\mathcal{F}_{t_{n}}^{(n)}}^{2}\left(C\left(\left[t_{i_{0}}, t_{i_{1}}\right] ; \mathbb{R}^{l}\right) \times\left[t_{i_{0}}, t_{i_{1}}\right] ; \mathbb{R}^{k}\right)$ the space of $\mathcal{B}\left(C\left(\left[t_{i_{0}}, t_{i_{1}}\right] ; \mathbb{R}^{l}\right)\right) \otimes \mathcal{B}\left(\left[t_{i_{0}}, t_{i_{1}}\right]\right)$ measurable processes $f: C\left(\left[t_{i_{0}}, t_{i_{1}}\right] ; \mathbb{R}^{l}\right) \times$ $\left[t_{i_{0}}, t_{i_{1}}\right] \rightarrow \mathbb{R}^{k}$ such that $f\left(W^{(n)}(\cdot) ; t_{i}\right)$ is $\mathcal{F}_{t_{n}}^{(n)}$-measurable for all $i=i_{0}, \ldots, i_{1}$ and it holds $\mathbb{E}\left[\frac{T}{n} \sum_{i=i_{0}}^{i_{1}-1}\left\|f\left(W^{(n)}(\cdot) ; t_{i}\right)\right\|_{2}^{2}\right]<\infty$ with $\mathcal{B}\left(C\left(\left[t_{i_{0}}, t_{i_{1}}\right] ; \mathbb{R}^{l}\right)\right)$ the Borel $\sigma$-field induced by the topology, generated by the $\|\cdot\|_{\infty}$-norm on $C\left(\left[t_{i_{0}}, t_{i_{1}}\right] ; \mathbb{R}^{l}\right)$.

$L_{\mathbb{F}(n)}^{2}\left(t_{i_{0}}, t_{i_{1}} ; \mathbb{R}^{k}\right)$

the space of processes $Y^{(n)}$ : $\Omega \times\left\{t_{i_{0}}, \ldots, t_{i_{1}}\right\} \quad \rightarrow \quad \mathbb{R}^{k}$ such that $Y^{(n)}$ is $\mathbb{F}^{(n)}$-adapted and it holds $\mathbb{E}\left[\frac{T}{n} \sum_{i=i_{0}}^{i_{1}-1}\left\|Y^{(n)}\left(\cdot, t_{i}\right)\right\|_{2}^{2}\right]<\infty$.

We define $\left\|Y^{(n)}\right\|_{L_{\mathbb{F}^{(n)}}^{2}\left(t_{i_{0}}, t_{i_{1}} ; \mathbb{R}^{k}\right)} \quad:=$ $\left(\mathbb{E}\left[\frac{T}{n} \sum_{i=i_{0}}^{i_{1}-1}\left\|Y^{(n)}\left(\cdot, t_{i}\right)\right\|_{2}^{2}\right]\right)^{\frac{1}{2}}$.

$L^{2,(n)}\left(t_{i_{0}}, t_{i_{1}} ; \mathbb{R}^{k \times d}\right)$

the space of all processes $Z^{(n)}$ : $\Omega \times\left\{t_{i_{0}}, \ldots, t_{i_{1}}\right\}^{2} \rightarrow \mathbb{R}^{k \times d}$ such that $Z^{(n)}\left(\omega, t_{i}, t_{j}\right) \quad$ is $\quad\left(\mathcal{F}_{t_{j}}^{(n)}\right)_{j=i_{0}, \ldots, i_{1}-}$ adapted for all $t_{i}, i=i_{0}, \ldots, i_{1}$, and $\mathbb{E}\left[\left(\frac{T}{n}\right)^{2} \sum_{i=i_{0}}^{i_{1}-1} \sum_{j=i_{0}}^{i_{1}-1}\left\|Z^{(n)}\left(\cdot, t_{i}, t_{j}\right)\right\|_{2}^{2}\right]<\infty$. We define $\left\|Z^{(n)}\right\|_{L^{2,(n)}\left(t_{i_{0}}, t_{i_{1}} ; \mathbb{R}^{k \times d}\right)} \quad:=$ $\left(\mathbb{E}\left[\left(\frac{T}{n}\right)^{2} \sum_{i=i_{0}}^{2} \sum_{j=i_{0}}^{i_{1}-1}\left\|Z^{(n)}\left(\cdot, t_{i}, t_{j}\right)\right\|_{2}^{2}\right]\right)^{\frac{1}{2}}$.

$L_{\mathbb{F}^{(n)}}^{2, \infty}\left(t_{i_{0}}, t_{i_{1}} ; \mathbb{R}^{k}\right)$

the space of processes $Y^{(n)}$ : $\Omega \times\left\{t_{i_{0}}, \ldots, t_{i_{1}}\right\} \rightarrow \mathbb{R}^{k}$ such that $Y^{(n)}$ is $\mathbb{F}^{(n)}$-adapted and it holds $\mathbb{E}\left[\sup _{i \in\left\{i_{0}, \ldots, i_{1}\right\}}\left\|Y^{(n)}\left(\cdot, t_{i}\right)\right\|_{2}^{2}\right]<\infty$.

We define $\quad\left\|Y^{(n)}\right\|_{L_{\mathbb{F}}^{2, \infty}\left(t_{i_{0}}, t_{i_{1}} ; \mathbb{R}^{k}\right)} \quad:=$ $\left(\mathbb{E}\left[\sup _{i \in\left\{i_{0}, \ldots, i_{1}\right\}}\left\|Y^{(n)}\left(\cdot, t_{i}\right)\right\|_{2}^{2}\right]\right)^{\frac{1}{2}}$. 
Analogously to (1.6) also equation 11.10 can be solved uniquely by a pair $\left(Y^{(n)}, Z^{(n)}\right)$ if the pair $\left(Y^{(n)}, Z^{(n)}\right)$ fulfills a "discrete" martingale representation property. Namely, denoting by

$$
\mathcal{H}^{2,(n)}\left[t_{i_{0}}, t_{i_{1}}\right]:=L_{\mathbb{F}^{(n)}}^{2}\left(t_{i_{0}}, t_{i_{1}} ; \mathbb{R}^{k}\right) \times L^{2,(n)}\left(t_{i_{0}}, t_{i_{1}} ; \mathbb{R}^{k \times d}\right)
$$

with the norm

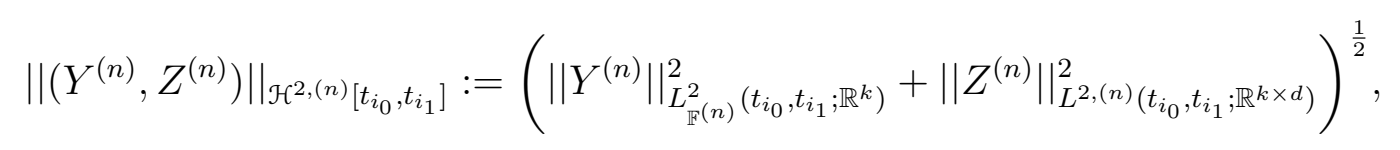

we formulate the following definition:

Definition 1.3.2. Let $\mathcal{S} \in\{0,1, \ldots, i-1\}$. A pair $\left(Y^{(n)}, Z^{(n)}\right) \in \mathcal{H}^{2,(n)}[0, T]$ is called an adapted M-solution of the DBSVIE (1.10), if $\left(Y^{(n)}, Z^{(n)}\right)$ solves 1.10 and $Y_{t_{i}}^{(n)}$ has the following discrete martingale representation:

$$
Y_{t_{i}}^{(n)}=\mathbb{E}\left[Y_{t_{i}}^{(n)} \mid \mathcal{F}_{t_{\delta}}^{(n)}\right]+\sum_{j=\delta}^{i-1} Z_{t_{i}, t_{j}}^{(n)} \Delta W_{t_{j+1}}^{(n)}
$$

Lemma 1.3.3. Suppose that the DBSVIE has an adapted $M$-solution $\left(Y^{(n)}, Z^{(n)}\right)$. Then $Z^{(n)}$ can be expressed in forms of $Y^{(n)}$ as follows: For $\mathcal{S}=0,1, \ldots, i-1$ it holds

$$
Z_{t_{i}, t_{\mathcal{S}}}^{(n)}= \begin{cases}\frac{1}{\Delta t_{\mathcal{S}+1}} \mathbb{E}\left[Y_{t_{i}}^{(n)}\left(\Delta W_{t_{\mathcal{S}+1}}^{(n)}\right)^{*} \mid \mathcal{F}_{t_{\mathcal{S}}}^{(n)}\right] & , \quad \mathcal{S}<i \\ \frac{1}{\Delta t_{\mathcal{S}+1}} \mathbb{E}\left[\left(f\left(W^{(n)} ; t_{i}\right)-\sum_{j=i}^{n-1} h\left(t_{j}, Y_{t_{j}}^{(n)}\right) \Delta t_{j+1}\right)\left(\Delta W_{t_{\mathcal{S}+1}}^{(n)}\right)^{*} \mid \mathcal{F}_{t_{\mathcal{S}}}^{(n)}\right], & \mathcal{S} \geq i\end{cases}
$$

The proof of this lemma is given in Chapter 2, Section 2.1.

To obtain the well-posedness for the discrete BSVIE 1.10 we introduce the assumptions for the free term $f$ and generator $h$ :

(D1) $f \in L_{\mathcal{F}_{t_{n}}^{(n)}}^{2}\left(C\left([0, T] ; \mathbb{R}^{d}\right) \times[0, T] ; \mathbb{R}^{k}\right)$.

(D2) $h$ is a $\mathcal{B}([0, T]) \otimes \mathcal{B}\left(\mathbb{R}^{k}\right)$-measurable map fulfilling a Lipschitz condition, i.e. there exists a constant $L>0$ such that for all $s \in[0, T], y_{i} \in \mathbb{R}^{k}, i=1,2$, it holds that

$$
\left\|h\left(s, y_{1}\right)-h\left(s, y_{2}\right)\right\|_{2} \leq L\left(\left\|y_{1}-y_{2}\right\|_{2}\right)
$$




\section{PRELIMINARIES}

Theorem 1.3.4. Let (D1) and (D2) hold. Then for n large enough (depending on the Lipschitz constant $L)$ there exists a unique adapted $M$-solution $\left(Y^{(n)}, Z^{(n)}\right) \in$ $\mathcal{H}^{2,(n)}[0, T]$ which solves the discrete BSVIE (1.10).

A proof of this theorem can be found in Section 2.2 .

The main result of this thesis is to show the weak convergence of the sequence $\left(Y^{(n)}\right)_{n \in \mathbb{N}}$ to $Y$. For this purpose we need the following assumptions:

(V3) The free term $f$ is a Lipschitz function, i.e. there exists a constant $K>0$ such that for all $g_{i} \in C\left([0, T] ; \mathbb{R}^{d}\right)$ and $t_{i} \in[0, T], i=1,2$, it holds that

$$
\left\|f\left(g_{1} ; t_{1}\right)-f\left(g_{2} ; t_{2}\right)\right\|_{2} \leq K\left(\left\|g_{1}-g_{2}\right\|_{\infty}+\left|t_{1}-t_{2}\right|\right) .
$$

(V4) The free term $f$ and the generator $h$ are functions bounded by a constant $D>0$, and $h$ is continuous.

Remark 1.3.5. In fact, we do not have to require that $h$ is bounded, since one can show with analogous arguments as in Lemma 3.1 from [18] that the processes $Y$ and $Y^{(n)}$ are bounded under the assumptions (V1)-(V3) with a bounded free term $f$.

Theorem 1.3.6. (Main Convergence Theorem) Assume that in the BSVIE (1.9) the assumptions (V1)-(V4) are fulfilled. Denote by $\left(Y^{(n)}, Z^{(n)}\right)_{n \in \mathbb{N}}$ the sequence of discrete $M$-solutions from (1.10) and by $(Y, Z)$ the adapted M-solution of (1.9). Then the sequence $\left(Y^{(n)}\right)_{n \in \mathbb{N}}$ converges weakly in the Skorokhod topology to $Y$, if it is piecewise constant interpolated between the points $Y_{t_{i}}^{(n)}$ and $Y_{t_{i+1}}^{(n)}$ for all $i=0, \ldots, n-1$.

Note that there exists a unique adapted M-solution $(Y, Z)$ of $(1.9)$ as conditions $\left(\mathbf{V} \mathbf{1}^{\prime}\right)$ and $\left(\mathbf{V 2}^{\prime}\right)$ of Theorem 1.2.9 are fulfilled, if the conditions (V1) and (V2) hold.

The sequence of processes $\left(Y^{(n)}, Z^{(n)}\right)_{n \in \mathbb{N}}$ can be approximated by a sequence of processes $\left(\hat{Y}^{(n)}, \hat{Z}^{(n)}\right)_{n \in \mathbb{N}}$ given by explicit equations. To understand this, remark that $Y_{t_{i}}^{(n)}$ from 1.10 can be written as

$$
\begin{aligned}
Y_{t_{i}}^{(n)}=Y_{t_{i+1}}^{(n)}+f\left(W^{(n)} ; t_{i}\right)- & f\left(W^{(n)} ; t_{i+1}\right)-h\left(t_{i}, Y_{t_{i}}^{(n)}\right) \Delta t_{i+1} \\
& +\sum_{j=i+1}^{n-1} Z_{t_{i+1}, t_{j}}^{(n)} \Delta W_{t_{j+1}}^{(n)}-\sum_{j=i}^{n-1} Z_{t_{i}, t_{j}}^{(n)} \Delta W_{t_{j+1}}^{(n)} .
\end{aligned}
$$

Taking the conditional expectation of $Y_{t_{i}}^{(n)}$ given $\mathcal{F}_{t_{i}}^{(n)}$ one obtains

$$
Y_{t_{i}}^{(n)}=\mathbb{E}\left[Y_{t_{i+1}}^{(n)}+f\left(W^{(n)} ; t_{i}\right)-f\left(W^{(n)} ; t_{i+1}\right) \mid \mathcal{F}_{t_{i}}^{(n)}\right]-h\left(t_{i}, Y_{t_{i}}^{(n)}\right) \Delta t_{i+1}
$$


In addition, denoting by

$$
X_{i}^{0}:=\mathbb{E}\left[Y_{t_{i+1}}^{(n)}+f\left(W^{(n)} ; t_{i}\right)-f\left(W^{(n)} ; t_{i+1}\right) \mid \mathcal{F}_{t_{i}}^{(n)}\right]
$$

the map $\Theta_{i}^{(n)}: \mathbb{L}_{\mathcal{F}_{t_{i}}^{(n)}}^{2}\left(\Omega ; \mathbb{R}^{k}\right) \rightarrow \mathbb{L}_{\mathcal{F}_{t_{i}}^{(n)}}^{2}\left(\Omega ; \mathbb{R}^{k}\right)$ which maps

$$
V \mapsto X_{i}^{0}-h\left(t_{i}, V\right) \Delta t_{i+1}
$$

is a contraction for large $n$ with fixed-point $Y_{t_{i}}^{(n)}$, since taking two different random variables $V$ and $\tilde{V}$ it holds

$\left\|\Theta_{i}^{(n)}(V)-\Theta_{i}^{(n)}(\tilde{V})\right\|_{2} \leq\left\|h\left(t_{i}, V\right) \Delta t_{i+1}-h\left(t_{i}, \tilde{V}\right) \Delta t_{i+1}\right\|_{2} \leq \frac{L T}{n}\|V-\tilde{V}\|_{2} \quad \mathbb{P}$-a.s.

where $\frac{L T}{n}<1$ for large $n$. Furthermore,

$$
\begin{gathered}
\left\|Y_{t_{i}}^{(n)}-\left(X_{i}^{0}-h\left(t_{i}, X_{i}^{0}\right) \Delta t_{i+1}\right)\right\|_{2}=\left\|X_{i}^{0}-h\left(t_{i}, Y_{t_{i}}^{(n)}\right) \Delta t_{i+1}-X_{i}^{0}+h\left(t_{i}, X_{i}^{0}\right) \Delta t_{i+1}\right\|_{2} \\
\leq \frac{L T}{n}\left\|Y_{t_{i}}^{(n)}-X_{i}^{0}\right\|_{2} \leq \frac{D L T^{2}}{n^{2}} \mathbb{P} \text {-a.s. }
\end{gathered}
$$

This motivates us to propose the following explicit numerical scheme for the BSVIE (1.9):

$$
\begin{aligned}
& \hat{Y}_{t_{n}}^{(n)}=f\left(W^{(n)} ; t_{n}\right), \quad \hat{Z}_{t_{n}, t_{n}}^{(n)}=0, \\
& \hat{X}_{t_{i}}^{(n)}=\mathbb{E}\left[\hat{Y}_{t_{i+1}}^{(n)}+f\left(W^{(n)} ; t_{i}\right)-f\left(W^{(n)} ; t_{i+1}\right) \mid \mathcal{F}_{t_{i}}^{(n)}\right], \\
& \hat{Y}_{t_{i}}^{(n)}=\hat{X}_{t_{i}}^{(n)}-h\left(t_{i}, \hat{X}_{t_{i}}^{(n)}\right) \Delta t_{i+1}, \\
& \hat{Z}_{t_{i}, t_{\mathcal{S}}}^{(n)}= \begin{cases}\frac{1}{\Delta t_{\mathcal{S}+1}} \mathbb{E}\left[\hat{Y}_{t_{i}}^{(n)}\left(\Delta W_{t_{S+1}}^{(n)}\right)^{*} \mid \mathcal{F}_{t_{S}}^{(n)}\right] & , \mathcal{S}<i \\
\frac{1}{\Delta t_{\mathcal{S}+1}} \mathbb{E}\left[\left(f\left(W^{(n)} ; t_{i}\right)-\sum_{j=i}^{n-1} h\left(t_{j}, \hat{Y}_{t_{j}}^{(n)}\right) \Delta t_{j+1}\right)\left(\Delta W_{t_{\delta_{+}}}^{(n)}\right)^{*} \mid \mathcal{F}_{t_{\mathcal{S}}}^{(n)}\right], & \mathcal{S} \geq i\end{cases}
\end{aligned}
$$

With analogous arguments as in [18, we can estimate the error between $Y^{(n)}$ and $\hat{Y}^{(n)}$ as well as between $Z^{(n)}$ and $\hat{Z}^{(n)}$. Namely,

$$
\begin{aligned}
\left\|Y_{t_{i}}^{(n)}-\hat{Y}_{t_{i}}^{(n)}\right\|_{2}=\left\|\mathbb{E}\left[Y_{t_{i+1}}^{(n)}-\hat{Y}_{t_{i+1}}^{(n)} \mid \mathcal{F}_{t_{i}}^{(n)}\right]-\left(h\left(t_{i}, Y_{t_{i}}^{(n)}\right)-h\left(t_{i}, \hat{X}_{t_{i}}^{(n)}\right)\right) \Delta t_{i+1}\right\|_{2} \\
\leq\left\|\mathbb{E}\left[Y_{t_{i+1}}^{(n)}-\hat{Y}_{t_{i+1}}^{(n)} \mid \mathcal{F}_{t_{i}}^{(n)}\right]\right\|_{2}+\left\|h\left(t_{i}, Y_{t_{i}}^{(n)}\right)-h\left(t_{i}, \hat{Y}_{t_{i}}^{(n)}\right)\right\|_{2} \Delta t_{i+1} \\
+\left\|h\left(t_{i}, \hat{Y}_{t_{i}}^{(n)}\right)-h\left(t_{i}, \hat{X}_{t_{i}}^{(n)}\right)\right\|_{2} \Delta t_{i+1} \leq \frac{D\left(e^{2 L}-1\right)}{n} \quad \mathbb{P} \text {-a.s. }
\end{aligned}
$$




\section{PRELIMINARIES}

and

$\left\|Z_{t_{i}, t_{s}}^{(n)}-\hat{Z}_{t_{i}, t_{8}}^{(n)}\right\|_{2}$

$= \begin{cases}\frac{1}{\Delta t_{\mathcal{S}+1}}\left\|\mathbb{E}\left[\left(Y_{t_{i}}^{(n)}-\hat{Y}_{t_{i}}^{(n)}\right)\left(\Delta W_{t_{\mathcal{S}+1}}^{(n)}\right)^{*} \mid \mathcal{F}_{t_{S}}^{(n)}\right]\right\|_{2} & , \quad \mathcal{S}<i \\ \frac{1}{\Delta t_{\mathcal{S}+1}}\left\|\mathbb{E}\left[\left(\sum_{j=i}^{n-1}\left(h\left(t_{j}, \hat{Y}_{t_{j}}^{(n)}\right)-h\left(t_{j}, Y_{t_{j}}^{(n)}\right)\right) \Delta t_{j+1}\right)\left(\Delta W_{t_{\mathcal{S}+1}}^{(n)}\right)^{*} \mid \mathcal{F}_{t_{\mathcal{S}}}^{(n)}\right]\right\|_{2} & , \quad \mathcal{S} \geq i\end{cases}$

$\leq\left\{\begin{array}{ll}\frac{\frac{1}{\sqrt{T}} D\left(e^{2 L}-1\right)}{\sqrt{n}}, & \mathcal{S}<i \quad \\ \frac{\sqrt{T} L D\left(e^{2 L}-1\right)}{\sqrt{n}}, & \mathbb{S} \geq i\end{array} \quad\right.$ P-a.s.

From this immediately follows with Theorem 1.3.6 the next corollary:

Corollary 1.3.7. The assertion of Theorem 1.3.6 also holds true if we consider, instead of the sequence $\left(Y^{(n)}\right)_{n \in \mathbb{N}}$, the sequence $\left(Y^{(n)}\right)_{n \in \mathbb{N}}$.

The proof of Theorem 1.3.6 is given in Chapter 2 and divided into three parts in Sections 2.3, 2.4 and 2.5. Section 2.3 is devoted to the estimations for the difference between different solutions from BSVIEs (1.9) and (1.10), respectively. In Section 2.4 we show the tightness of the sequence $\left(Y^{(n)}\right)_{n \in \mathbb{N}}$ from $(1.10)$. In Section 2.5 we present the weak convergence of the solution of Equation (1.10) to the solution of Equation 1.9 for $n \rightarrow \infty$ under certain regularity conditions, if the free term depends not on the whole trajectory of the Brownian motion but only on finitely many points.

The rest of the thesis is structured in Chapter 3 with one numerical example illustrating the speed of convergence of our algorithm and Chapter 4 , in which we summarize all the results given in this thesis and formulate some open questions for further work. 


\section{Chapter 2}

\section{Proofs}

This chapter is a collection of proofs for the problems formulated in Section 1.3. In Section 2.1 we construct the implicit equations for the process $Z^{(n)}$. In Section 2.2 we show the existence and uniqueness for the solutions from the discrete BSVIE 1.10). The proofs in Sections 2.3, 2.4 and 2.5 help us to show the Main Convergence Theorem 1.3.6. As a result, in Section 2.6 we prove Theorem 1.3.6.

\subsection{Proof of Lemma 1.3 .3}

Proof of Lemma 1.3.3. In the case $\mathcal{S}<i$, multiplying Equation 1.11) by $\Delta W_{t_{\mathcal{S}+1}}^{(n)}$ and taking the conditional expectation of this expression given $\mathcal{F}_{t_{\delta}}^{(n)}$ one obtains

$$
\begin{aligned}
\mathbb{E}\left[\left(Y_{t_{i}}^{(n)}-\mathbb{E}\left[Y_{t_{i}}^{(n)} \mid \mathcal{F}_{t_{\mathcal{S}}}^{(n)}\right]\right)\left(\Delta W_{t_{S+1}}^{(n)}\right)^{*} \mid \mathcal{F}_{t_{\mathcal{S}}}^{(n)}\right] & \\
= & \sum_{j=\mathcal{S}}^{i-1} \mathbb{E}\left[\left(Z_{t_{i}, t_{j}}^{(n)} \Delta W_{t_{j+1}}^{(n)}\right)\left(\Delta W_{t_{S+1}}^{(n)}\right)^{*} \mid \mathcal{F}_{t_{\mathcal{S}}}^{(n)}\right] .
\end{aligned}
$$

On the other hand, using the martingale property of the random walk and the tower property of conditional expectation, it follows that

$$
\mathbb{E}\left[\left(Y_{t_{i}}^{(n)}-\mathbb{E}\left[Y_{t_{i}}^{(n)} \mid \mathcal{F}_{t_{\mathcal{S}}}^{(n)}\right]\right)\left(\Delta W_{t_{\mathcal{S}+1}}^{(n)}\right)^{*} \mid \mathcal{F}_{t_{\mathcal{S}}}^{(n)}\right]=\mathbb{E}\left[Y_{t_{i}}^{(n)}\left(\Delta W_{t_{\mathcal{S}+1}}^{(n)}\right)^{*} \mid \mathcal{F}_{t_{\mathcal{S}}}^{(n)}\right]
$$

and

$$
\begin{aligned}
\mathbb{E}\left[\left(Z_{t_{i}, t_{j}}^{(n)} \Delta W_{t_{j+1}}^{(n)}\right)\left(\Delta W_{t_{S+1}}^{(n)}\right)^{*} \mid \mathcal{F}_{t_{S}}^{(n)}\right] \\
\quad= \begin{cases}Z_{t_{i}, t_{s}}^{(n)} \Delta t_{\mathcal{S}+1} \\
\mathbb{E}\left[\mathbb{E}\left[\left(Z_{t_{i}, t_{j}}^{(n)} \Delta W_{t_{j+1}}^{(n)}\right)\left(\Delta W_{t_{\delta+1}}^{(n)}\right)^{*} \mid \mathcal{F}_{t_{j+1}}^{(n)}\right] \mid \mathcal{F}_{t_{\delta}}^{(n)}\right]=0, & , j=\mathcal{S}\end{cases}
\end{aligned}
$$




\section{PROOFS}

Hence,

$$
Z_{t_{i}, t_{S}}^{(n)}=\frac{1}{\Delta t_{\mathcal{S}+1}} \mathbb{E}\left[Y_{t_{i}}^{(n)}\left(\Delta W_{t_{S+1}}^{(n)}\right)^{*} \mid \mathcal{F}_{t_{S}}^{(n)}\right]
$$

In the same spirit, for the case $\mathcal{S} \geq i$ we multiply Equation 1.10 by $\Delta W_{t_{S+1}}^{(n)}$ and take the conditional expectation given $\mathcal{F}_{t_{S}}^{(n)}$. One obtains

$$
\begin{aligned}
\mathbb{E}\left[Y_{t_{i}}^{(n)}\left(\Delta W_{t_{S+1}}^{(n)}\right)^{*} \mid \mathcal{F}_{t_{S}}^{(n)}\right]= & 0 \\
=\mathbb{E}\left[\left(f\left(W^{(n)} ; t_{i}\right)-\sum_{j=i}^{n-1} h\left(t_{j}, Y_{t_{j}}^{(n)}\right) \Delta t_{j+1}\right)\left(\Delta W_{t_{S+1}}^{(n)}\right)^{*} \mid \mathcal{F}_{t_{S}}^{(n)}\right] & \\
& -\sum_{j=i}^{n-1} \mathbb{E}\left[\left(Z_{t_{i}, t_{j}}^{(n)} \Delta W_{t_{j+1}}^{(n)}\right)\left(\Delta W_{t_{S+1}}^{(n)}\right)^{*} \mid \mathcal{F}_{t_{S}}^{(n)}\right],
\end{aligned}
$$

where

$$
\begin{array}{r}
\mathbb{E}\left[\left(Z_{t_{i}, t_{j}}^{(n)} \Delta W_{t_{j+1}}^{(n)}\right)\left(\Delta W_{t_{\mathcal{S}+1}}^{(n)}\right)^{*} \mid \mathcal{F}_{t_{\mathcal{S}}}^{(n)}\right] \\
= \begin{cases}Z_{t_{i}, t_{\mathcal{S}}}^{(n)} \Delta t_{\mathcal{S}+1} & , j=\mathcal{S} \\
\left(Z_{t_{i}, t_{j}}^{(n)} \Delta W_{t_{j+1}}^{(n)}\right)\left(\mathbb{E}\left[\Delta W_{t_{\mathcal{S}+1}}^{(n)} \mid \mathcal{F}_{t_{\mathcal{S}}}^{(n)}\right]\right)^{*}=0 & , j<\mathcal{S} \\
\mathbb{E}\left[\mathbb{E}\left[\left(Z_{t_{i}, t_{j}}^{(n)} \Delta W_{t_{j+1}}^{(n)}\right)\left(\Delta W_{t_{\mathcal{S}+1}}^{(n)}\right)^{*} \mid \mathcal{F}_{t_{j+1}}^{(n)}\right] \mid \mathcal{F}_{t_{\mathcal{S}}}^{(n)}\right]=0 & , j>\mathcal{S}\end{cases}
\end{array}
$$

Thus,

$$
Z_{t_{i}, t_{\mathcal{S}}}^{(n)}=\frac{1}{\Delta t_{\mathcal{S}+1}} \mathbb{E}\left[\left(f\left(W^{(n)} ; t_{i}\right)-\sum_{j=i}^{n-1} h\left(t_{j}, Y_{t_{j}}^{(n)}\right) \Delta t_{j+1}\right)\left(\Delta W_{t_{\mathcal{S}+1}}^{(n)}\right)^{*} \mid \mathcal{F}_{t_{\mathcal{S}}}^{(n)}\right]
$$

\subsection{Well-posedness of Discrete BSVIEs}

The proof of Theorem 1.3.4 relies on the fact that the discrete version of a BSDE of the form

$$
Y_{t_{i}}^{(n)}=\xi\left(W^{(n)}\right)-\frac{T}{n} \sum_{j=i}^{n-1} h\left(t_{j}, Y_{t_{j}}^{(n)}\right)-\sum_{j=i}^{n-1} Z_{t_{j}}^{(n)} \Delta W_{t_{j+1}}^{(n)}
$$

can be solved uniquely: 
Lemma 2.2.1. Let

$$
\xi \in L_{\mathcal{F}_{t_{n}}^{(n)}}^{2}\left(C\left([0, T] ; \mathbb{R}^{d}\right) ; \mathbb{R}^{k}\right)
$$

and (D2) hold. Then for $n$ large enough (depending on the Lipschitz constant $L)$ there exists a unique $\mathbb{F}^{(n)}$-adapted solution $\left(Y_{t_{i}}^{(n)}, Z_{t_{i}}^{(n)}\right) \in \mathcal{H}^{2,(n)}$ which solves (2.1).

Proof. A proof can be found in [25].

Proof of Theorem 1.3.4. In the proof of this theorem we use similar ideas to construct the sequences on subsets of $\left\{t_{0}, \ldots, t_{n}\right\}^{2}\left(t_{0}=0, t_{n}=T\right)$ on every step $(i)$ (see Figure 2.1) as in Theorem 1.2.9 in the continuous case, $i=1, \ldots, 4$.

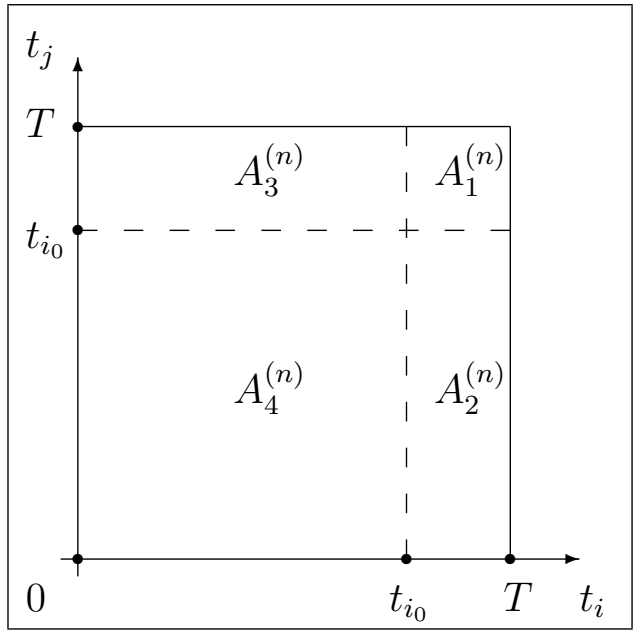

Figure 2.1: The sets $A_{1}^{(n)}, \ldots, A_{4}^{(n)}$ in $[0, T]^{2}$ for the values of the process $Z^{(n)}$.

(1) We can solve the DBSVIE on a set

$$
A_{1}^{(n)}:=\left\{\left(t_{i}, t_{j}\right) \in\left\{t_{0}, \ldots, t_{n}\right\}^{2}: t_{i}, t_{j} \geq t_{i_{0}}\right\}
$$

for an appropriate $t_{i_{0}}$, see below.

Consider a (discrete time) process $y^{(n)} \in L_{\mathbb{F}^{(n)}}^{2}\left(0, T ; \mathbb{R}^{k}\right)$ and the equation

$$
\lambda_{t_{i}, t_{p}}^{(n), y^{(n)}}=f\left(W^{(n)} ; t_{i}\right)-\frac{T}{n} \sum_{j=p}^{n-1} h\left(t_{j}, y_{t_{j}}^{(n)}\right)-\sum_{j=p}^{n-1} Z_{t_{i}, t_{j}}^{(n), y^{(n)}} \Delta W_{t_{j+1}}^{(n)} .
$$

This is a family of discrete BSDEs parameterized by $t_{i} \in\left\{t_{i_{0}}, \ldots, t_{n}\right\}$ which can be solved uniquely for each $t_{i}$ on $A_{1}^{(n)}$ due to Lemma 2.2.1 if $n$ is 


\section{PROOFS}

sufficiently large. Especially for $t_{i}=t_{p}$ we obtain a unique solution of the discrete BSVIE of the form

$$
Y_{t_{i}}^{(n), y^{(n)}}=f\left(W^{(n)} ; t_{i}\right)-\frac{T}{n} \sum_{j=i}^{n-1} h\left(t_{j}, y_{t_{j}}^{(n)}\right)-\sum_{j=i}^{n-1} Z_{t_{i}, t_{j}}^{(n), y^{(n)}} \Delta W_{t_{j+1}}^{(n)}
$$

In addition, the map $\theta^{(n)}: \mathcal{H}^{2,(n)}[0, T] \rightarrow \mathcal{H}^{2,(n)}[0, T]$ which maps

$$
\left(y^{(n)}, z^{(n)}\right) \mapsto\left(Y^{(n), y^{(n)}}, Z^{(n), y^{(n)}}\right)
$$

is a contraction, if $T-t_{i_{0}}$ is small enough. To show this remark that taking two different solutions $\left(Y^{(n), y^{(n)}}, Z^{(n), y^{(n)}}\right)$ and $\left(Y^{(n), \tilde{y}^{(n)}}, Z^{(n), \tilde{y}^{(n)}}\right)$ of 2.3 one obtains

$$
\begin{gathered}
\left\|Y_{t_{i}}^{(n), y^{(n)}}-Y_{t_{i}}^{(n), \tilde{y}^{(n)}}\right\|_{2}^{2}=\left\|\mathbb{E}\left[Y_{t_{i}}^{(n), y^{(n)}}-Y_{t_{i}}^{(n), \tilde{y}^{(n)}} \mid \mathcal{F}_{t_{i}}^{(n)}\right]\right\|_{2}^{2} \\
=\left.\left(\frac{T}{n}\right)^{2}\left\|\mathbb{E}\left[\sum_{j=i}^{n-1}\left(h\left(t_{j}, y_{t_{j}}^{(n)}\right)-h\left(t_{j}, \tilde{y}_{t_{j}}^{(n)}\right)\right) \mid \mathcal{F}_{t_{i}}^{(n)}\right]\right\|\right|_{2} ^{2} \\
\leq\left(\frac{T}{n}\right)^{2} \mathbb{E}\left[\left.\left\|\sum_{j=i}^{n-1}\left(h\left(t_{j}, y_{t_{j}}^{(n)}\right)-h\left(t_{j}, \tilde{y}_{t_{j}}^{(n)}\right)\right)\right\|_{2}\right|_{\mid} ^{2} \mathcal{F}_{t_{i}}^{(n)}\right] \\
\leq\left(\frac{L T}{n}\right)^{2} \mathbb{E}\left[\left.2(n-i) \sum_{j=i}^{n-1}\left\|y_{t_{j}}^{(n)}-\tilde{y}_{t_{j}}^{(n)}\right\|_{2}^{2}\right|_{\left.\mathcal{F}_{t_{i}}^{(n)}\right]}\right.
\end{gathered}
$$

and, thus,

$$
\begin{aligned}
& \left\|Y^{(n), y^{(n)}}-Y^{(n), \tilde{y}^{(n)}}\right\|_{L_{\mathbb{F}}^{2}(n)}\left(t_{i_{0}}, t_{n} ; \mathbb{R}^{k}\right)=\left(\mathbb{E}\left[\frac{T}{n} \sum_{i=i_{0}}^{n-1}\left\|Y_{t_{i}}^{(n), y^{(n)}}-Y_{t_{i}}^{(n), \tilde{y}^{(n)}}\right\|_{2}^{2}\right]\right)^{\frac{1}{2}} \\
& \leq \frac{L T}{n}\left(\sum_{i=i_{0}}^{n-1} 2(n-i) \mathbb{E}\left[\frac{T}{n} \sum_{j=i_{0}}^{n-1}\left\|y_{t_{j}}^{(n)}-\tilde{y}_{t_{j}}^{(n)}\right\|_{2}^{2}\right]\right)^{\frac{1}{2}} \\
& \leq \frac{L T}{n}\left(\sum_{i=i_{0}}^{n-1} 2 n\right)^{\frac{1}{2}}\left\|y^{(n)}-\tilde{y}^{(n)}\right\|_{L_{\mathbb{F}}^{2}(n)}\left(t_{i_{0}}, t_{n} ; \mathbb{R}^{k}\right) \\
& =L T \sqrt{\frac{2\left(n-i_{0}\right)}{n}}\left\|y^{(n)}-\tilde{y}^{(n)}\right\|_{L_{\mathbb{F}^{(n)}}^{2}\left(t_{i_{0}}, t_{n} ; \mathbb{R}^{k}\right)} .
\end{aligned}
$$


Next, denoting by $\Delta Z_{i, j}^{(n)}:=Z_{t_{i}, t_{j}}^{(n), y^{(n)}}-Z_{t_{i}, t_{j}}^{(n) \tilde{y}^{(n)}}$ and $\Delta Y_{i}^{(n)}:=Y_{t_{i}}^{(n), y^{(n)}}-$ $Y_{t_{i}}^{(n), \tilde{y}^{(n)}}$ we obtain

$\mathbb{E}\left[\left\|\sum_{j=i_{0}}^{n-1} \Delta Z_{i, j}^{(n)} \Delta W_{t_{j+1}}^{(n)}\right\|_{2}^{2}\right]$

$=\mathbb{E}\left[\left(\sum_{J=1}^{d} \sum_{j=i_{0}}^{n-1} \Delta Z_{i, j}^{1 J,(n)} \Delta W_{t_{j+1}}^{J,(n)}\right)^{2}+\ldots+\left(\sum_{J=1}^{d} \sum_{j=i_{0}}^{n-1} \Delta Z_{i, j}^{k J,(n)} \Delta W_{t_{j+1}}^{J,(n)}\right)^{2}\right]$

$=\frac{T}{n} \sum_{j=i_{0}}^{n-1} \mathbb{E}\left[\sum_{J=1}^{d}\left(\Delta Z_{i, j}^{1 J,(n)}\right)^{2}+\ldots+\sum_{J=1}^{d}\left(\Delta Z_{i, j}^{k J,(n)}\right)^{2}\right]=\frac{T}{n} \sum_{j=i_{0}}^{n-1} \mathbb{E}\left[\left\|\Delta Z_{i, j}^{(n)}\right\|_{2}^{2}\right]$,

since, using the martingale property of the random walk, it holds that for $\tilde{k} \in\{1, \ldots, k\}$

$\mathbb{E}\left[\left(\sum_{J=1}^{d} \sum_{j=i_{0}}^{n-1} \Delta Z_{i, j}^{\tilde{k} J,(n)} \Delta W_{t_{j+1}}^{J,(n)}\right)^{2}\right]=\mathbb{E}\left[\sum_{J=1}^{d}\left(\sum_{j=i_{0}}^{n-1} \Delta Z_{i, j}^{\tilde{k} J,(n)} \Delta W_{t_{j+1}}^{J,(n)}\right)^{2}\right.$
$\left.+2 \sum_{\substack{J, \tilde{J}=1 \\ J<\tilde{J}}}^{d}\left(\sum_{j=i_{0}}^{n-1} \Delta Z_{i, j}^{\tilde{k} J,(n)} \Delta W_{t_{j+1}}^{J,(n)} \cdot \sum_{j=i_{0}}^{n-1} \Delta Z_{i, j}^{\tilde{k} \tilde{J},(n)} \Delta W_{t_{j+1}}^{\tilde{J},(n)}\right)\right]$

$=\sum_{J=1}^{d} \sum_{j=i_{0}}^{n-1} \mathbb{E}\left[\left(\Delta Z_{i, j}^{\tilde{k} J,(n)}\right)^{2}\right] \Delta t_{j+1}$

$$
\begin{aligned}
+2 \sum_{\substack{J, \tilde{J}=1 \\
J<\tilde{J}}}^{d} \sum_{j=i_{0}}^{n-1} \mathbb{E}\left[\Delta Z_{i, j}^{\tilde{k} J,(n)} \Delta Z_{i, j}^{\tilde{k} \tilde{J},(n)} \Delta W_{t_{j+1}}^{J,(n)} \Delta W_{t_{j+1}}^{\tilde{J},(n)}\right] \\
=\frac{T}{n} \sum_{j=i_{0}}^{n-1} \mathbb{E}\left[\sum_{J=1}^{d}\left(\Delta Z_{i, j}^{\tilde{k} J,(n)}\right)^{2}\right] .
\end{aligned}
$$

As $\left(Y^{(n), y^{(n)}}, Z^{(n), y^{(n)}}\right)$ and $\left(Y^{(n), \tilde{y}^{(n)}}, Z^{(n), \tilde{y}^{(n)}}\right)$ are M-solutions, using martingale representation 1.11$)$ and $(2.3)$

$$
\begin{gathered}
\left\|Z^{(n), y^{(n)}}-Z^{(n), \tilde{y}^{(n)}}\right\|_{L^{2,(n)}\left(t_{i_{0}}, t_{n} ; \mathbb{R}^{k \times d}\right)}=\left(\mathbb{E}\left[\left(\frac{T}{n}\right)^{2} \sum_{i=i_{0}}^{n-1} \sum_{j=i_{0}}^{n-1}\left\|\Delta Z_{i, j}^{(n)}\right\|_{2}^{2}\right]\right)^{\frac{1}{2}} \\
=\left(\frac{T}{n} \sum_{i=i_{0}}^{n-1} \mathbb{E}\left[\left\|\sum_{j=i_{0}}^{i-1} \Delta Z_{i, j}^{(n)} \Delta W_{t_{j+1}}^{(n)}+\sum_{j=i}^{n-1} \Delta Z_{i, j}^{(n)} \Delta W_{t_{j+1}}^{(n)}\right\|_{2}^{2}\right]\right)^{\frac{1}{2}}
\end{gathered}
$$




\section{PROOFS}

$$
\begin{aligned}
& =\left(\frac { T } { n } \sum _ { i = i _ { 0 } } ^ { n - 1 } \mathbb { E } \left[\| \Delta Y_{i}^{(n)}-\mathbb{E}\left[\Delta Y_{i}^{(n)} \mid \mathcal{F}_{t_{i_{0}}}^{(n)}\right]\right.\right. \\
& \left.\left.\quad-\Delta Y_{i}^{(n)}-\frac{T}{n} \sum_{j=i}^{n-1}\left(h\left(t_{j}, y_{t_{j}}^{(n)}\right)-h\left(t_{j}, \tilde{y}_{t_{j}}^{(n)}\right)\right) \|_{2}\right]\right)^{2} \\
& \leq\left(2 \mathbb{E}\left[\frac{T}{n} \sum_{i=i_{0}}^{n-1}\left\|\Delta Y_{i}^{(n)}\right\|_{2}^{2}\right]\right)^{\frac{1}{2}} \leq L T \sqrt{\frac{4\left(n-i_{0}\right)}{n}}\left\|y^{(n)}-\tilde{y}^{(n)}\right\|_{L_{\mathbb{F}^{2}(n)}^{2}}\left(t_{i_{0}}, t_{n} ; \mathbb{R}^{k}\right) .
\end{aligned}
$$

At last, we can estimate

$$
\begin{aligned}
\left\|\left(Y^{(n), y^{(n)}}-Y^{(n), \tilde{y}^{(n)}}, Z^{(n), y^{(n)}}-Z^{\left.(n), \tilde{y}^{(n)}\right)}\right)\right\|_{\mathcal{H}^{2,(n)}\left[t_{i_{0}}, t_{n}\right]} \\
\quad \leq L T \sqrt{\frac{6\left(n-i_{0}\right)}{n}}\left\|y^{(n)}-\tilde{y}^{(n)}\right\|_{L_{\mathbb{F}^{(n)}}^{2}\left(t_{i_{0}}, t_{n} ; \mathbb{R}^{k}\right)} .
\end{aligned}
$$

For $n$ large and $n-i_{0}$ small enough we obtain $L T \sqrt{\frac{6\left(n-i_{0}\right)}{n}}<1$, from which it follows that $\theta^{(n)}$ is a contraction on $\mathcal{H}^{2,(n)}\left[t_{i_{0}}, T\right]$ and, therefore, one can solve Equation 1.10 on the set $A_{1}^{(n)}$ with solution $\left(Y^{(n)}, Z^{(n)}\right)$ in $\mathcal{H}^{2,(n)}\left[t_{i_{0}}, T\right]$.

Note, further, that the solution $\left(Y^{(n), y^{(n)}}, Z^{(n), y^{(n)}}\right)$ lies in $\mathcal{H}^{2,(n)}\left[t_{i_{0}}, T\right]$ because for all solutions $\left(\lambda_{t_{i}, t_{p}}^{(n), y^{(n)}}, Z_{t_{i}, t_{p}}^{(n), y^{(n)}}\right)_{p \in\left\{i_{0}, \ldots, n\right\}}$ of the family of discrete BSDEs (2.2) it holds for all $i \in\left\{i_{0}, \ldots, n\right\}$

$$
\mathbb{E}\left[\frac{T}{n} \sum_{p=i_{0}}^{n-1}\left(\left\|\lambda_{t_{i}, t_{p}}^{(n), y^{(n)}}\right\|_{2}^{2}+\left\|Z_{t_{i}, t_{p}}^{(n), y^{(n)}}\right\|_{2}^{2}\right)\right]<\infty .
$$

(2) Discrete martingale representation theorem, see Theorem 1.1.2, determines $Z_{t_{i}, t_{j}}^{(n)}$ on

$$
A_{2}^{(n)}:=\left\{\left(t_{i}, t_{j}\right) \in\left\{t_{0}, \ldots, t_{n}\right\}^{2}: t_{i} \geq t_{i_{0}}, t_{j} \in\left\{t_{0}, \ldots, t_{i_{0}-1}\right\}\right\} .
$$

In addition, from the previous step

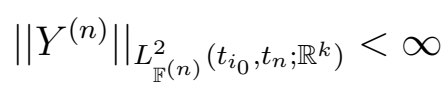

and, thus,

$$
\mathbb{E}\left[\left(\frac{T}{n}\right)^{2} \sum_{i=i_{0}}^{n-1} \sum_{j=0}^{i_{0}-1}\left\|Z_{t_{i}, t_{j}}^{(n)}\right\|_{2}^{2}\right] \leq \mathbb{E}\left[\left(\frac{T}{n}\right)^{2} \sum_{i=i_{0}}^{n-1} \sum_{j=0}^{i-1}\left\|Z_{t_{i}, t_{j}}^{(n)}\right\|_{2}^{2}\right]
$$




$$
\begin{aligned}
= & \mathbb{E}\left[\frac{T}{n} \sum_{i=i_{0}}^{n-1}\left\|\sum_{j=0}^{i-1} Z_{t_{i}, t_{j}}^{(n)} \Delta W_{t_{j+1}}^{(n)}\right\|_{2}^{2}\right]=\mathbb{E}\left[\frac{T}{n} \sum_{i=i_{0}}^{n-1}\left\|Y_{t_{i}}^{(n)}-\mathbb{E}\left[Y_{t_{i}}^{(n)} \mid \mathcal{F}_{t_{0}}^{(n)}\right]\right\|_{2}^{2}\right] \\
& \leq \mathbb{E}\left[\frac{T}{n} \sum_{i=i_{0}}^{n-1}\left\|Y_{t_{i}}^{(n)}\right\|_{2}^{2}\right]=\left\|Y^{(n)}\right\|_{L_{\mathbb{F}(n)}^{2}\left(t_{i_{0}}, t_{n} ; \mathbb{R}^{k}\right)}^{2}<\infty
\end{aligned}
$$

(3) $Z_{t_{i}, t_{j}}^{(n)}$ and $Y_{t_{i}}^{(n)}$ are already determined for $\left(t_{i}, t_{j}\right) \in\left\{t_{i_{0}}, \ldots, t_{n}\right\} \times\left\{t_{0}, \ldots, t_{n}\right\}$. To compute $Z_{t_{i}, t_{j}}^{(n)}$ on

$$
A_{3}^{(n)}:=\left\{\left(t_{i}, t_{j}\right) \in\left\{t_{0}, \ldots, t_{n}\right\}^{2}: t_{i} \in\left\{t_{0}, \ldots, t_{i_{0}-1}\right\}, t_{j} \in\left\{t_{i_{0}}, \ldots, t_{n}\right\}\right\}
$$

consider the equation

$$
\psi_{t_{i}, t_{j}}=f\left(W^{(n)} ; t_{i}\right)-\sum_{p=j}^{n-1} h\left(t_{p}, Y_{t_{p}}^{(n)}\right)-\sum_{p=j}^{n-1} Z_{t_{i}, t_{p}}^{(n)} \Delta W_{t_{p+1}}^{(n)}
$$

This is a family of discrete BSDE parameterized by $t_{i} \in\left\{t_{0}, \ldots, t_{i_{0}-1}\right\}$. For each $t_{i}$ it has a unique solution $\left(\psi_{t_{i}, t_{j}}, Z_{t_{i}, t_{j}}^{(n)}\right)_{j \in\left\{i_{0}, \ldots, n\right\}}$ with

$$
\mathbb{E}\left[\sum_{i=0}^{i_{0}-1} \sum_{j=i_{0}}^{n-1}\left\|Z_{t_{i}, t_{j}}^{(n)}\right\|_{2}^{2}\right]<\infty
$$

as for all $i \in\left\{0, \ldots, i_{0}\right\}$

$$
\mathbb{E}\left[\frac{T}{n} \sum_{j=i_{0}}^{n-1}\left(\left\|\psi_{t_{i}, t_{j}}\right\|_{2}^{2}+\left\|Z_{t_{i}, t_{j}}^{(n)}\right\|_{2}^{2}\right)\right]<\infty
$$

using that $\left\|Y^{(n)}\right\|_{L_{\mathbb{F}}^{2}(n)}\left(t_{i_{0}}, t_{n} ; \mathbb{R}^{k}\right)<\infty$.

(4) Continue by induction considering for $i<i_{0}$

$$
Y_{t_{i}}^{(n)}=\psi_{t_{i}, t_{i_{0}}}-\sum_{p=i}^{i_{0}-1} h\left(t_{p}, Y_{t_{p}}^{(n)}\right)-\sum_{p=i}^{i_{0}-1} Z_{t_{i}, t_{p}}^{(n)} \Delta W_{t_{p+1}}^{(n)} .
$$




\section{PROOFS}

\subsection{Estimations for BSVIEs and DBSVIEs}

The next lemma estimates the difference of solutions $Y$ and $\bar{Y}$ of Equation (1.9), if $Y(\bar{Y})$ has the free term $f(\bar{f})$ and the generator $h(\bar{h})$.

Lemma 2.3.1. Let $f, \bar{f}$ be two free terms and $h, \bar{h}$ be two generators satisfying $(\mathbf{V 1})$ and $(\mathbf{V 2})$. Let $(Y, Z)$ and $(\bar{Y}, \bar{Z})$ be two adapted $M$-solutions of $(1.9)$ with $f$ and $h$ replaced by $\bar{f}$ and $\bar{h}$, respectively. Then it holds

$$
\|Y-\bar{Y}\|_{L_{\mathbb{F}}^{2, \infty}\left(0, T ; \mathbb{R}^{k}\right)} \leq \sqrt{C\left(\mathbb{E}\left[\|f-\bar{f}\|_{\infty}^{2}\right]+\|h-\bar{h}\|_{\infty}^{2}\right)}
$$

for some constant $C<\infty$ depending only on the Lipschitz constant of $h$ and $\bar{h}$, where

$$
\|f-\bar{f}\|_{\infty}(\omega):=\sup _{s \in[0, T]}\|f(\omega ; s)-\bar{f}(\omega ; s)\|_{2}
$$

and

$$
\|h-\bar{h}\|_{\infty}:=\sup _{(s, y) \in[0, T] \times \mathbb{R}^{k}}\|h(s, y)-\bar{h}(s, y)\|_{2} .
$$

Proof. Notice that

$$
\left\|Y_{t}-\bar{Y}_{t}\right\|_{2} \leq \mathbb{E}\left[\|f-\bar{f}\|_{\infty}+\int_{0}^{T}\left\|h\left(s, Y_{s}\right)-\bar{h}\left(s, \bar{Y}_{s}\right)\right\|_{2} d s \mid \mathcal{F}_{t}\right]:=N_{t} .
$$

$N_{t}$ is a martingale, since for all times $t_{1}$ and $t_{2}$ with $t_{1}>t_{2}$ it fulfills

$$
\mathbb{E}\left[N_{t_{1}} \mid \mathcal{F}_{t_{2}}\right]=\mathbb{E}\left[\|f-\bar{f}\|_{\infty}+\int_{0}^{T}\left\|h\left(s, Y_{s}\right)-\bar{h}\left(s, \bar{Y}_{s}\right)\right\|_{2} d s \mid \mathcal{F}_{t_{2}}\right] .
$$

Also, from

$$
\begin{aligned}
\left\|h\left(s, Y_{s}\right)-\bar{h}\left(s, \bar{Y}_{s}\right)\right\|_{2} \leq\left\|h\left(s, Y_{s}\right)-h\left(s, \bar{Y}_{s}\right)\right\|_{2}+\left\|h\left(s, \bar{Y}_{s}\right)-\bar{h}\left(s, \bar{Y}_{s}\right)\right\|_{2} & \\
& \leq L\left\|Y_{s}-\bar{Y}_{s}\right\|_{2}+\|h-\bar{h}\|_{\infty}
\end{aligned}
$$

one obtains that

$$
\left\|h\left(s, Y_{s}\right)-\bar{h}\left(s, \bar{Y}_{s}\right)\right\|_{2}^{2} \leq 2\left(L^{2}\left\|Y_{s}-\bar{Y}_{s}\right\|_{2}^{2}+\|h-\bar{h}\|_{\infty}^{2}\right)
$$

and using the properties for the conditional expectation

$$
\mathbb{E}\left[\left\|Y_{t}-\bar{Y}_{t}\right\|_{2}^{2}\right] \leq \mathbb{E}\left[N_{t}^{2}\right] \leq 2 \mathbb{E}\left[\|f-\bar{f}\|_{\infty}^{2}+T \int_{0}^{T}\left\|h\left(s, Y_{s}\right)-\bar{h}\left(s, \bar{Y}_{s}\right)\right\|_{2}^{2} d s\right]
$$




$$
\begin{array}{r}
\leq 2 \mathbb{E}\left[\|f-\bar{f}\|_{\infty}^{2}\right]+4 T \mathbb{E}\left[\int_{0}^{T}\left(L^{2}\left\|Y_{s}-\bar{Y}_{s}\right\|_{2}^{2}+\|h-\bar{h}\|_{\infty}^{2}\right) d s\right] \\
\leq C_{1}+4 T L^{2} \mathbb{E}\left[\int_{0}^{T}\left\|Y_{s}-\bar{Y}_{s}\right\|_{2}^{2} d s\right]
\end{array}
$$

with

$$
\begin{aligned}
C_{1}:=2 \mathbb{E}\left[\|f-\bar{f}\|_{\infty}^{2}\right]+4 T \int_{0}^{T}\|h-\bar{h}\|_{\infty}^{2} d s & \\
& =2 \mathbb{E}\left[\|f-\bar{f}\|_{\infty}^{2}\right]+4 T^{2}\|h-\bar{h}\|_{\infty}^{2} .
\end{aligned}
$$

Moreover, by Gronwall's inequality in [21]

$$
\mathbb{E}\left[\left\|Y_{t}-\bar{Y}_{t}\right\|_{2}^{2}\right] \leq C_{1} e^{\int^{T} 4 T L^{2} d s}=C_{1} e^{(T-t) 4 T L^{2}} \leq C_{1} e^{4 T^{2} L^{2}},
$$

and using Doob's inequality for martingales from [16] given in Theorem 7.31 one obtains

$$
\begin{gathered}
\|Y-\bar{Y}\|_{L_{\mathbb{F}}^{2, \infty}\left(0, T ; \mathbb{R}^{k}\right)}^{2}=\mathbb{E}\left[\sup _{t \in[0, T]}\left\|Y_{t}-\bar{Y}_{t}\right\|_{2}^{2}\right] \leq \mathbb{E}\left[\sup _{t \in[0, T]} N_{t}^{2}\right] \leq 4 \mathbb{E}\left[N_{T}^{2}\right] \\
\leq 8 \mathbb{E}\left[\|f-\bar{f}\|_{\infty}^{2}\right]+16 T^{2}\|h-\bar{h}\|_{\infty}^{2}+16 T L^{2} \int_{0}^{T} \mathbb{E}\left[\left\|Y_{s}-\bar{Y}_{s}\right\|_{2}^{2}\right] d s \\
\leq 8 \mathbb{E}\left[\|f-\bar{f}\|_{\infty}^{2}\right]+16 T^{2}\|h-\bar{h}\|_{\infty}^{2}+16 T^{2} L^{2} e^{4 T^{2} L^{2} C_{1}} \\
\leq C\left(\mathbb{E}\left[\|f-\bar{f}\|_{\infty}^{2}\right]+\|h-\bar{h}\|_{\infty}^{2}\right),
\end{gathered}
$$

for some finite constant $C$ depending only on $L$ and $T$.

The next lemma is the analogous version of Lemma 2.3.1 in the discrete case.

Lemma 2.3.2. Let $f, \bar{f}$ be two free terms and $h, \bar{h}$ be two generators satisfying (D1) and (D2). Let $\left(Y^{(n)}, Z^{(n)}\right)_{n \in \mathbb{N}}$ and $\left(\bar{Y}^{(n)}, \bar{Z}^{(n)}\right)_{n \in \mathbb{N}}$ be two discrete $M$ solutions of 1.10 with $f$ and $h$ replaced by $\bar{f}$ and $\bar{h}$, respectively. Then it holds

$$
\limsup _{n \rightarrow \infty}\left\|Y^{(n)}-\bar{Y}^{(n)}\right\|_{L_{\mathbb{F}}^{2, \infty}\left(0, T ; \mathbb{R}^{k}\right)} \leq \sqrt{C\left(\mathbb{E}\left[\|f-\bar{f}\|_{\infty}^{2}\right]+\|h-\bar{h}\|_{\infty}^{2}\right)}
$$




\section{PROOFS}

for some constant $C<\infty$ depending only on the Lipschitz constant of $h$ and $\bar{h}$, where

$$
\|f-\bar{f}\|_{\infty}(\omega):=\sup _{s \in[0, T]}\|f(\omega ; s)-\bar{f}(\omega ; s)\|_{2}
$$

and

$$
\|h-\bar{h}\|_{\infty}:=\sup _{(s, y) \in[0, T] \times \mathbb{R}^{k}}\|h(s, y)-\bar{h}(s, y)\|_{2} .
$$

Proof. The proof is the discrete analogon to the proof of Lemma 2.3.1, i.e. we estimate $\left\|Y_{t_{i}}^{(n)}-\bar{Y}_{t_{i}}^{(n)}\right\|_{2}$ with the martingale

$$
N_{t_{i}}:=\mathbb{E}\left[\|f-\bar{f}\|_{\infty}+\frac{T}{n} \sum_{j=0}^{n-1}\left\|h\left(t_{j}, Y_{t_{j}}^{(n)}\right)-\bar{h}\left(t_{j}, \bar{Y}_{t_{j}}^{(n)}\right)\right\|_{2} \mid \mathcal{F}_{t_{i}}^{(n)}\right]
$$

and apply the discrete Gronwall inequality and Doob's inequality together with the fact that $W^{(n)}$ converges weakly to $W$ by Donsker's Theorem to show the required estimation.

\subsection{Tightness}

To prove the weak convergence of the sequence $\left(Y^{(n)}\right)_{n \in \mathbb{N}}$ from 1.10 in the Skorokhod topology we have to show that this sequence is tight.

Lemma 2.4.1. Assume that the assumptions (V1)-(V4) are fulfilled. Let $\left(Y^{(n)}, Z^{(n)}\right)_{n \in \mathbb{N}}$ be the sequence of discrete M-solutions from 1.10). Then the sequence $\left(Y^{(n)}\right)_{n \in \mathbb{N}}$ is tight in the Skorokhod topology.

For the proof we need the next lemma.

Lemma 2.4.2. The jumps of $Y^{(n)}$ in 1.10 converge uniformly to zero, more precisely, it holds

$$
\left\|Y_{t_{i+1}}^{(n)}-Y_{t_{i}}^{(n)}\right\|_{2} \leq \frac{K e^{2 L}}{\sqrt{n}}+\frac{K T+D}{n} \quad \mathbb{P} \text {-a.s. }
$$

Proof. From Chapter 1, Subsection 1.2.1, we can interpret (1.9) as a parameterized BSDE in the following sense:

$$
Y_{t, r}=f(W ; t)-\int_{r}^{T} h\left(s, Y_{s, s}\right) d s-\int_{r}^{T} Z_{t, s} d W_{s}, r \in[t, T],
$$

with $Y_{t, \cdot}, Z_{t, \cdot}$ being $\mathbb{F}$-adapted for almost all $t \in[0, T]$ and $Y_{s}:=Y_{s, s}$. 
From above we can write the following discretization of the solution $Y_{t, r}$ :

$$
Y_{t_{i}, t_{k}}^{(n)}=f\left(W^{(n)} ; t_{i}\right)-\frac{T}{n} \sum_{j=k}^{n-1} h\left(t_{j}, Y_{t_{j}, t_{j}}^{(n)}\right)-\sum_{j=k}^{n-1} Z_{t_{i}, t_{j}}^{(n)} \Delta W_{t_{j+1}}^{(n)}
$$

and, in particular, $Y_{t_{i}, t_{i}}^{(n)}$ coincide with 1.10 .

Thus, we obtain

$$
\begin{aligned}
\left\|Y_{t_{i+1}}^{(n)}-Y_{t_{i}}^{(n)}\right\|_{2} & =\left\|Y_{t_{i+1}, t_{i+1}}^{(n)}-Y_{t_{i}, t_{i+1}}^{(n)}+Y_{t_{i}, t_{i+1}}^{(n)}-Y_{t_{i}, t_{i}}^{(n)}\right\|_{2} \\
& \leq\left\|Y_{t_{i+1}, t_{i+1}}^{(n)}-Y_{t_{i}, t_{i+1}}^{(n)}\right\|_{2}+\left\|Y_{t_{i}, t_{i+1}}^{(n)}-Y_{t_{i}, t_{i}}^{(n)}\right\|_{2}=: A+B,
\end{aligned}
$$

where due to adaptiveness of $Y^{(n)}, Z_{t_{i+1},}^{(n)}, Z_{t_{i}}^{(n)}$.

$$
\begin{gathered}
A=\left\|\mathbb{E}\left[Y_{t_{i+1}, t_{i+1}}^{(n)}-Y_{t_{i}, t_{i+1}}^{(n)} \mid \mathcal{F}_{t_{i+1}}^{(n)}\right]\right\|_{2} \\
=\| \mathbb{E}\left[f\left(W^{(n)} ; t_{i+1}\right)-\frac{T}{n} \sum_{j=i+1}^{n-1} h\left(t_{j}, Y_{t_{j}}^{(n)}\right)-\sum_{j=i+1}^{n-1} Z_{t_{i+1}, t_{j}}^{(n)} \Delta W_{t_{j+1}}^{(n)}\right. \\
\left.\quad-f\left(W^{(n)} ; t_{i}\right)+\frac{T}{n} \sum_{j=i+1}^{n-1} h\left(t_{j}, Y_{t_{j}}^{(n)}\right)+\sum_{j=i+1}^{n-1} Z_{t_{i}, t_{j}}^{(n)} \Delta W_{t_{j+1}}^{(n)} \mid \mathcal{F}_{t_{i+1}}^{(n)}\right] \|_{2} \\
\leq\left\|\mathbb{E}\left[f\left(W^{(n)} ; t_{i+1}\right)-f\left(W^{(n)} ; t_{i}\right) \mid \mathcal{F}_{t_{i+1}}^{(n)}\right]\right\|_{2} \\
\leq K\left|t_{i+1}-t_{i}\right|=\frac{K T}{n}
\end{gathered}
$$

and, using Lemma 3.2 in [18] for the parameter $r=t_{i}$,

$$
B \leq \frac{K e^{2 L}}{\sqrt{n}}+\frac{D}{n}
$$

Hence, for all $i=0, \ldots, n$,

$$
\left\|Y_{t_{i+1}}^{(n)}-Y_{t_{i}}^{(n)}\right\|_{2} \leq \frac{K e^{2 L}}{\sqrt{n}}+\frac{K T+D}{n},
$$

which proves the lemma.

Proof of Lemma 2.4.1. Consider the following decomposition for the discretized solution $Y_{t_{i}}^{(n)}, i=0, \ldots, n$ :

$$
Y_{t_{i}}^{(n)}=U_{t_{i}}^{(n)}+A_{t_{i}}^{(n)}
$$




\section{PROOFS}

where

$$
U_{t_{i}}^{(n)}:=\sum_{j=0}^{i-1}\left(Y_{t_{j+1}}^{(n)}-\mathbb{E}\left[Y_{t_{j+1}}^{(n)} \mid \mathcal{F}_{t_{j}}^{(n)}\right]\right)
$$

and

$$
A_{t_{i}}^{(n)}:=Y_{t_{i}}^{(n)}-U_{t_{i}}^{(n)}
$$

It holds that $A^{(n)}$ is a predictable process with finite variation (and this variation is bounded independently of $n$ ), because using tower property of conditional expectation to the sum with $j \leq i-2$ we obtain that

$$
\begin{gathered}
A_{t_{i}}^{(n)}=Y_{t_{i}}^{(n)}-\sum_{j=0}^{i-1}\left(Y_{t_{j+1}}^{(n)}-\mathbb{E}\left[Y_{t_{j+1}}^{(n)} \mid \mathcal{F}_{t_{j}}^{(n)}\right]\right) \\
=Y_{t_{i}}^{(n)}-Y_{t_{i}}^{(n)}+\mathbb{E}\left[Y_{t_{i}}^{(n)} \mid \mathcal{F}_{t_{i-1}}^{(n)}\right]-\sum_{j=0}^{i-2}\left(Y_{t_{j+1}}^{(n)}-\mathbb{E}\left[Y_{t_{j+1}}^{(n)} \mid \mathcal{F}_{t_{j}}^{(n)}\right]\right) \\
=\mathbb{E}\left[Y_{t_{i}}^{(n)} \mid \mathcal{F}_{t_{i-1}}^{(n)}\right]-\sum_{j=0}^{i-2}\left(Y_{t_{j+1}}^{(n)}-\mathbb{E}\left[Y_{t_{j+1}}^{(n)} \mid \mathcal{F}_{t_{j}}^{(n)}\right]\right)
\end{gathered}
$$

is $\mathcal{F}_{t_{i-1}}^{(n)}$-measurable and

$$
\begin{aligned}
& \sum_{i=0}^{n-1}\left\|A_{t_{i+1}}^{(n)}-A_{t_{i}}^{(n)}\right\|_{2} \\
& =\sum_{i=0}^{n-1} \| \mathbb{E}\left[Y_{t_{i+1}}^{(n)} \mid \mathcal{F}_{t_{i}}^{(n)}\right]-\sum_{j=0}^{i-1}\left(Y_{t_{j+1}}^{(n)}-\mathbb{E}\left[Y_{t_{j+1}}^{(n)} \mid \mathcal{F}_{t_{j}}^{(n)}\right]\right) \\
& -\mathbb{E}\left[Y_{t_{i}}^{(n)} \mid \mathcal{F}_{t_{i-1}}^{(n)}\right]+\sum_{j=0}^{i-2}\left(Y_{t_{j+1}}^{(n)}-\mathbb{E}\left[Y_{t_{j+1}}^{(n)} \mid \mathcal{F}_{t_{j}}^{(n)}\right]\right) \|_{2} \\
& \left.=\sum_{i=0}^{n-1} \| \mathbb{E}\left[f\left(W^{(n)} ; t_{i+1}\right) \mid \mathcal{F}_{t_{i}}^{(n)}\right]-\mathbb{E}\left[f\left(W^{(n)} ; t_{i}\right) \mid \mathcal{F}_{t_{i}}^{(n)}\right]+\frac{T}{n} h\left(Y_{t_{i+1}}^{(n)} \mid \mathcal{F}_{t_{i}}^{(n)}\right]-Y_{t_{i}}^{(n)}\right)\left\|_{t_{i}}^{(n)}\right\|_{2} \\
& \leq \sum_{i=0}^{n-1}\left(\frac{K T}{n}+\frac{D T}{n}\right)=T(K+D),
\end{aligned}
$$

where $K$ and $D$ do not depend on $n$. 
Moreover, $U^{(n)}$ is a square integrable martingale, since $U^{(n)}$ is a martingale, i.e. for any $n \in \mathbb{N}$ we have that

$$
\begin{aligned}
\mathbb{E}\left[U_{t_{i}}^{(n)} \mid \mathcal{F}_{t_{i-1}}^{(n)}\right] & =\sum_{j=0}^{i-1} \mathbb{E}\left[Y_{t_{j+1}}^{(n)} \mid \mathcal{F}_{t_{i-1}}^{(n)}\right]-\sum_{j=0}^{i-1} \mathbb{E}\left[\mathbb{E}\left[Y_{t_{j+1}}^{(n)} \mid \mathcal{F}_{t_{j}}^{(n)}\right] \mid \mathcal{F}_{t_{i-1}}^{(n)}\right] \\
= & \mathbb{E}\left[Y_{t_{i}}^{(n)} \mid \mathcal{F}_{t_{i-1}}^{(n)}\right]+\sum_{j=0}^{i-2} Y_{t_{j+1}}^{(n)}-\mathbb{E}\left[Y_{t_{i}}^{(n)} \mid \mathcal{F}_{t_{i-1}}^{(n)}\right]-\sum_{j=0}^{i-2} \mathbb{E}\left[Y_{t_{j+1}}^{(n)} \mid \mathcal{F}_{t_{j}}^{(n)}\right]=U_{t_{i-1}}^{(n)}
\end{aligned}
$$

and

$$
\begin{aligned}
& \sup _{i} \mathbb{E}\left[\left\|U_{t_{i}}^{(n)}\right\|_{2}^{2}\right] \\
&=\sup _{i}\left(\sum_{j=0}^{i-1} \mathbb{E}\left[|| Y_{t_{j+1}}^{(n)}-\left.\mathbb{E}\left[Y_{t_{j+1}}^{(n)} \mid \mathcal{F}_{t_{j}}^{(n)}\right]\right|_{2} ^{2}\right]\right. \\
&\left.\quad+\sum_{\substack{j, \tilde{j}=0 \\
j \neq \tilde{j}}}^{i-1} \mathbb{E}\left[\left\langle Y_{t_{j+1}}^{(n)}-\mathbb{E}\left[Y_{t_{j+1}}^{(n)} \mid \mathcal{F}_{t_{j}}^{(n)}\right], Y_{t_{\tilde{j}+1}}^{(n)}-\mathbb{E}\left[Y_{t_{\tilde{j}+1}}^{(n)} \mid \mathcal{F}_{t_{\tilde{j}}}^{(n)}\right]\right\rangle\right]\right)<\infty
\end{aligned}
$$

due to boundness of $Y_{t_{i}}^{(n)}$.

Thus, $Y_{t_{i}}^{(n)}$ is a square integrable semimartingale and, as pointed out in [18], we can use Theorem 2.3 (with condition C2) in [13] for the function

$$
G^{(n)}:=\left[U^{(n)}, U^{(n)}\right]+V\left(A^{(n)}\right)
$$

with $\left[U^{(n)}, U^{(n)}\right]$ and $V\left(A^{(n)}\right)$ being the quadratic, resp., total variation of $U^{(n)}$ and $A^{(n)}$.

Namely, one can see that $G^{(n)}$ is bounded by a constant because $V\left(A^{(n)}\right)$ is bounded by $T(K+D)$ not depending on $n$, and using Lemma 2.4 .2 it holds that

$$
\begin{gathered}
{\left[U_{t_{i}}^{(n)}, U_{t_{i}}^{(n)}\right]=\sum_{J=0}^{i-1}\left\|U_{t_{J+1}}^{(n)}-U_{t_{J}}^{(n)}\right\|_{2}^{2}=\sum_{J=0}^{i-1}\left\|Y_{t_{J+1}}^{(n)}-\mathbb{E}\left[Y_{t_{J+1}}^{(n)} \mid \mathcal{F}_{t_{J}}^{(n)}\right]\right\|_{2}^{2}} \\
=\sum_{J=0}^{i-1}\left\|Y_{t_{J+1}}^{(n)}-Y_{t_{J}}^{(n)}-\frac{T}{n} h\left(t_{J}, Y_{t_{J}}^{(n)}\right)+\mathbb{E}\left[f\left(W^{(n)} ; t_{J}\right)-f\left(W^{(n)} ; t_{J+1}\right) \mid \mathcal{F}_{t_{J}}^{(n)}\right]\right\|_{2}^{2} \\
\leq \sum_{J=0}^{i-1}\left(\frac{K e^{2 L}}{\sqrt{n}}+\frac{K T+D}{n}+\frac{T(K+D)}{n}\right)^{2} \leq C
\end{gathered}
$$

for some constant $C$.

Hence, from [13] the sequence $\left(Y^{(n)}\right)$ is relatively compact in the Skorohod topology and, by Prokhorov's theorem, it is tight. 


\section{PROOFS}

\subsection{Weak Convergence in Special Case}

In this section we present the central result to prove the Main Convergence Theorem.

Generalizing the ideas of [18] from BSDEs to BSVIEs, in Subsection 2.5.1 it will be given a method of constructing the M-solutions of $(1.9)$ via solutions of quasilinear PDEs of parabolic type (for general information about the theory of PDEs of parabolic type see [11]), if the free term depends only on finitely many points of the trajectory of the Brownian motion and both, the free term and generator, are sufficiently smooth. The problem of smoothness for solutions of such PDEs of parabolic type we solve in Subsection 2.5.2.

Denote by $\tau:=\left(\tau_{0}, \ldots, \tau_{m}\right)$ a partition of $[0, T]$ of length $m$, i.e. $0=\tau_{0}<$ $\tau_{1}<\ldots<\tau_{m}=T$, and consider the BSVIE

$$
Y_{t}^{[m]}=f^{[m]}\left(W_{\tau_{1}}, \ldots, W_{\tau_{m}} ; t\right)-\int_{t}^{T} h^{[m]}\left(s, Y_{s}^{[m]}\right) d s-\int_{t}^{T} Z_{t, s}^{[m]} d W_{s}, t \in[0, T],
$$

where $f^{[m]}: \mathbb{R}^{l} \times[0, T] \rightarrow \mathbb{R}^{k}$ with $l:=d m$ and $h^{[m]}:[0, T] \times \mathbb{R}^{k} \rightarrow \mathbb{R}^{k}$ are given maps.

Similarly, for a given partition $\tau^{(n)}:=\left(\tau_{0}^{(n)}, \ldots, \tau_{m}^{(n)}\right)$ of $[0, T]$ of length $m$ with $\tau_{\lambda}^{(n)}:=\frac{\left\lfloor\tau_{\lambda} n\right\rfloor}{n}, \lambda=0, \ldots, m$, we consider the DBSVIE

$Y_{t_{i}}^{(n),[m]}=f^{[m]}\left(W_{\tau_{1}^{(n)}}^{(n)}, \ldots, W_{\tau_{m}^{(n)}}^{(n)} ; t_{i}\right)-\frac{T}{n} \sum_{j=i}^{n-1} h^{[m]}\left(t_{j}, Y_{t_{j}}^{(n),[m]}\right)-\sum_{j=i}^{n-1} Z_{t_{i}, t_{j}}^{(n),[m]} \Delta W_{t_{j+1}}^{(n)}$.

For simplicity, in the argument of the free terms we sometimes write $\boldsymbol{W}_{\tau_{m}}:=$ $\left(W_{\tau_{1}}, \ldots, W_{\tau_{m}}\right)$ and $\boldsymbol{W}_{\tau_{m}^{(n)}}^{(n)}:=\left(W_{\tau_{1}^{(n)}}^{(n)}, \ldots, W_{\tau_{m}^{(n)}}^{(n)}\right)$.

To obtain the weak convergence of the solution $Y^{(n),[m]}$ from $(2.5)$ to the solution $Y^{[m]}$ from $(2.4)$ we introduce the following assumptions for the free term $f^{[m]}$ and generator $h^{[m]}$ :

(M1) $f^{[m]}$ is a $\mathcal{B}\left(\mathbb{R}^{l}\right) \otimes \mathcal{B}([0, T])$-measurable map such that $f^{[m]}\left(\boldsymbol{W}_{\tau_{m}}(\cdot) ; t\right)$ is $\mathcal{F}_{T^{-}}$ measurable for all $t \in[0, T]$ and it holds $\mathbb{E}\left[\int_{0}^{T}\left\|f^{[m]}\left(\boldsymbol{W}_{\tau_{m}}(\cdot) ; t\right)\right\|_{2}^{2} d t\right]<\infty$.

(M2) $f^{[m]} \in C_{b}^{\infty}\left(\mathbb{R}^{l} \times[0, T] ; \mathbb{R}^{k}\right)$ and $h^{[m]} \in C_{b}^{\infty}\left([0, T] \times \mathbb{R}^{k} ; \mathbb{R}^{k}\right)$.

Then one obtains the following result: 
Lemma 2.5.1. Assume that in (2.4) and (2.5) the assumptions (M1) and (M2) are fulfilled. Let $m \in \mathbb{N},\left(Y^{(n),[m]}, Z^{(n),[m]}\right)_{n \in \mathbb{N}}$ be the sequence of discrete $M$ solutions from (2.5) and $\left(Y^{[m]}, Z^{[m]}\right)$ be the adapted M-solution of (2.4). Then the sequence $\left(Y^{(n),[m]}\right)_{n \in \mathbb{N}}$ converges weakly in the Skorokhod topology to $Y^{[m]}$, if it is piecewise constant interpolated between the points $Y_{t_{i}}^{(n),[m]}$ and $Y_{t_{i+1}}^{(n),[m]}$ for all $i=0, \ldots, n-1$.

The proof of Lemma 2.5.1 is proceeded in Subsection 2.5.3 with preliminaries results in Subsection 2.5.1 and Section 2.4.

Remark 2.5.2. Note that Equation 2.4 and Equation (2.5) are special cases of Equation (1.9) and (1.10), respectively, if the free term $f$ depends only on finitely many points of the trajectory of the Brownian motion $W$. Hence the existence and uniqueness of the adapted M-solutions follow from Theorem 1.2 .9 and Theorem 1.3.4 as well as tightness for the sequence $\left(Y^{(n),[m]}\right)_{n \in \mathbb{N}}$ from Lemma 2.4.1.

\subsubsection{Construction for the Solutions of BSVIEs with PDEs}

For the sake of simplicity take $T=d=k=1$. Further, suppose that the free term $f^{[m]}$ in (2.4) depends only on two points of the trajectory of a Brownian motion, i.e. $\tau_{2}=1$ (and so $m=2$ ), and the assumptions on $f^{[2]}$ and $h^{[2]}$ from (2.4) for the uniqueness and existence of the adapted M-solution $\left(Y^{[2]}, Z^{[2]}\right)$ hold.

Our purpose is to show that the M-solution of (2.4) can be represented via solutions of six systems of PDEs of parabolic type, if $f^{[2]}$ and $h^{[2]}$ are smooth. Each system of PDEs is defined on one of the following subsets of $[0,1]^{2}$ (see Figure 2.2):

$$
\begin{aligned}
& S^{2,2}:=\left\{(t, s) \in[0,1]^{2} \mid t, s \geq \tau_{1}, t \leq s\right\}, \\
& S^{1,2}:=\left\{(t, s) \in[0,1]^{2} \mid t<\tau_{1}, s \geq \tau_{1}\right\}, \\
& S^{1,1}:=\left\{(t, s) \in[0,1]^{2} \mid t<\tau_{1}, s \leq \tau_{1}, t \leq s\right\}, \\
& S_{2,2}:=\left\{(t, s) \in[0,1]^{2} \mid t, s \geq \tau_{1}, t \geq s\right\}, \\
& S_{2,1}:=\left\{(t, s) \in[0,1]^{2} \mid t \geq \tau_{1}, s \leq \tau_{1}\right\}, \\
& S_{1,1}:=\left\{(t, s) \in[0,1]^{2} \mid t<\tau_{1}, s \leq \tau_{1}, t \geq s\right\} .
\end{aligned}
$$




\section{PROOFS}

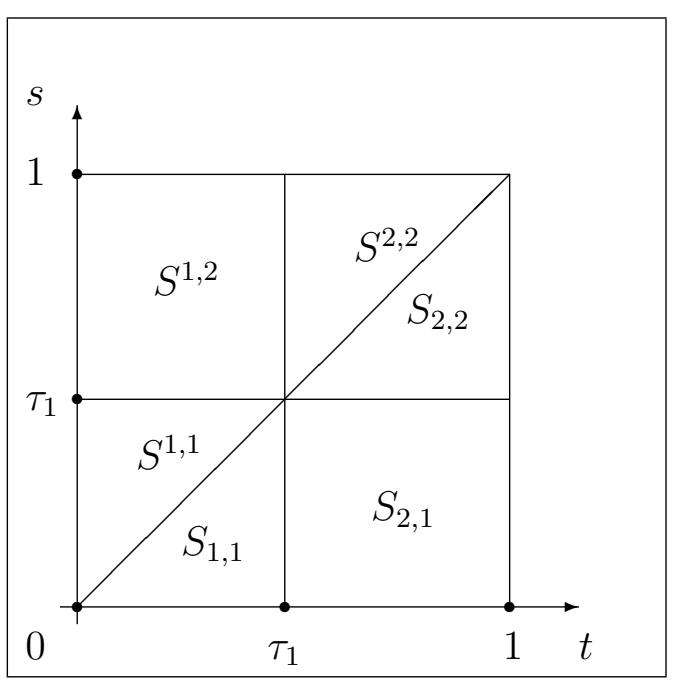

Figure 2.2: The sets $S^{2,2}, \ldots, S_{1,1}$ in $[0,1]^{2}$ for the values of the process $Z^{[2]}$.

To construct PDEs for the upper triangle of $[0,1]^{2}$ we consider $(t, s)$ in all subsets of this domain.

Suppose that $(t, s) \in S^{2,2}$. Then, according to Remark 2.5.2, Equation 2.4 admits an adapted M-solution $\left(Y_{t}^{[2]}, Z_{t, s}^{[2]}\right)$. We consider the ansatz

$$
Y_{t}^{[2]}=u^{2,2}\left(t, W_{\tau_{1}}, W_{t} ; t\right), Z_{t, s}^{[2]}=\frac{\partial u^{2,2}}{\partial y}\left(s, W_{\tau_{1}}, W_{s} ; t\right)
$$

for some deterministic function $u^{2,2}$.

If the partial derivatives

$$
\frac{\partial u^{2,2}}{\partial s}(s, x, y ; t), \frac{\partial^{2} u^{2,2}}{\partial y^{2}}(s, x, y ; t)
$$

exist and are continuous we can apply Itô's formula to $u^{2,2}$ and the Brownian motion, and for $r \in[t, 1]$ get the following equation:

$u^{2,2}\left(1, W_{\tau_{1}}, W_{1} ; t\right)=u^{2,2}\left(r, W_{\tau_{1}}, W_{r} ; t\right)$

$+\int_{r}^{1}\left[\frac{\partial u^{2,2}}{\partial s}\left(s, W_{\tau_{1}}, W_{s} ; t\right)+\frac{1}{2} \frac{\partial^{2} u^{2,2}}{\partial y^{2}}\left(s, W_{\tau_{1}}, W_{s} ; t\right)\right] d s+\int_{r}^{1} \frac{\partial u^{2,2}}{\partial y}\left(s, W_{\tau_{1}}, W_{s} ; t\right) d W_{s}$.

Comparing (2.4) and the previous equation, we obtain that $u^{2,2}$ is required to solve

$$
\left\{\begin{array}{l}
\frac{\partial u^{2,2}}{\partial s}(s, x, y ; t)+\frac{1}{2} \frac{\partial^{2} u^{2,2}}{\partial y^{2}}(s, x, y ; t)=h^{[2]}\left(s, u^{2,2}(s, x, y ; s)\right) \\
u^{2,2}(1, x, y ; t)=f^{[2]}(x, y ; t)
\end{array}\right.
$$


Theorem 2.5.3 below states that for appropriate conditions on the free term $f^{[2]}$ and the generator $h^{[2]}$ a solution $u^{2,2}$ exists and fulfills enough regularity conditions to guarantee that our application of Itô's formula was correct. Therefore, $Y_{t}^{[2]}$ and $Z_{t, s}^{[2]}$ can really be written in terms of $u^{2,2}$ and the Brownian motion.

In a similar way we want to find corresponding systems of PDEs for the other subsets on $[0,1]^{2}$. So in the following we again suppose that $f^{[2]}$ and $h^{[2]}$ fulfill the requirements of Theorem 2.5.3. Then the obtained solutions of the PDE's systems are differentiable enough in order to apply Itô's formula.

For $t \in\left[0, \tau_{1}\right)$ we write 2.4 as follows:

$$
Y_{t}^{[2]}=\psi_{t}^{\tau_{1}}-\int_{t}^{\tau_{1}} h^{[2]}\left(s, Y_{s}^{[2]}\right) d s-\int_{t}^{\tau_{1}} Z_{t, s}^{[2]} d W_{s},
$$

where

$$
\psi_{t}^{\tau_{1}}=f^{[2]}\left(W_{\tau_{1}}, W_{1} ; t\right)-\int_{\tau_{1}}^{1} h^{[2]}\left(s, Y_{s}^{[2]}\right) d s-\int_{\tau_{1}}^{1} Z_{t, s}^{[2]} d W_{s},
$$

which is $\mathcal{F}_{\tau_{1}}$-measurable for almost all $t \in\left[0, \tau_{1}\right)$.

If $(t, s) \in S^{1,2}$ we set

$$
\psi_{t}^{\tau_{1}}=u^{1,2}\left(\tau_{1}, W_{\tau_{1}}, W_{\tau_{1}} ; t\right), Z_{t, s}^{[2]}=\frac{\partial u^{1,2}}{\partial y}\left(s, W_{\tau_{1}}, W_{s} ; t\right),
$$

and, applying Itô's formula, we obtain with equation 2.8 the system

$$
\left\{\begin{array}{l}
\frac{\partial u^{1,2}}{\partial s}(s, x, y ; t)+\frac{1}{2} \frac{\partial^{2} u^{1,2}}{\partial y^{2}}(s, x, y ; t)=h^{[2]}\left(s, u^{2,2}(s, x, y ; s)\right) \\
u^{1,2}(1, x, y ; t)=f^{[2]}(x, y ; t)
\end{array}\right.
$$

For $(t, s) \in S^{1,1}$ set

$$
Y_{t}^{[2]}=u^{1,1}\left(t, W_{t} ; t\right), Z_{t, s}^{[2]}=\frac{\partial u^{1,1}}{\partial z}\left(s, W_{s} ; t\right),
$$

and from 2.7) we get the last system

$$
\left\{\begin{array}{l}
\frac{\partial u^{1,1}}{\partial s}(s, z ; t)+\frac{1}{2} \frac{\partial^{2} u^{1,1}}{\partial z^{2}}(s, z ; t)=h^{[2]}\left(s, u^{1,1}(s, z ; s)\right) \\
u^{1,1}\left(\tau_{1}, z ; t\right)=u^{1,2}\left(\tau_{1}, z, z ; t\right)
\end{array}\right.
$$

Consequently, the solution of (2.4) is given by

$$
Y_{t}^{[2]}= \begin{cases}u^{2,2}\left(t, W_{\tau_{1}}, W_{t} ; t\right) & , \quad t \in\left[\tau_{1}, 1\right] \\ u^{1,1}\left(t, W_{t} ; t\right) & , \quad t \in\left[0, \tau_{1}\right)\end{cases}
$$




$$
Z_{t, s}^{[2]}= \begin{cases}\frac{\partial u^{2,2}}{\partial y}\left(s, W_{\tau_{1}}, W_{s} ; t\right) & ,(t, s) \in S^{2,2} \\ \frac{\partial u^{1,2}}{\partial y}\left(s, W_{\tau_{1}}, W_{s} ; t\right) & ,(t, s) \in S^{1,2} \\ \frac{\partial u^{1,1}}{\partial z}\left(s, W_{s} ; t\right) & , \quad(t, s) \in S^{1,1}\end{cases}
$$

To obtain systems of PDEs for the lower triangle we have to use the Mcondition (1.7), because this condition determines the process $Z_{t, s}^{[2]}$.

Suppose that $(t, s) \in S_{2,2}$, and that from 1.7 with $\mathcal{S}=\tau_{1}$ for a.e. $t \in\left[\tau_{1}, 1\right]$ we have

$$
Y_{t}^{[2]}=\mathbb{E}\left[Y_{t}^{[2]} \mid \mathcal{F}_{\tau_{1}}\right]+\int_{\tau_{1}}^{t} Z_{t, s}^{[2]} d W_{s} .
$$

Set

$$
Y_{t}^{[2]}=u_{2,2}\left(t, W_{\tau_{1}}, W_{t} ; t\right), Z_{t, s}^{[2]}=\frac{\partial u_{2,2}}{\partial y}\left(s, W_{\tau_{1}}, W_{s} ; t\right) .
$$

Applying Itô's formula to $u_{2,2}$ and the Brownian motion we get for $r \in\left[\tau_{1}, t\right]$ the following equation:

$$
\begin{aligned}
& u_{2,2}\left(r, W_{\tau_{1}}, W_{r} ; t\right)=u_{2,2}\left(\tau_{1}, W_{\tau_{1}}, W_{\tau_{1}} ; t\right) \\
& +\int_{\tau_{1}}^{r}\left[\frac{\partial u_{2,2}}{\partial s}\left(s, W_{\tau_{1}}, W_{s} ; t\right)+\frac{1}{2} \frac{\partial^{2} u_{2,2}}{\partial y^{2}}\left(s, W_{\tau_{1}}, W_{s} ; t\right)\right] d s+\int_{\tau_{1}}^{r} \frac{\partial u_{2,2}}{\partial y}\left(s, W_{\tau_{1}}, W_{s} ; t\right) d W_{s},
\end{aligned}
$$

where $u_{2,2}\left(\tau_{1}, W_{\tau_{1}}, W_{\tau_{1}} ; t\right)=\mathbb{E}\left[u_{2,2}\left(r, W_{\tau_{1}}, W_{r} ; t\right) \mid \mathcal{F}_{\tau_{1}}\right]$.

Comparing the previous equation and $(2.13)$, we can write

$$
\left\{\begin{array}{l}
\frac{\partial u_{2,2}}{\partial s}(s, x, y ; t)+\frac{1}{2} \frac{\partial^{2} u_{2,2}}{\partial y^{2}}(s, x, y ; t)=0 \\
u_{2,2}(t, x, y ; t)=u^{2,2}(t, x, y ; t)
\end{array}\right.
$$

from which it follows that

$$
\begin{aligned}
Y_{t}^{[2]}=u^{2,2}\left(t, W_{\tau_{1}}, W_{t} ; t\right)=u_{2,2}\left(t, W_{\tau_{1}}, W_{t} ; t\right) & \\
& =u_{2,2}\left(\tau_{1}, W_{\tau_{1}}, W_{\tau_{1}} ; t\right)+\int_{\tau_{1}}^{t} \frac{\partial u_{2,2}}{\partial y}\left(s, W_{\tau_{1}}, W_{s} ; t\right) d W_{s}
\end{aligned}
$$




$$
=\mathbb{E}\left[Y_{t}^{[2]} \mid \mathcal{F}_{\tau_{1}}\right]+\int_{\tau_{1}}^{t} Z_{t, s}^{[2]} d W_{s}
$$

Hence, 2.13) is satisfied.

For $(t, s) \in S_{2,1}$ consider $\varphi_{t}^{\tau_{1}}:=\mathbb{E}\left[Y_{t}^{[2]} \mid \mathcal{F}_{\tau_{1}}\right]$. In order to get 1.7 with $\mathcal{S}=0$, we require in view of (2.13) that

$$
\varphi_{t}^{\tau_{1}}=\mathbb{E}\left[Y_{t}^{[2]}\right]+\int_{0}^{\tau_{1}} Z_{t, s}^{[2]} d W_{s}
$$

Setting

$$
\varphi_{t}^{\tau_{1}}=u_{2,1}\left(\tau_{1}, W_{\tau_{1}} ; t\right), Z_{t, s}^{[2]}=\frac{\partial u_{2,1}}{\partial z}\left(s, W_{s} ; t\right),
$$

from Itô's formula we get the next system of PDE:

$$
\left\{\begin{array}{l}
\frac{\partial u_{2,1}}{\partial s}(s, z ; t)+\frac{1}{2} \frac{\partial^{2} u_{2,1}}{\partial z^{2}}(s, z ; t)=0 \\
u_{2,1}\left(\tau_{1}, z ; t\right)=u_{2,2}\left(\tau_{1}, z, z ; t\right)
\end{array}\right.
$$

since for $r \in\left[0, \tau_{1}\right]$ we have

$$
u_{2,1}\left(r, W_{r} ; t\right)=u_{2,1}\left(0, W_{0} ; t\right)+\int_{0}^{r} \frac{\partial u_{2,1}}{\partial z}\left(s, W_{s} ; t\right) d W_{s} .
$$

For $p \in\left[\tau_{1}, 1\right]$ one obtains

$$
\begin{aligned}
& u_{2,1}\left(0, W_{0} ; t\right)=\mathbb{E}\left[u_{2,1}\left(\tau_{1}, W_{\tau_{1}} ; t\right)\right]=\mathbb{E}\left[u_{2,2}\left(\tau_{1}, W_{\tau_{1}}, W_{\tau_{1}} ; t\right)\right] \\
& \quad=\mathbb{E}\left[u^{2,2}\left(p, W_{\tau_{1}}, W_{p} ; t\right)-\int_{\tau_{1}}^{p} \frac{\partial u_{2,2}}{\partial y}\left(s, W_{\tau_{1}}, W_{s} ; t\right) d W_{s}\right]=\mathbb{E}\left[u^{2,2}\left(p, W_{\tau_{1}}, W_{p} ; t\right)\right] .
\end{aligned}
$$

In particular, for $p=t$ we have that $u_{2,1}\left(0, W_{0} ; t\right)=\mathbb{E}\left[u^{2,2}\left(t, W_{\tau_{1}}, W_{t} ; t\right)\right]=$ $\mathbb{E}\left[Y_{t}^{[2]}\right]$, and

$$
\varphi_{t}^{\tau_{1}}=\mathbb{E}\left[Y_{t}^{[2]}\right]+\int_{0}^{\tau_{1}} Z_{t, s}^{[2]} d W_{s}
$$

At last for $(t, s) \in S_{1,1}$ we obtain from (1.7) the equation

$$
Y_{t}^{[2]}=\mathbb{E}\left[Y_{t}^{[2]}\right]+\int_{0}^{t} Z_{t, s}^{[2]} d W_{s}
$$




\section{PROOFS}

for a.e. $t \in\left[0, \tau_{1}\right]$.

Setting

$$
Y_{t}^{[2]}=u_{1,1}\left(t, W_{t} ; t\right), Z_{t, s}^{[2]}=\frac{\partial u_{1,1}}{\partial z}\left(s, W_{s} ; t\right),
$$

Itô's formula gives

$$
\left\{\begin{array}{l}
\frac{\partial u_{1,1}}{\partial s}(s, z ; t)+\frac{1}{2} \frac{\partial^{2} u_{1,1}}{\partial z^{2}}(s, z ; t)=0 \\
u_{1,1}(t, z ; t)=u^{1,1}(t, z ; t)
\end{array}\right.
$$

Hence, the solution of 1.7 is given by

$$
\begin{aligned}
& Y_{t}^{[2]}= \begin{cases}u_{2,2}\left(t, W_{\tau_{1}}, W_{t} ; t\right), & t \in\left[\tau_{1}, 1\right] \\
u_{1,1}\left(t, W_{t} ; t\right) & t \in\left[0, \tau_{1}\right)\end{cases} \\
& Z_{t, s}^{[2]}= \begin{cases}\frac{\partial u_{2,2}}{\partial z}\left(s, W_{\tau_{1}}, W_{s} ; t\right) & ,(t, s) \in S_{2,2} \\
\frac{\partial u_{2,1}}{\partial z}\left(s, W_{s} ; t\right) & ,(t, s) \in S_{2,1} \\
\frac{\partial u_{1,1}}{\partial y}\left(s, W_{s} ; t\right) & ,(t, s) \in S_{1,1}\end{cases}
\end{aligned}
$$

Here, of course, 2.17) coincides with (2.11).

If the interval $[0,1]$ is divided into $m$ parts at the points $\tau_{1}, \ldots, \tau_{m}\left(0<\tau_{1}<\right.$ $\left.\ldots<\tau_{m}=1\right)$ then $Y_{t}^{[m]}$ and $Z_{t, s}^{[m]}$ are computed with the next algorithm:

Consider (2.4) and a partition of $[0,1]^{2}$ into $2 m-1+\frac{m(m+1)}{2}$ intervals as shown in Figure 2.3. Thus, one has to solve $2 m-1+\frac{m(m+1)}{2}$ PDEs on the following subsets:

$$
\begin{aligned}
S^{1, j}:=\left\{(t, s) \in[0,1]^{2} \mid 0 \leq t<\tau_{j-1}, \tau_{j-1} \leq s \leq \tau_{j}\right\}, j=2, \ldots, m, \\
S^{2, j}:=\left\{\begin{array}{l}
\left\{(t, s) \in[0,1]^{2} \mid \tau_{m-1} \leq t \leq \tau_{m}, \tau_{m-1} \leq s \leq \tau_{m}, t \leq s\right\}, j=m \\
\left\{(t, s) \in[0,1]^{2} \mid \tau_{j-1} \leq t<\tau_{j}, \tau_{j-1} \leq s \leq \tau_{j}, t \leq s\right\}, j=1, \ldots, m-1,
\end{array}\right. \\
S_{i, j}:=\left\{\begin{array}{l}
\left\{(t, s) \in[0,1]^{2} \mid \tau_{m-1} \leq t \leq \tau_{m}, \tau_{j-1} \leq s \leq \tau_{j}\right\}, i=m, j=1, \ldots, m-1, i \neq j, \\
\left\{(t, s) \in[0,1]^{2} \mid \tau_{i-1} \leq t<\tau_{i}, \tau_{j-1} \leq s \leq \tau_{j}\right\}, i=1, \ldots, m-1, j=1, \ldots, m, i \neq j, \\
\left\{(t, s) \in[0,1]^{2} \mid \tau_{m-1} \leq t \leq \tau_{m}, \tau_{m-1} \leq s \leq \tau_{m}, t \geq s\right\}, i=j=m, \\
\left\{(t, s) \in[0,1]^{2} \mid \tau_{i-1} \leq t<\tau_{i}, \tau_{j-1} \leq s \leq \tau_{j}, t \geq s\right\}, i=j=1, \ldots, m-1 .
\end{array}\right.
\end{aligned}
$$




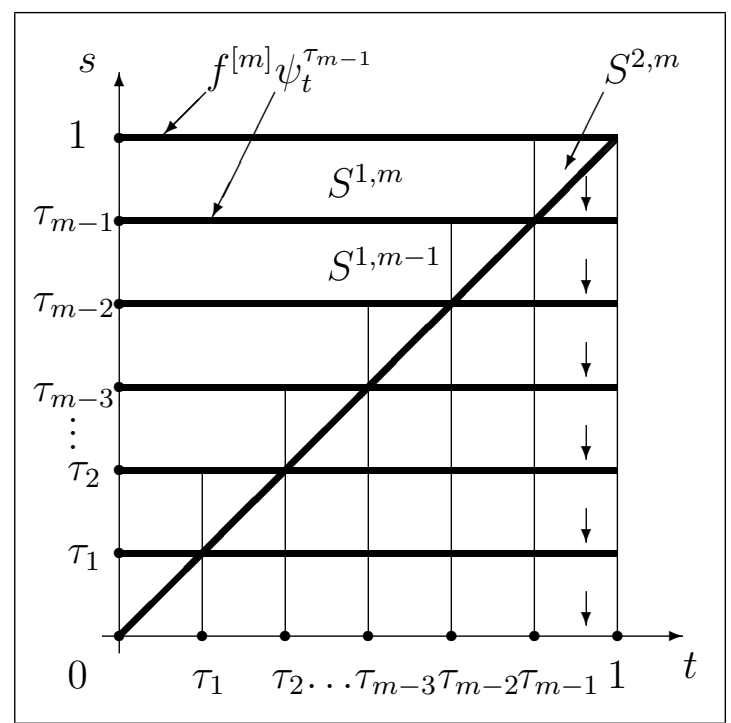

Figure 2.3: The algorithm of the construction in the general case. The terminal conditions of the systems of PDEs are defined on the thick lines.

We start in the right upper corner $S^{2, m}$. Denoting a vector $\left(x_{1}, \ldots, x_{m}\right)$ by $\boldsymbol{x}_{m}, u^{2, m}$ is given by the system

$$
\left\{\begin{array}{l}
\frac{\partial u^{2, m}}{\partial s}\left(s, \boldsymbol{x}_{m} ; t\right)+\frac{1}{2} \frac{\partial^{2} u^{2, m}}{\partial x_{m}^{2}}\left(s, \boldsymbol{x}_{m} ; t\right)=h^{[m]}\left(s, u^{2, m}\left(s, \boldsymbol{x}_{m} ; s\right)\right) \\
u^{2, m}\left(1, \boldsymbol{x}_{m} ; t\right)=f^{[m]}\left(\boldsymbol{x}_{m} ; t\right)
\end{array}\right.
$$

with $Y_{t}^{[m]}=u^{2, m}\left(t, \boldsymbol{W}_{\tau_{m-1}}, W_{t} ; t\right), Z_{t, s}^{[m]}=\frac{\partial u^{2, m}}{\partial x_{m}}\left(s, \boldsymbol{W}_{\tau_{m-1}}, W_{s} ; t\right)$.

With this we can solve on $S^{1, m}$

$$
\psi_{t}^{\tau_{m-1}}=f^{[m]}\left(\boldsymbol{W}_{\tau_{m}} ; t\right)-\int_{\tau_{m-1}}^{1} h^{[m]}\left(s, Y_{s}^{[m]}\right) d s-\int_{\tau_{m-1}}^{1} Z_{t, s}^{[m]} d W_{s}
$$

which can be translated to a parabolic equation $u^{1, m}$ with terminal condition $f^{[m]}$ and nonhomogeneous term being equal to $h^{[m]}\left(s, u^{2, m}\left(s, \boldsymbol{x}_{m} ; s\right)\right)$.

The most right column of the lower triangle can be solved using the Mcondition (1.7). Namely, we write the solution on $S_{m, m}$ in the following way:

$$
Y_{t}^{[m]}=\mathbb{E}\left[Y_{t}^{[m]} \mid \mathcal{F}_{\tau_{m-1}}\right]+\int_{\tau_{m-1}}^{t} Z_{t, s}^{[m]} d W_{s}
$$

which can be translated with Itô's formula into an homogeneous parabolic equa- 


\section{PROOFS}

tion of the form

$$
\left\{\begin{array}{l}
\frac{\partial u_{m, m}}{\partial s}\left(s, \boldsymbol{x}_{m} ; t\right)+\frac{1}{2} \frac{\partial^{2} u_{m, m}}{\partial x_{m}^{2}}\left(s, \boldsymbol{x}_{m} ; t\right)=0 \\
u_{m, m}\left(t, \boldsymbol{x}_{m} ; t\right)=u^{2, m}\left(t, \boldsymbol{x}_{m} ; t\right) .
\end{array}\right.
$$

Iteratively $u_{m, i}$ on $S_{m, i}$ is given by an homogeneous parabolic equation of the form (2.19) with terminal condition $u_{m, i+1}$.

With this we computed $Y_{t}^{[m]}$ and $Z_{t, s}^{[m]}$ in the regions $S^{1, m}, S^{2, m}$ and $S_{m, j}, j=$ $1, \ldots, m$. The next step is to compute this solution in the regions $S^{1, m-1}, S^{2, m-1}$ and $S_{m-1, j}, j=1, \ldots, m-1$.

Observing that

$$
Y_{t}^{[m]}=\psi_{t}^{\tau_{m-1}}-\int_{t}^{\tau_{m-1}} h^{[m]}\left(s, Y_{s}^{[m]}\right) d s-\int_{t}^{\tau_{m-1}} Z_{t, s}^{[m]} d W_{s},
$$

we can compute $Y_{t}^{[m]}$ and $Z_{t, s}^{[m]}$ on $S^{1, m-1}, S^{2, m-1}$ and $S_{m-1, j}, j=1, \ldots, m-1$, in an analogous way as on $S^{1, m}, S^{2, m}$ and $S_{m, j}, j=1, \ldots, m$, with new boundary condition $\psi_{t}^{\tau_{m-1}}$ instead of $f^{[m]}$. Proceeding in this way we can find $Y_{t}^{[m]}, Z_{t, s}^{[m]}$ on the whole square.

\subsubsection{Regularity Problem for the Solutions of PDEs}

In this part we study the well-posedness of the PDEs from the previous subsection, i.e. we show that the PDE has a unique classical solution. In addition, we show that even higher order derivatives exist, are continuous and bounded. We will need these derivatives in the proof of Lemma 2.5.1.

Our regularity result for the solutions of PDEs constructed on the sets from Figure 2.2 is the following:

Theorem 2.5.3. Let for the BSVIE (2.4) the conditions from Lemma 2.5.1 on $f^{[m]}$ and $h^{[m]}$ be fulfilled and $l=m=2, k=T=1$. Then there exist unique classical solutions $u^{i_{1}, i_{2}}$ and $u_{j_{1}, j_{2}}, j_{1} \geq j_{2}$, defined on the subsets $S^{1, i_{2}}, S^{2, i_{2}}, S_{j_{1}, j_{2}}$ (see Figure 2.2$), i_{1}, i_{2}, j_{1}, j_{2} \in\{1,2\}$, belonging to $C_{b}^{\infty}\left([0,1] \times \mathbb{R}^{i_{2}} \times[0,1] ; \mathbb{R}\right)$ and $C_{b}^{\infty}\left([0,1] \times \mathbb{R}^{j_{2}} \times[0,1] ; \mathbb{R}\right)$, respectively.

Obviously one can extend the result to the general case:

Corollary 2.5.4. Let for the BSVIE (2.4) the conditions from Lemma 2.5.1 on $f^{[m]}$ and $h^{[m]}$ be fulfilled. Then there exist unique classical solutions $u^{i_{1}, i_{2}}$ and $u_{j_{1}, j_{2}}, j_{1} \geq j_{2}$, defined on the subsets $S^{1, i_{2}}, S^{2, i_{2}}, S_{j_{1}, j_{2}}$ (see Figure 2.3), $i_{1} \in\{1,2\}, i_{2}, j_{1}, j_{2} \in\{1, \ldots, m\}$, belonging to $C_{b}^{\infty}\left([0, T] \times \mathbb{R}^{i_{2} \cdot d} \times[0, T] ; \mathbb{R}^{k}\right)$ and $C_{b}^{\infty}\left([0, T] \times \mathbb{R}^{j_{2} \cdot d} \times[0, T] ; \mathbb{R}^{k}\right)$, respectively. 
Before proving Theorem 2.5.3 we show the next lemma:

Lemma 2.5.5. Let $g \in C_{b}^{\infty}\left([t, 1] \times \mathbb{R}^{3} ; \mathbb{R}\right)$ depending on the variables $(s, y, z, x)$ be a bounded function, $t \in[0,1]$. Then for all $(s, y)$ the unique bounded classical solution $v(s, y ; x)$ of

$$
\left\{\begin{array}{l}
\frac{\partial v}{\partial s}(s, y ; x)+\frac{1}{2} \frac{\partial^{2} v}{\partial y^{2}}(s, y ; x)=g(s, y, v(s, y ; x) ; x), \quad(s, y ; x) \in[t, 1] \times \mathbb{R} \times \mathbb{R} \\
v(1, y ; x)=0 .
\end{array}\right.
$$

is $\infty$-times differentiable in $x$ with uniformly bounded derivatives.

In addition, the mixed partial derivatives $\frac{\partial^{i_{1}+i_{2}+i_{3} v}}{\partial s^{i_{1}} \partial y^{i_{2} \partial x^{i_{3}}}}$ exist, are continuous and bounded for $i_{3} \geq 1$ and $i_{1}, i_{2} \in \mathbb{N}_{0}$.

Proof. We use some ideas from [1] to achieve the claimed differentiability of $v$ in the variable $x$. Namely, using Theorem 4.5, Chapter 7, from [36], the solution of 2.20 has for $x$ fixed and $(t, y) \in[0,1] \times \mathbb{R}$ the following representation:

$$
v(t, y ; x)=\mathbb{E}\left[Y_{t}^{x, y, t}\right] \equiv Y_{t}^{x, y, t},
$$

where $\left(Y_{s}^{x, y, t}, Z_{s}^{x, y, t}\right)$ is the unique adapted solution of the family of BSDEs

$$
\left\{\begin{array}{l}
Y_{s}^{x, y, t}=\int_{t}^{1} g\left(s, W_{s}^{t, y}, Y_{s}^{x, y, t} ; x\right) d s+\int_{t}^{1} Z_{s}^{x, y, t} d W_{s} \\
Y_{1}^{x, y, t}=0
\end{array}\right.
$$

with $W_{s}^{t, y}:=y+\left(W_{s}-W_{t}\right)$ being the unique solution $X_{s}^{t, y}$ of the following SDE:

$$
\left\{\begin{array}{l}
d X_{s}^{t, y}=d W_{s}, s \in[t, 1] \\
X_{t}^{t, y}=y
\end{array}\right.
$$

Theorem 2.2 in [1] states that if conditions $(\mathrm{C} 1)-(\mathrm{C} 3)$ below are fulfilled then $Y_{s}^{x, y, t}$ is a.s. continuous in $s$ for all $(y, t)$ and that $Y_{s}^{x, y, t}, Z_{s}^{x, y, t}$ are continuously differentiable in $x$.

(C1) The function $g:[0,1] \times \mathbb{R}^{3} \rightarrow \mathbb{R}$ with $(s, y, z, x) \mapsto g(s, y, z ; x)$ is a $\mathcal{B}([0,1]) \otimes$ $\mathcal{B}\left(\mathbb{R}^{3}\right)$ measurable function. Moreover, it is globally Lipschitz in $(y, z)$ and continuously differentiable in $(y, z ; x)$; for all $(s, y, z)$ the mapping $x \mapsto$ $g(s, y, z ; x)$ is differentiable and for all $x \in \mathbb{R}$ and all $r \geq 1$ it holds that

$$
\text { (i) } \lim _{x^{\prime} \rightarrow x} \mathbb{E}\left[\left(\int_{t}^{1}\left|g\left(s, y, Y_{s}^{x, y, t} ; x^{\prime}\right)-g\left(s, y, Y_{s}^{x, y, t} ; x\right)\right| d s\right)^{r}\right]=0 .
$$




\section{PROOFS}

(ii) $\lim _{x^{\prime} \rightarrow x} \mathbb{E}\left[\left(\int_{t}^{1}\left|\frac{\partial g}{\partial x}\left(s, y, Y_{s}^{x^{\prime}, y, t} ; x^{\prime}\right)-\frac{\partial g}{\partial x}\left(s, y, Y_{s}^{x, y, t} ; x\right)\right| d s\right)^{r}\right]=0$.

(C2) The random variables $Y_{1}^{x, y, t}$ are $\mathcal{F}_{1}$-measurable and for every compact set $\mathcal{K} \subset \mathbb{R}$ there exist a constant $c \in \mathbb{R}$ such that

$$
\sup _{x \in \mathcal{K}} \sup _{\omega \in \Omega}\left|Y_{1}^{x, y, t}(\omega)\right| \leq c
$$

for all $r \geq 1$ and $(y, t) \in \mathbb{R} \times[0,1]$ the mapping $\mathbb{R} \rightarrow \mathbb{L}_{\mathcal{F}_{1}}^{r}\left(\Omega ; \mathbb{R}^{k}\right), x \mapsto Y_{1}^{x, y, t}$ is differentiable.

(C3) For $x \in \mathbb{R}$ and $\hat{h} \neq 0$ let

$$
\zeta(x, \hat{h}):=\frac{1}{\hat{h}^{2}}\left[Y_{1}^{x+\hat{h}, y, t}-Y_{1}^{x, y, t}\right] .
$$

For all $r \geq 1$ there exist a constant $c>0$ such that for $x, x^{\prime} \in \mathbb{R}$ and $\hat{h}, \hat{h}^{\prime} \in \mathbb{R} \backslash\{0\}$

$$
\mathbb{E}\left[\left|Y_{1}^{x+\hat{h}, y, t}-Y_{1}^{x^{\prime}+\hat{h}^{\prime}, y, t}\right|^{2 r}+\left|\zeta(x, \hat{h})-\zeta\left(x^{\prime}, \hat{h}^{\prime}\right)\right|^{2 r}\right] \leq c\left(\left|x-x^{\prime}\right|^{2}+\left|\hat{h}-\hat{h}^{\prime}\right|^{2}\right)^{r} .
$$

In our case the terminal condition $Y_{1}^{x, y, t}=0$, so $(\mathrm{C} 2)$ and $(\mathrm{C} 3)$ are trivially fulfilled.

To show (i) from (C1) we use that $g$ is differentiable with uniformly bounded derivatives in all variables and, hence, is a globally Lipschitz function with Lipschitz constant $L_{1}$ in all variables. Therefore,

$$
\left|g\left(s, y, Y_{s}^{x, y, t} ; x^{\prime}\right)-g\left(s, y, Y_{s}^{x, y, t} ; x\right)\right| \leq L_{1}\left|x^{\prime}-x\right|
$$

and

$$
\lim _{x^{\prime} \rightarrow x} \mathbb{E}\left[\left(\int_{t}^{1}\left|g\left(s, y, Y_{s}^{x, y, t} ; x^{\prime}\right)-g\left(s, y, Y_{s}^{x, y, t} ; x\right)\right| d s\right)^{r}\right] \leq \lim _{x^{\prime} \rightarrow x} L_{2}\left|x^{\prime}-x\right|^{r}=0
$$

for some $L_{2}>0$.

In order to prove condition (ii) let $\mu:=\mathbb{P} \otimes \lambda_{[0,1]}$ be the product measure of $\mathbb{P}$ and the Lebesgue measure $\lambda_{[0,1]}$. For each $r \geq 1$ we can apply Proposition 5.1 from [10], namely, for $(y, t)$ fixed

$$
\mathbb{E}\left[\left(\sup _{s \in[t, 1]}\left|Y_{s}^{x^{\prime}, y, t}-Y_{s}^{x, y, t}\right|\right)^{r}\right] \leq C(r)\left(\mathbb{E}\left[\int_{t}^{1}\left|g\left(s, y, Y_{s}^{x, y, t} ; x^{\prime}\right)-g\left(s, y, Y_{s}^{x, y, t} ; x\right)\right| d s\right]\right)^{r},
$$


where $C(r)$ is a constant depending only on $r$. Hence,

$$
\lim _{x^{\prime} \rightarrow x} \mathbb{E}\left[\left(\sup _{s \in[t, 1]}\left|Y_{s}^{x^{\prime}, y, t}-Y_{s}^{x, y, t}\right|\right)^{r}\right]=0
$$

and, for all $s \in[t, 1], Y_{s}^{x^{\prime}, y, t}$ converges to $Y_{s}^{x, y, t}$ in probability relative to $\mu\left(x^{\prime} \rightarrow\right.$ $x$ ). Since the partial derivative $\frac{\partial g}{\partial x}(s, y, z ; x)$ is continuous and bounded function, we obtain that for $(y, t)$ fixed and all $s \in[t, 1]$

$$
\lim _{x^{\prime} \rightarrow x} \frac{\partial g}{\partial x}\left(s, y, Y_{s}^{x^{\prime}, y, t} ; x^{\prime}\right)=\frac{\partial g}{\partial x}\left(s, y, Y_{s}^{x, y, t} ; x\right)
$$

in probability relative to $\mu$. Thus, from Theorem 21.4 in [3], $\frac{\partial g}{\partial x}\left(s, y, Y_{s}^{x^{\prime}, y, t} ; x^{\prime}\right)$ converges to $\frac{\partial g}{\partial x}\left(s, y, Y_{s}^{x, y, t} ; x\right)$ in $r$-th order mean relative to $\mu$ by the boundness of $\frac{\partial g}{\partial x}$, and it holds that

$$
\begin{aligned}
\lim _{x^{\prime} \rightarrow x} \mathbb{E}\left[\left(\int_{t}^{1}\left|\frac{\partial g}{\partial x}\left(s, y, Y_{s}^{x^{\prime}, y, t} ; x^{\prime}\right)-\frac{\partial g}{\partial x}\left(s, y, Y_{s}^{x, y, t} ; x\right)\right| d s\right)^{r}\right] \\
\leq \lim _{x^{\prime} \rightarrow x} \mathbb{E}\left[\iint_{t}^{1}\left|\frac{\partial g}{\partial x}\left(s, y, Y_{s}^{x^{\prime}, y, t} ; x^{\prime}\right)-\frac{\partial g}{\partial x}\left(s, y, Y_{s}^{x, y, t} ; x\right)\right|^{r} d s\right] \\
=\lim _{x^{\prime} \rightarrow x} \int_{\Omega \times[0,1]}\left|\mathbb{1}_{[t, 1]}(s)\left(\frac{\partial g}{\partial x}\left(s, y, Y_{s}^{x^{\prime}, y, t} ; x^{\prime}\right)-\frac{\partial g}{\partial x}\left(s, y, Y_{s}^{x, y, t} ; x\right)\right)\right|^{r} d \mu=0 .
\end{aligned}
$$

Hence, $v$ is differentiable in $x$.

Furthermore, denoting by $\left(\nabla Y_{s}^{x, y, t}, \nabla Z_{s}^{x, y, t}\right)$ the derivatives of $Y_{s}^{x, y, t}$ and $Z_{s}^{x, y, t}$ in $x$ they solve by Theorem 2.1 in [1] the BSDE

$$
\left\{\begin{array}{l}
\nabla Y_{s}^{x, y, t} \\
=\int_{t}^{1}\left[\frac{\partial g}{\partial x}\left(s, W_{s}^{t, y}, Y_{s}^{x, y, t} ; x\right)+\frac{\partial g}{\partial z}\left(s, W_{s}^{t, y}, Y_{s}^{x, y, t} ; x\right) \nabla Y_{s}^{x, y, t}\right] d s-\int_{t}^{1} \nabla Z_{s}^{x, y, t} d W_{s}, \\
\nabla Y_{1}^{x, y, t}=0
\end{array}\right.
$$

for $s \in[t, 1]$. In particular, $\tilde{v}(s, y ; x):=\nabla Y_{s}^{x, y, s}=\frac{\partial v}{\partial x}(s, y ; x)$ solves

$$
\left\{\begin{array}{l}
\frac{\partial \tilde{v}}{\partial s}(s, y ; x)+\frac{1}{2} \frac{\partial^{2} \tilde{v}}{\partial y^{2}}(s, y ; x) \\
\quad=\frac{\partial g}{\partial x}(s, y, v(s, y ; x) ; x)+\frac{\partial g}{\partial z}(s, y, v(s, y ; x) ; x) \tilde{v}(s, y ; x) \\
\tilde{v}(1, y ; x)=0 .
\end{array}\right.
$$




\section{PROOFS}

Denoting

$$
G_{1}(s, y ; x):=\frac{\partial g}{\partial x}(s, y, v(s, y ; x) ; x), \quad G_{2}(s, y ; x):=\frac{\partial g}{\partial z}(s, y, v(s, y ; x) ; x)
$$

and using Theorem 4.1, Chapter 7, from [36], the unique bounded solution of (2.23) is given explicitly by

$$
\tilde{v}(s, y ; x)=\mathbb{E}\left[-\int_{0}^{1-s} G_{1}\left(s+\theta, W_{\theta}^{s, y} ; x\right) e^{-\int_{0}^{\theta} G_{2}\left(\lambda+s, W_{\lambda}^{s, y} ; x\right) d \lambda} d \theta\right] .
$$

Note that the solution $\tilde{v}$ is indeed bounded as $G_{1}$ and $G_{2}$ are bounded. Consequently, $v$ can be differentiated a second time with second derivative

$$
\frac{\partial^{2} v}{\partial x^{2}}(s, y ; x)=\frac{\partial \tilde{v}}{\partial x}(s, y ; x)=\mathbb{E}\left[-\int_{0}^{1-s} \frac{\partial}{\partial x}\left(G_{1}\left(s+\theta, W_{\theta}^{s, y} ; x\right) e^{-\int_{0}^{\theta} G_{2}\left(\lambda+s, W_{\lambda}^{s, y} ; x\right) d \lambda}\right) d \theta\right]
$$

Continuing iteratively under the assumptions on the function $g$ we obtain that $v$ is $\infty$-times differentiable in $x$ with bounded derivatives.

In addition, as $\frac{\partial v}{\partial x}$ can be represented as in Equation (2.24), it follows that also the mixed partial derivatives exist, are continuous and bounded, if the function $g$ fulfills the assumptions stated in the Lemma.

Proof of Theorem 2.5.3. Writing the solution of 2.6 as

$$
u^{2,2}(s, x, y ; t)=\tilde{u}^{2,2}(s, x, y ; t)+\bar{u}^{2,2}(s, x, y),
$$

we get two Cauchy problems:

$$
\begin{aligned}
& \left\{\begin{array}{l}
\frac{\partial \tilde{u}^{2,2}}{\partial s}(s, x, y ; t)+\frac{1}{2} \frac{\partial^{2} \tilde{u}^{2,2}}{\partial y^{2}}(s, x, y ; t)=0, \\
\tilde{u}^{2,2}(1, x, y ; t)=f^{[2]}(x, y ; t)
\end{array}\right. \\
& \left\{\begin{array}{l}
\frac{\partial \bar{u}^{2,2}}{\partial s}(s, x, y)+\frac{1}{2} \frac{\partial^{2} \bar{u}^{2,2}}{\partial y^{2}}(s, x, y)=h^{[2]}\left(s, \tilde{u}^{2,2}(s, x, y ; s)+\bar{u}^{2,2}(s, x, y)\right) . \\
\bar{u}^{2,2}(1, x, y)=0
\end{array}\right.
\end{aligned}
$$

If we show that $\tilde{u}^{2,2}(s, x, y ; t) \in C_{b}^{\infty}\left([0,1] \times \mathbb{R}^{2} \times[0,1] ; \mathbb{R}\right)$ and $\bar{u}^{2,2}(s, x, y) \in$ $C_{b}^{\infty}\left([0,1] \times \mathbb{R}^{2} ; \mathbb{R}\right)$ then from 2.25$)$ it follows that $u^{2,2}(s, x, y ; t) \in C_{b}^{\infty}([0,1] \times$ $\left.\mathbb{R}^{2} \times[0,1] ; \mathbb{R}\right)$ 
Using Theorem 4.2, Chapter 4, from [15], the unique classical solution of (2.26) admits the following representation

$$
\tilde{u}^{2,2}(s, x, y ; t)=\int_{-\infty}^{+\infty} f^{[2]}(x, \xi ; t) \Phi(s, y, 1, \xi) d \xi
$$

for all $(x, t) \in \mathbb{R} \times\left[\tau_{1}, 1\right]$, where the density $\Phi$ is given by

$$
\Phi(s, y, \tilde{s}, \xi)=\frac{1}{\sqrt{2 \pi(\tilde{s}-s)}} e^{-\frac{(y-\xi)^{2}}{2(\tilde{s}-s)}}, \tau_{1} \leq s<\tilde{s}, y, \xi \in \mathbb{R} .
$$

With this we can compute for $l_{1}, l_{2}, l_{3} \in \mathbb{N}_{0}$

$$
\frac{\partial^{l_{1}+l_{2}+l_{3}} \tilde{u}^{2,2}}{\partial x^{l_{1}} \partial y^{l_{2}} \partial t^{l_{3}}}(s, x, y ; t)=\frac{1}{\sqrt{2 \pi(1-s)}} \int_{-\infty}^{+\infty} \frac{\partial^{l_{1}+l_{2}+l_{3}} f^{[2]}}{\partial x^{l_{1}} \partial y^{l_{2}} \partial t^{l_{3}}}(x, y-\xi ; t) e^{-\frac{\xi^{2}}{2(1-s)}} d \xi
$$

and together with $(2.26)$ it follows that $\tilde{u}^{2,2}(s, x, y ; t) \in C_{b}^{\infty}\left([0,1] \times \mathbb{R}^{2} \times[0,1] ; \mathbb{R}\right)$.

To see that $\bar{u}^{2,2}(s, x, y) \in C_{b}^{\infty}\left([0,1] \times \mathbb{R}^{2} ; \mathbb{R}\right)$ note the following: First it is clear from standard theory on PDEs, that for fixed $x$ the solution $\bar{u}^{2,2}(\cdot, x, \cdot) \in$ $C_{b}^{\infty}([0,1] \times \mathbb{R} ; \mathbb{R})$ under our assumptions on the generator $h^{[2]}$ and free term $f^{[2]}$. Secondly, using Lemma 2.5 .5 it holds that for $(s, y)$ fixed $\bar{u}^{2,2}(s, \cdot, y) \in C_{b}^{\infty}(\mathbb{R} ; \mathbb{R})$. In addition, all mixed derivatives involving $x$ in $(s, x, y)$ exist and are continuous, because the partial derivative in $x$-direction can be expressed in explicit form as in Equation (2.24).

Consequently, the function $u^{2,2}(s, x, y ; t)$ exhibits the required properties.

Due to the differentiability of the functions $f^{[2]}, h^{[2]}$ and $u^{2,2}$, using Theorem 4.1, Chapter 7, from [36], the unique classical solution of (2.9) is given by

$$
\begin{aligned}
u^{1,2}(s, x, y ; t) & =\int_{-\infty}^{+\infty} f^{[2]}(x, \xi ; t) \Phi(s, y, 1, \xi) d \xi \\
& \quad-\int_{0}^{1-s} \int_{-\infty}^{+\infty} h^{[2]}\left(s, u^{2,2}(s+\theta, x, \xi ; s+\theta)\right) \Phi(s, y, s+\theta, \xi) d \xi d \theta
\end{aligned}
$$

for all $x \in \mathbb{R}$.

Using assumption (M2) from Lemma 2.5.1, it follows that $u^{1,2}(s, x, y ; t) \in$ $C_{b}^{\infty}\left([0,1] \times \mathbb{R}^{2} \times[0,1] ; \mathbb{R}\right)$. In the same manner we show that $u^{1,1}(s, z ; t) \in$ $C_{b}^{\infty}([0,1] \times \mathbb{R} \times[0,1] ; \mathbb{R})$ writing $u^{1,1}$ as a sum of two functions solving easier PDEs similar to $\tilde{u}^{2,2}$ and $\bar{u}^{2,2}$. 


\section{PROOFS}

Notice that the solutions of the PDEs (2.14), 2.15), (2.16) represent Cauchy problems which are of the same type as the problem (2.26). We have the same regularity properties because the terminal condition of $u_{j_{1}, j_{2}}$ is $u^{i_{1}, i_{2}}$ which lies in $C_{b}^{\infty}\left([0,1] \times \mathbb{R}^{i_{2}} \times[0,1] ; \mathbb{R}\right)$. Hence, $u_{j_{1}, j_{2}}(s, x, y ; t) \in C_{b}^{\infty}\left([0,1] \times \mathbb{R}^{j_{2}} \times[0,1] ; \mathbb{R}\right)$

\subsubsection{Proof of Lemma 2.5.1}

Proof of Lemma 2.5.1. For simplicity, we show this lemma for $T=d=k=1$ and $m=2$, since the proof can be easily generalized.

In order to compute a discretization of $u^{i_{1}, i_{2}}, i_{1} \leq i_{2}\left(i_{1}, i_{2}=1,2\right)$, we consider $\left(s_{\eta}, x_{i}, y_{j} ; t_{\kappa}\right)=(\eta \Delta s, i \delta, j \delta ; \kappa \Delta t)$, where $\delta=\delta(n)=1 / \sqrt{n}, \Delta s=\Delta t=1 / n$.

From a Taylor expansion of $u^{2,2}$ we get the following recurrence equation:

$$
\begin{aligned}
& \frac{1}{2}\left(u^{2,2}\left(s_{\eta+1}, x_{i}, y_{j}+\delta ; t_{\kappa}\right)+u^{2,2}\left(s_{\eta+1}, x_{i}, y_{j}-\delta ; t_{\kappa}\right)\right) \\
&=u^{2,2}\left(s_{\eta}, x_{i}, y_{j} ; t_{\kappa}\right)+\frac{\partial u^{2,2}}{\partial s}\left(s_{\eta}, x_{i}, y_{j} ; t_{\kappa}\right) \delta^{2}+\frac{1}{2} \frac{\partial^{2} u^{2,2}}{\partial y^{2}}\left(s_{\eta}, x_{i}, y_{j} ; t_{\kappa}\right) \delta^{2} \\
&+ \frac{1}{4}\left(\frac{\partial^{2} u^{2,2}}{\partial y^{2}}\left(s_{\eta}^{+}, x_{i}, y_{j}^{+} ; t_{\kappa}\right) \delta^{2}-\frac{\partial^{2} u^{2,2}}{\partial y^{2}}\left(s_{\eta}, x_{i}, y_{j} ; t_{\kappa}\right) \delta^{2}\right) \\
&+\frac{1}{2} \frac{\partial^{2} u^{2,2}}{\partial y \partial s}\left(s_{\eta}^{+}, x_{i}, y_{j}^{+} ; t_{\kappa}\right) \delta^{3}+\frac{1}{4} \frac{\partial^{2} u^{2,2}}{\partial s^{2}}\left(s_{\eta}^{+}, x_{i}, y_{j}^{+} ; t_{\kappa}\right) \delta^{4} \\
&+\frac{1}{4}\left(\frac{\partial^{2} u^{2,2}}{\partial y^{2}}\left(s_{\eta}^{-}, x_{i}, y_{j}^{-} ; t_{\kappa}\right) \delta^{2}-\frac{\partial^{2} u^{2,2}}{\partial y^{2}}\left(s_{\eta}, x_{i}, y_{j} ; t_{\kappa}\right) \delta^{2}\right) \\
&+\frac{1}{2} \frac{\partial^{2} u^{2,2}}{\partial y \partial s}\left(s_{\eta}^{-}, x_{i}, y_{j}^{-} ; t_{\kappa}\right) \delta^{3}+\frac{1}{4} \frac{\partial^{2} u^{2,2}}{\partial s^{2}}\left(s_{\eta}^{-}, x_{i}, y_{j}^{-} ; t_{\kappa}\right) \delta^{4} \\
&= u^{2,2}\left(s_{\eta}, x_{i}, y_{j} ; t_{\kappa}\right)+h^{[2]}\left(s_{\eta}, u^{2,2}\left(s_{\eta}, x_{i}, y_{j} ; s_{\eta}\right)\right) \Delta s+O\left(\delta^{3}\right)
\end{aligned}
$$

for appropriate values $\left(s_{\eta}^{ \pm}, y_{j}^{ \pm}\right) \in\left[s_{\eta}, s_{\eta} \pm \delta^{2}\right] \times\left[y_{j}, y_{j} \pm \delta\right]$ and with $u^{2,2}\left(n, x_{i}, y_{j} ; t_{\kappa}\right)=$ $f^{[2]}\left(x_{i}, y_{j} ; t_{\kappa}\right)$, since

$$
\begin{aligned}
\left|\frac{\partial^{2} u^{2,2}}{\partial y^{2}}\left(s_{\eta}^{ \pm}, x_{i}, y_{j}^{ \pm} ; t_{\kappa}\right) \delta^{2}-\frac{\partial^{2} u^{2,2}}{\partial y^{2}}\left(s_{\eta}, x_{i}, y_{j} ; t_{\kappa}\right) \delta^{2}\right| \\
\leq\left\|\frac{\partial^{3} u^{2,2}}{\partial y^{3}}\right\|_{\infty} \delta^{3}+\left\|\frac{\partial^{3} u^{2,2}}{\partial y^{2} \partial s}\right\|_{\infty} \delta^{4}
\end{aligned}
$$

and the involved partial derivatives exist and are uniformly bounded in accordance with the assumptions, see Theorem 2.5.3. 
This motivates us to define $U_{n}^{2,2}(\eta, i, j ; \kappa)$ for $k_{1}=\left\lfloor\tau_{1} n\right\rfloor$ by

$$
\left\{\begin{array}{l}
\frac{1}{2}\left(U_{n}^{2,2}(\eta+1, i, j+1 ; \kappa)+U_{n}^{2,2}(\eta+1, i, j-1 ; \kappa)\right) \\
=U_{n}^{2,2}(\eta, i, j ; \kappa)+h^{[2]}\left(\eta, U_{n}^{2,2}(\eta, i, j ; \eta)\right) \frac{1}{n} \\
U_{n}^{2,2}(n, i, j ; \kappa)=f^{[2]}\left(x_{i}, y_{j} ; t_{\kappa}\right)
\end{array}\right.
$$

where $\kappa=n, \ldots, k_{1}, \eta=n-1, \ldots, \kappa, i, j \in \mathbb{Z}$.

Using Lipschitz condition on $h$, the previous difference equation has a unique solution for large $n$.

Similar, we approximate 2.9 and 2.10):

$$
\begin{aligned}
& \left\{\begin{array}{l}
\frac{1}{2}\left(U_{n}^{1,2}(\eta+1, i, j+1 ; \kappa)+U_{n}^{1,2}(\eta+1, i, j-1 ; \kappa)\right) \\
=U_{n}^{1,2}(\eta, i, j ; \kappa)+h^{[2]}\left(\eta, U_{n}^{2,2}(\eta, i, j ; \eta)\right) \frac{1}{n} \\
U_{n}^{1,2}(n, i, j ; \kappa)=f^{[2]}\left(x_{i}, y_{j} ; t_{\kappa}\right), \kappa=k_{1}, \ldots, 0, \eta=n-1, \ldots, k_{1}, i, j \in \mathbb{Z}
\end{array}\right. \\
& \left\{\begin{array}{l}
\frac{1}{2}\left(U_{n}^{1,1}(\eta+1, j+1 ; \kappa)+U_{n}^{1,1}(\eta+1, j-1 ; \kappa)\right) \\
=U_{n}^{1,1}(\eta, j ; \kappa)+h^{[2]}\left(\eta, U_{n}^{1,1}(\eta, j ; \eta)\right) \frac{1}{n} \\
U_{n}^{1,1}\left(k_{1}, j ; \kappa\right)=U_{n}^{1,2}\left(k_{1}, j, j ; \kappa\right), \kappa=k_{1}, \ldots, 0, \eta=k_{1}-1, \ldots, \kappa, j \in \mathbb{Z}
\end{array}\right.
\end{aligned}
$$

One easily shows that for

$$
Y_{t_{\eta}, t_{\kappa}}^{(n),[2]}= \begin{cases}U_{n}^{2,2}\left(\eta, W_{\tau_{1}^{(n)}}^{(n)}, W_{t_{\eta}}^{(n)} ; \kappa\right) & , \kappa=n, \ldots, k_{1}, \eta=n, \ldots, \kappa \\ U_{n}^{1,2}\left(\eta, W_{\tau_{1}^{(n)}}^{(n)}, W_{t_{\eta}}^{(n)} ; \kappa\right) & , \kappa=k_{1}, \ldots, 0, \eta=n, \ldots, k_{1} \\ U_{n}^{1,1}\left(\eta, W_{t_{\eta}}^{(n)} ; \kappa\right) & , \quad \kappa=k_{1}, \ldots, 0, \eta=k_{1}, \ldots, \kappa\end{cases}
$$

we have that

$$
Y_{t_{\eta}}^{(n),[2]}:=Y_{t_{\eta}, t_{\eta}}^{(n),[2]}=\mathbb{E}\left[f^{[2]}\left(W_{\tau_{1}^{(n)}}^{(n)}, W_{1}^{(n)} ; t_{\eta}\right)-\frac{1}{n} \sum_{\lambda=\eta}^{n-1} h^{[2]}\left(t_{\lambda}, Y_{t_{\lambda}, t_{\lambda}}^{(n),[2]}\right) \mid \mathcal{F}_{t_{\eta}}^{(n)}\right]
$$

and, hence,

$$
Y_{t_{\eta}}^{(n),[2]}= \begin{cases}U_{n}^{2,2}\left(\eta, W_{\tau_{1}^{(n)}}^{(n)}, W_{t_{\eta}}^{(n)} ; \eta\right) & , t_{\eta} \in\left[\tau_{1}^{(n)}, 1\right] \\ U_{n}^{1,1}\left(\eta, W_{t_{\eta}}^{(n)} ; \eta\right) & , t_{\eta} \in\left[0, \tau_{1}^{(n)}\right) .\end{cases}
$$

$\left(\begin{array}{l}\text { Note that by Remark } 2.5 .2 \text { Equation } 2.5 \text { has a unique solution for } \\ m=2 \text {, because } f^{[2]}\left(W_{\tau_{1}}, W_{1} ; t\right) \text { is } \mathcal{F}_{1} \text {-measurable for all } t \in[0,1] \text { and, } \\ \text { therefore, } f^{[2]}\left(W_{\tau_{1}^{(n)}}^{(n)}, W_{1}^{(n)} ; t_{i}\right) \text { is } \mathcal{F}_{t_{n}}^{(n)} \text {-measurable for all } i=0, \ldots, n .\end{array}\right)$ 


\section{PROOFS}

Now we want to obtain an upper bound for

$$
\theta_{n}^{2,2}(\eta):=\sup _{i, j \in \mathbb{Z}}\left|u^{2,2}\left(\eta \Delta s, x_{i}, y_{j} ; \kappa \Delta s\right)-U_{n}^{2,2}(\eta, i, j ; \kappa)\right|,
$$

$\kappa=n, \ldots, k_{1}, \eta=n-1, \ldots, \kappa$. From 2.30 and the definition of $U^{2,2}$, see (2.31), we find a constant $C_{1}$ with

$$
\theta_{n}^{2,2}(\eta) \leq \gamma\left[\theta_{n}^{2,2}(\eta+1)+\frac{C_{1}}{n \sqrt{n}}\right], \gamma=\frac{1}{1-L \Delta s},
$$

which yields the inequality

$$
\theta_{n}^{2,2}(\eta) \leq \gamma^{n-\eta} \theta_{n}^{2,2}(n)+\frac{C_{1}}{n \sqrt{n}} \sum_{p=1}^{n-\eta} \gamma^{p}=\gamma^{n-\eta} \theta_{n}^{2,2}(n)+\frac{C_{1}}{n \sqrt{n}} \gamma \frac{\gamma^{n-\eta}-1}{\gamma-1}
$$

and, therefore,

$$
\max _{k_{1} \leq \kappa \leq n} \max _{\kappa \leq \eta \leq n-1} \theta_{n}^{2,2}(\eta) \leq e^{2 L} \theta_{n}^{2,2}(n)+\frac{C_{1}}{n \sqrt{n}} \gamma \frac{e^{2 L}-1}{\gamma-1}=\frac{C_{1}\left(e^{2 L}-1\right)}{L \sqrt{n}}, \theta_{n}^{2,2}(n)=0 .
$$

Analogously, we find a constant $C_{2}$, such that

$$
\theta_{n}^{1,2}(\eta):=\sup _{i, j \in \mathbb{Z}}\left|u^{1,2}\left(\eta \Delta s, x_{i}, y_{j} ; \kappa \Delta s\right)-U_{n}^{1,2}(\eta, i, j ; \kappa)\right| \leq \frac{C_{1}\left(e^{2 L}-1\right)+C_{2}}{\sqrt{n}},
$$

where $\kappa=k_{1}, \ldots, 0, \eta=n-1, \ldots, k_{1}$, and

$$
\max _{0 \leq \kappa \leq k_{1}} \max _{n-1 \leq \eta \leq k_{1}} \theta_{n}^{1,2}(\eta) \leq \frac{C_{1}\left(e^{2 L}-1\right)+C_{2}}{\sqrt{n}} .
$$

At last consider

$$
\theta_{n}^{1,1}(\eta):=\sup _{i, j \in \mathbb{Z}}\left|u^{1,1}\left(\eta \Delta s, y_{j} ; \kappa \Delta s\right)-U_{n}^{1,1}(\eta, j ; \kappa)\right|,
$$

$\kappa=k_{1}, \ldots, 0, \eta=k_{1}-1, \ldots, \kappa$. Using the fact that $\frac{k_{1}}{n}=\frac{\left\lfloor\tau_{1} n\right\rfloor}{n} \leq \tau_{1}$ and denoting by $K^{1, i}:=C^{1, i}\left|\tau_{1}-k_{1} \Delta s\right|$, where $C^{1, i}$ is a bound for the derivatives of the function $u^{1, i}$ (in particular, for the derivatives involving $x$ ), $i=1,2$, for $\eta=k_{1}$ we get

$$
\left|u^{1,1}\left(\tau_{1}, y_{j} ; \kappa \Delta s\right)\right|-K^{1,1} \leq\left|u^{1,1}\left(k_{1} \Delta s, y_{j} ; \kappa \Delta s\right)\right| \leq\left|u^{1,1}\left(\tau_{1}, y_{j} ; \kappa \Delta s\right)\right|+K^{1,1} .
$$

Also we know that $u^{1,1}\left(\tau_{1}, y_{j} ; \kappa \Delta s\right)=u^{1,2}\left(\tau_{1}, y_{j}, y_{j} ; \kappa \Delta s\right)$. Thus, we can obtain a similar estimation for $u^{1,2}$ :

$\left|u^{1,2}\left(k_{1} \Delta s, y_{j}, y_{j} ; \kappa \Delta s\right)\right|-K^{1,2}$ 


$$
\begin{aligned}
\leq \mid u^{1,2}\left(\tau_{1}, y_{j}, y_{j} ;\right. & \kappa \Delta s) \mid \\
& \leq\left|u^{1,2}\left(k_{1} \Delta s, y_{j}, y_{j} ; \kappa \Delta s\right)\right|+K^{1,2} .
\end{aligned}
$$

Note, we needed here that $u^{2,2}$ is sufficiently smooth in $x$-direction because $u^{1,2}$ depends on $u^{2,2}$, and we can estimate as above $\left|u^{1,2}\left(\tau_{1}, y_{j}, y_{j} ; \kappa \Delta s\right)\right|$ only if $u^{1,2}$ is sufficiently smooth in $x$-direction.

Hence,

$$
\begin{aligned}
& \theta_{n}^{1,1}\left(k_{1}\right)=\sup _{i, j \in \mathbb{Z}}\left|u^{1,1}\left(k_{1} \Delta s, y_{j} ; \kappa \Delta s\right)-U_{n}^{1,2}\left(k_{1}, j, j ; \kappa\right)\right| \\
& \leq \theta_{n}^{1,2}\left(k_{1}\right)+2 C\left(\tau_{1}-k_{1} \Delta s\right) \leq \frac{C_{1}\left(e^{2 L}-1\right)+C_{2}+2 C}{\sqrt{n}},
\end{aligned}
$$

where $C:=\max _{i} C^{1, i}$ for all $i=1,2$.

Finally, for $k_{1}-1 \leq \eta \leq \kappa$ we can find a constant $C_{3}$

$$
\theta_{n}^{1,1}(\eta) \leq \gamma\left[\theta_{n}^{1,1}(\eta+1)+\frac{C_{3}}{n \sqrt{n}}\right]
$$

which yields the upper bound

$$
\begin{aligned}
\max _{0 \leq \kappa \leq k_{1}} \max _{k_{1}-1 \leq \eta \leq \kappa} \theta_{n}^{1,1}(\eta) \leq e^{2 L} \theta_{n}^{1,1}\left(k_{1}\right)+\frac{C_{3}\left(e^{2 L}-1\right)}{L \sqrt{n}} & \\
& \leq\left(e^{2 L}\left(C_{2}+2 C\right)+\frac{\left(e^{2 L}-1\right)\left(L e^{2 L} C_{1}+C_{3}\right)}{L}\right) \frac{1}{\sqrt{n}} .
\end{aligned}
$$

Using a suitable constant $A$, we get the following estimate:

$$
\max _{\eta}\left\{\theta_{n}^{2,2}(\eta), \theta_{n}^{1,1}(\eta)\right\} \leq \frac{A}{\sqrt{n}}
$$

With this the weak convergence of $\left(Y^{(n),[2]}\right)_{n \in \mathbb{N}}$ to $Y^{[2]}$ in the Skorokhod topology follows from Remark 2.5.2, i.e. the tightness of the sequence $\left(Y^{(n),[2]}\right)_{n \in \mathbb{N}}$, as from 2.33) follows that the finite dimensional distributions converge weakly. To see this, remark that for any $0 \leq \tilde{t}_{1}<\ldots<\tilde{t}_{j_{0}-1}<\tau_{1} \leq \tilde{t}_{j_{0}}<\ldots \tilde{t}_{p} \leq T$, $p \in \mathbb{N}, j_{0} \in 1, \ldots, p+1$, the finite dimensional distributions

$$
\begin{aligned}
\left(u^{1,1}\left(\tilde{t}_{1}, W_{\tilde{t}_{1}}^{(n)} ; \tilde{t}_{1}\right), \ldots, u^{1,1}\left(\tilde{t}_{j_{0}-1}, W_{\tilde{t}_{j_{0}-1}}^{(n)} ; \tilde{t}_{j_{0}-1}\right),\right. & \\
& \left.u^{2,2}\left(\tilde{t}_{j_{0}}, W_{\tau_{1}}^{(n)}, W_{\tilde{t}_{j_{0}}}^{(n)} ; \tilde{t}_{j_{0}}\right), \ldots, u^{2,2}\left(\tilde{t}_{p}, W_{\tau_{1}}^{(n)}, W_{\tilde{t}_{p}}^{(n)} ; \tilde{t}_{p}\right)\right)^{*}
\end{aligned}
$$




\section{PROOFS}

converge weakly to

$$
\begin{aligned}
& \left(u^{1,1}\left(\tilde{t}_{1}, W_{\tilde{t}_{1}} ; \tilde{t}_{1}\right), \ldots, u^{1,1}\left(\tilde{t}_{j_{0}-1}, W_{\tilde{t}_{j_{0}-1}} ; \tilde{t}_{j_{0}-1}\right),\right. \\
& \left.\quad u^{2,2}\left(\tilde{t}_{j_{0}}, W_{\tau_{1}}, W_{\tilde{t}_{j_{0}}} ; \tilde{t}_{j_{0}}\right), \ldots, u^{2,2}\left(\tilde{t}_{p}, W_{\tau_{1}}, W_{\tilde{t}_{p}} ; \tilde{t}_{p}\right)\right)^{*}=\left(Y_{\tilde{t}_{1}}^{[2]}, \ldots, Y_{\tilde{t}_{p}}^{[2]}\right)^{*},
\end{aligned}
$$

because of Donsker's theorem and the continuous mapping theorem applied to the continuous functions $u^{1,1}$ and $u^{2,2}$. In addition, it follows from (2.33) that

$$
\left(Y_{\tilde{t}_{1}}^{(n),[2]}, \ldots, Y_{\tilde{t}_{p}}^{(n),[2]}\right)^{*}-\left(u^{1,1}\left(\tilde{t}_{1}, W_{\tilde{t}_{1}}^{(n)} ; \tilde{t}_{1}\right), \ldots, u^{2,2}\left(\tilde{t}_{p}, W_{\tau_{1}}^{(n)}, W_{\tilde{t}_{p}}^{(n)} ; \tilde{t}_{p}\right)\right)^{*}
$$

converges to $(0, \ldots, 0)^{*}$ in probability and, hence, with Slutsky's theorem $\left(Y_{\tilde{t}_{1}}^{(n),[2]}, \ldots, Y_{\tilde{t}_{p}}^{(n),[2]}\right)^{*}$ converge weakly to $\left(Y_{\tilde{t}_{1}}^{[2]}, \ldots, Y_{\tilde{t}_{p}}^{[2]}\right)^{*}$.

\subsection{Proof of the Main Convergence Theorem}

Proof of Theorem 1.3.6. Let $m \in \mathbb{N}$ and $\tau^{\langle m\rangle}:=\left(\tau_{0}^{\langle m\rangle}, \ldots, \tau_{m}^{\langle m\rangle}\right)$ be a partition of $[0, T]\left(0=\tau_{0}^{\langle m\rangle}<\tau_{1}^{\langle m\rangle}<\ldots<\tau_{m}^{\langle m\rangle}=T\right)$.

For a point $\boldsymbol{x}:=\left(\boldsymbol{x}_{1}, \ldots, \boldsymbol{x}_{m}\right) \in \mathbb{R}^{l}$, where $\boldsymbol{x}_{i} \in \mathbb{R}^{d}, i=1, \ldots, m, m \in \mathbb{N}$, we define the linear interpolation $\mathbb{I}_{\tau^{\langle m\rangle}, \boldsymbol{x}}:[0, T] \rightarrow \mathbb{R}^{d}$ of $\tau^{\langle m\rangle}$ and $\boldsymbol{x}$ as

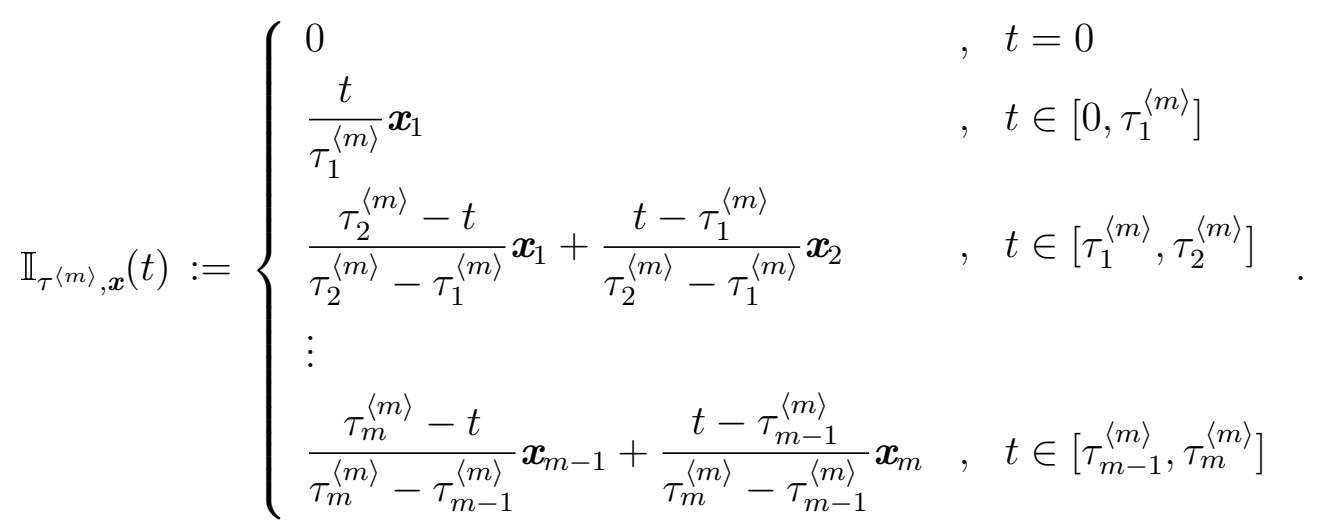

Define $f^{\langle m\rangle}: \mathbb{R}^{l} \times[0, T] \rightarrow \mathbb{R}^{k}$ by $f^{\langle m\rangle}(\boldsymbol{x} ; t):=f\left(\mathbb{I}_{\tau^{\langle m\rangle}, \boldsymbol{x}} ; t\right)$ and consider

$$
Y_{t}^{\langle m\rangle}=f^{\langle m\rangle}\left(\boldsymbol{W}_{\tau_{m}\langle m\rangle} ; t\right)-\int_{t}^{T} h\left(s, Y_{s}^{\langle m\rangle}\right) d s-\int_{t}^{T} Z_{t, s}^{\langle m\rangle} d W_{s}, t \in[0, T] .
$$

From Remark 2.5.2 Equation 2.34 has a unique adapted M-solution $\left(Y^{\langle m\rangle}, Z^{\langle m\rangle}\right)$. 


\subsection{Proof of the Main Convergence Theorem}

Assume, that $\left(\tau^{\langle m\rangle}\right)_{m \in \mathbb{N}}$ is a sequence of partitions, such that

$$
\lim _{m \rightarrow \infty} \max _{i \in\{0, \ldots, m-1\}}\left|\tau_{i+1}^{\langle m\rangle}-\tau_{i}^{\langle m\rangle}\right|=0
$$

Then from Lemma 2.3.1 it follows that $Y^{\langle m\rangle}$ converges to $Y$ in $L_{\mathbb{F}}^{2, \infty}$ for $m \rightarrow \infty$ as $f\left(\mathbb{I}_{\tau^{\langle m\rangle}, \boldsymbol{W}_{\tau_{m}\langle m\rangle}} ; t\right)$ converges to $f(W ; t)$ in $L_{\mathbb{F}}^{2, \infty}$.

In addition, as $f$ is bounded also $f^{\langle m\rangle}$ is bounded. Moreover $f^{\langle m\rangle}$ is Lipschitz with Lipschitz constant independent of $m$. Hence, we can approximate $f^{\langle m\rangle}$ and $h$ by a sequence of smooth functions $f^{\langle m\rangle,\{p\}}$ and $h^{\{p\}}$ with uniformly bounded derivatives, such that

$$
\lim _{p \rightarrow \infty} \sup _{m} \mathbb{E}\left[\sup _{s \in[0, T]}\left\|f^{\langle m\rangle,\{p\}}\left(\boldsymbol{W}_{\tau_{m}^{\langle m\rangle}} ; s\right)-f^{\langle m\rangle}\left(\boldsymbol{W}_{\tau_{m}^{\langle m\rangle}} ; s\right)\right\|_{2}^{2}\right]=0
$$

and

$$
\lim _{p \rightarrow \infty}\left\|h-h^{\{p\}}\right\|_{\infty}=0,
$$

because $Y^{\langle m\rangle}$ and $Y$ are uniformly bounded (due to Lemma 2.3.1).

With this we consider

$$
Y_{t}^{\langle m\rangle,\{p\}}=f^{\langle m\rangle,\{p\}}\left(\boldsymbol{W}_{\tau_{m}^{\langle m\rangle}} ; t\right)-\int_{t}^{T} h^{\{p\}}\left(s, Y_{s}^{\langle m\rangle,\{p\}}\right) d s-\int_{t}^{T} Z_{t, s}^{\langle m\rangle,\{p\}} d W_{s}, t \in[0, T] .
$$

Again from Remark 2.5.2 and Lemma 2.3.1 we follow, that there exists a unique M-solution $\left(Y^{\langle m\rangle,\{p\}}, Z^{\langle m\rangle,\{p\}}\right)$ of Equation (2.35) and that $Y^{\langle m\rangle,\{p\}}$ converges uniformly to $Y^{\langle m\rangle}$ as $p$ approaches $\infty$.

The free term $f^{\langle m\rangle,\{p\}}$ and the generator $h^{\{p\}}$ fulfill assumptions (M1) and (M2) of Lemma 2.5.1, therefore define $\tau^{(n),\langle m\rangle}:=\left(\tau_{0}^{(n),\langle m\rangle}, \ldots, \tau_{m}^{(n),\langle m\rangle}\right)$ with $\tau_{\lambda}^{(n),\langle m\rangle}:=\frac{\left\lfloor\tau_{\lambda}^{\langle m\rangle} n\right\rfloor}{n}$ for $\lambda=0, \ldots, m$, and consider

$$
\begin{aligned}
Y_{t_{i}}^{(n),\langle m\rangle,\{p\}}= & f^{\langle m\rangle,\{p\}}\left(\boldsymbol{W}_{\tau_{m}^{(n),\langle m\rangle}}^{(n)} ; t_{i}\right) \\
& -\frac{T}{n} \sum_{j=i}^{n-1} h^{\{p\}}\left(t_{j}, Y_{t_{j}}^{(n),\langle m\rangle,\{p\}}\right)-\sum_{j=i}^{n-1} Z_{t_{i}, t_{j}}^{(n),\langle m\rangle,\{p\}} \Delta W_{t_{j+1}}^{(n)} .
\end{aligned}
$$

Due to Theorem 1.3.4 there exists a unique M-solution $\left(Y^{(n),\langle m\rangle,\{p\}}, Z^{(n),\langle m\rangle,\{p\}}\right)$ of Equation (2.36) and from Lemma 2.5.1 it follows the weak convergence from $Y^{(n),\langle m\rangle,\{p\}}$ to $Y^{\langle m\rangle,\{p\}}$ when $n$ tends to $\infty$. 


\section{PROOFS}

Further, again from Theorem 1.3.4 one obtains that there exists a unique M-solution $\left(Y^{(n),\langle m\rangle}, Z^{(n),\langle m\rangle}\right)$ for

$$
Y_{t_{i}}^{(n),\langle m\rangle}=f^{\langle m\rangle}\left(\boldsymbol{W}_{\tau_{m}^{(n),\langle m\rangle}}^{(n)} ; t_{i}\right)-\frac{T}{n} \sum_{j=i}^{n-1} h\left(t_{j}, Y_{t_{j}}^{(n),\langle m\rangle}\right)-\sum_{j=i}^{n-1} Z_{t_{i}, t_{j}}^{(n),\langle m\rangle} \Delta W_{t_{j+1}}^{(n)}
$$

and from Lemma 2.3 .2 follows the uniform convergence of $Y^{(n),\langle m\rangle,\{p\}}$ to $Y^{(n),\langle m\rangle}$ as $p$ tends to $\infty$ (after $n$ ).

At last, again from Lemma 2.3.2 $Y^{(n),\langle m\rangle}$ converges uniformly to $Y^{(n)}$.

To sum up, if first $n$ converges to $\infty$, then $p$ and at last $m$, we obtain, that $Y^{(n)}$ converges weakly to $Y$ as from convergence in $L_{\mathbb{F}}^{2, \infty}$ and $L_{\mathbb{F}(n)}^{2, \infty}$ follows weak convergence in the Skorokhod topology.

More precisely, let $G: \mathbb{R}^{r} \rightarrow \mathbb{R}$ be a bounded continuous function, $r \geq 1$, and $0 \leq \tilde{t}_{1}<\ldots<\tilde{t}_{r} \leq T$ some partition of $[0, T]$, then we obtain

$$
\begin{aligned}
& \mathbb{E}\left[\left|G\left(Y_{\tilde{t}_{1}}, \ldots, Y_{\tilde{t}_{r}}\right)-G\left(Y_{\tilde{t}_{1}}^{(n)}, \ldots, Y_{\tilde{t}_{r}}^{(n)}\right)\right|\right] \\
& \leq \mathbb{E}\left[\left|G\left(Y_{\tilde{t}_{1}}, \ldots, Y_{\tilde{t}_{r}}\right)-G\left(Y_{\tilde{t}_{1}}^{\langle m\rangle}, \ldots, Y_{\tilde{t}_{r}}^{\langle m\rangle}\right)\right|\right] \\
& +\mathbb{E}\left[\left|G\left(Y_{\tilde{t}_{1}}^{\langle m\rangle}, \ldots, Y_{\tilde{t}_{r}}^{\langle m\rangle}\right)-G\left(Y_{\tilde{t}_{1}}^{\langle m\rangle,\{p\}}, \ldots, Y_{\tilde{t}_{r}}^{\langle m\rangle,\{p\}}\right)\right|\right] \\
& +\mathbb{E}\left[\left|G\left(Y_{\tilde{t}_{1}}^{\langle m\rangle,\{p\}}, \ldots, Y_{\tilde{t}_{r}}^{\langle m\rangle,\{p\}}\right)-G\left(Y_{\tilde{t}_{1}}^{(n),\langle m\rangle,\{p\}}, \ldots, Y_{\tilde{t}_{r}}^{(n),\langle m\rangle,\{p\}}\right)\right|\right] \\
& +\mathbb{E}\left[\left|G\left(Y_{\tilde{t}_{1}}^{(n),\langle m\rangle,\{p\}}, \ldots, Y_{\tilde{t}_{r}}^{(n),\langle m\rangle,\{p\}}\right)-G\left(Y_{\tilde{t}_{1}}^{(n),\langle m\rangle}, \ldots, Y_{\tilde{t}_{r}}^{(n),\langle m\rangle}\right)\right|\right] \\
& +\mathbb{E}\left[\left|G\left(Y_{\tilde{t}_{1}}^{(n),\langle m\rangle}, \ldots, Y_{\tilde{t}_{r}}^{(n),\langle m\rangle}\right)-G\left(Y_{\tilde{t}_{1}}^{(n)}, \ldots, Y_{\tilde{t}_{r}}^{(n)}\right)\right|\right] .
\end{aligned}
$$

The first two summands converge to zero, if first $p$ and then $m$, respectively, converges to $\infty$, because from convergence in $L_{\mathbb{F}}^{2, \infty}$, in particular, follows the convergence of the finite-dimensional distributions. The third summand converges due to Lemma 2.5.1, if $n$ tends to $\infty$. Analogously to the first two summands, the last two summands converge, if first $p$ and then $m$, respectively, tend to $\infty$. Together with the tightness of the sequences $\left(Y^{(n)}\right)_{n \in \mathbb{N}}$, we obtain that $\left(Y^{(n)}\right)_{n \in \mathbb{N}}$ converges weakly to $Y$. 


\section{Chapter 3}

\section{Numerical Example}

Consider for $(1.9)$ the following example:

$$
Y_{t}=t e^{\frac{1}{2}} \sin \left(W_{1}\right)+\int_{t}^{1} Y_{s} d s-\int_{t}^{1} Z_{t, s} d W_{s}, t \in[0,1]
$$

with $T=d=k=1$.

As conditions (V1) and (V2) hold, Equation (3.1) admits a unique solution $(Y, Z)$.

Notice that $Y_{t}=e^{\frac{t}{2}} \sin \left(W_{t}\right)$, since $e^{\frac{t}{2}} \sin \left(W_{t}\right)$ is a martingale and hence

$$
\mathbb{E}\left[t e^{\frac{1}{2}} \sin \left(W_{1}\right)+\int_{t}^{1} Y_{s} d s \mid \mathcal{F}_{t}\right]=t Y_{t}+(1-t) Y_{t}=Y_{t}
$$

Using 1.10 we find the discretization $Y_{t_{i}}^{(n)}$. If we interpolate piecewise constantly between values $Y_{t_{i}}^{(n)}$ and $Y_{t_{i+1}}^{(n)}$, from Theorem 1.3.6, we obtain that $Y^{(n)}$ converges weakly to $Y$. In particular, for every bounded continuous function $g: \mathbb{D}([0,1] ; \mathbb{R}) \rightarrow \mathbb{R}$, it holds, that

$$
\lim _{n \rightarrow \infty}\left(\mathbb{E}\left[g\left(Y^{(n)}\right)\right]-\mathbb{E}[g(Y)]\right)=0 .
$$

We examine the convergence by plotting the map

$$
n \mapsto\left(\mathbb{E}\left[\int_{0}^{1} \min \left\{Y_{s}^{2}, M\right\} d s\right]-\mathbb{E}\left[\frac{1}{n} \sum_{i=0}^{n-1} \min \left\{\left(Y_{t_{i}}^{(n)}\right)^{2}, M\right\}\right]\right)=: X^{(n)}, \quad M \geq e,
$$

see Figure 3.1, i.e. for the function $g(y):=\int_{0}^{1} \min \left\{y(s)^{2}, M\right\} d s$ with $y \in$ $\mathbb{D}([0,1] ; \mathbb{R})$. Applying Itô's formula to $e^{2 t} \sin ^{2}\left(W_{t}\right)$ and since $M \geq e$, we obtain that

$\mathbb{E}\left[\int_{0}^{1} \min \left\{Y_{s}^{2}, M\right\} d s\right]=\mathbb{E}\left[\int_{0}^{1} Y_{s}^{2} d s\right]=\int_{0}^{1} e^{s} \mathbb{E}\left[\sin ^{2}\left(W_{s}\right)\right] d s$ 


$$
=\frac{1}{2} \int_{0}^{1} e^{s}\left(1-e^{-2 s}\right) d s=\frac{(e-1)^{2}}{2 e}
$$

and the expected value in $X^{(n)}$ is computed as

$$
\mathbb{E}\left[\frac{1}{n} \sum_{i=0}^{n-1} \min \left\{\left(Y_{t_{i}}^{(n)}\right)^{2}, M\right\}\right]=\frac{1}{n} \sum_{i=0}^{n-1} \mathbb{E}\left[\min \left\{\left(Y_{t_{i}}^{(n)}\right)^{2}, M\right\}\right],
$$

where the solution of the DBSVIE $Y_{t_{i}}^{(n)}$ can be written as

$$
Y_{t_{i}}^{(n)}=\frac{n}{n-1} \mathbb{E}\left[t_{i} e^{\frac{1}{2}} \sin \left(W_{1}^{(n)}\right)+\frac{1}{n} \sum_{j=i+1}^{n-1} Y_{t_{j}}^{(n)} \mid \mathcal{F}_{t_{i}}^{(n)}\right]
$$

We plot $X^{(n)}$ in logarithmic scales on both the axes for $n=10,20,40, \ldots, 400$ with $M=10$, linear interpolated between the values. Using least squares method, we obtain that the slope of the map in Figure 3.1 is about -0.928 , which shows a good rate of convergence of our algorithm.

In addition, in Table 3.1 we show the convergence to zero of $Y_{0}^{(n)}$, which follows from the view of our solution for the problem (3.1). Because of computational rounding of numbers, in simulation we obtain some additional errors, which are not essential to show the required convergence. Note that $Y_{0}^{(n)}$ can take negative values as the random walk also does.

\begin{tabular}{|c||c|r|}
\hline $\mathrm{n}$ & $X^{(n)}$ & \multicolumn{1}{c|}{$Y_{0}^{(n)}$} \\
\hline 100 & 0.0339 & $0.0039 \times 10^{-16}$ \\
200 & 0.0172 & $-0.0078 \times 10^{-16}$ \\
300 & 0.0116 & $0.1067 \times 10^{-16}$ \\
400 & 0.0087 & $0.0503 \times 10^{-16}$ \\
\hline
\end{tabular}

Table 3.1: Values of $X^{(n)}$ and convergence of $Y_{0}^{(n)}$. 


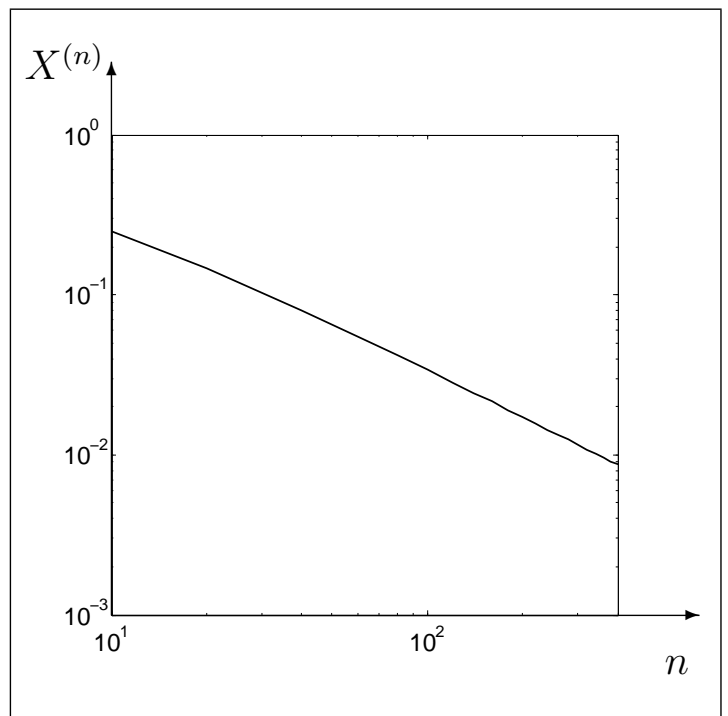

Figure 3.1: The speed of convergence of the numerical algorithm: it is plotted the map $n \mapsto X^{(n)}$ in logarithmic scales on both the axes for $n=10,20,40, \ldots, 400$ and linear interpolated between the values; the slope of the map is about -0.928 (calculated by least squares method). 
3. NUMERICAL EXAMPLE 


\section{Chapter 4}

\section{Outlook}

In this thesis we studied a numerical method for the BSVIE of the form

$$
Y_{t}=f(W ; t)-\int_{t}^{T} h\left(s, Y_{s}\right) d s-\int_{t}^{T} Z_{t, s} d W_{s}, t \in[0, T],
$$

which is defined on a filtered probability space $(\Omega, \mathcal{F}, \mathbb{F}, \mathbb{P})$, where $W$ is a $d$ dimensional Brownian motion and $\mathbb{F}$ the augmented Brownian filtration.

We showed that under certain regularity conditions on the coefficients in 4.1) the adapted M-solution $(Y, Z)$ can be approximated by a sequence $\left(Y^{(n)}, Z^{(n)}\right)_{n \in \mathbb{N}}$ of discrete BSVIEs driven by a binary random walk. Precisely, we proved that the sequence of discrete solutions $\left(Y^{(n)}\right)_{n \in \mathbb{N}}$ converges weakly to the continuous process $Y$ in the Skorokhod topology. For the proof we used the relationship of the M-solution of (4.1) to a non-standard system of quasilinear partial differential equation of parabolic type.

Next, we would like to discuss what parts of the numerical method still hold for general BSVIEs of the form (1.6) and what may fail. In the general form the function $h$ may depend not only on $s$ and $Y_{s}$, but also on $t, Z_{t, s}$ and $Z_{s, t}$.

(a) The implicit form of $Z^{(n)}$ holds also in the general case.

(b) If the generator $h$ depends on $t, Z_{t, s}$ or $Z_{s, t}$, we cannot explicitly construct the process $\hat{Y}_{t_{i}}^{(n)}$ with the fixed point technique given in Section 1.3 , since $Y_{t_{i}}^{(n)}$ cannot be expressed via 1.13 .

(c) We have shown the required regularity of the functions $u^{i_{1}, i_{2}}$ and $u_{j_{1}, j_{2}}$ (see Section 2.5.2 with an application of Theorem 2.2 from [1]. If the generator $h$ depends on $Z$ the derivative $\nabla Y_{s}^{x, y, t}$ (see Equation (2.22) ) of the process $Y_{s}^{x, y, t}$ (see Equation (2.21)) solves a family of BSDEs involving also the 


\section{OUTLOOK}

derivative of $Z$ in the generator and, hence, one cannot apply Theorem 2.2 from [1] to achieve the required regularity.

(d) If the generator $h$ depends on $Z_{s, t}$, the stepwise construction of the PDEs is not possible, as, for example, one cannot solve the corresponding Equation to (2.6) without solving the corresponding Equation to (2.14) and vice versa. More precisely, we do not obtain a system of two PDEs, where the second PDE depends on the first through the boundary condition, but a system of PDEs which depend of each other on one side through the boundary condition and on the other side through the inhomogeneous term. This makes the system more complicated to understand.

To conclude, we remark that our numerical method can also be applied for $S$-solutions of BSVIEs of the form (4.1). One calls a pair of processes $(Y, Z)$ a $S$-solution, if $Z_{t, s}$ is not defined by a martingale representation property, but equals $Z_{s, t}$ (see [31]). The proof of the corresponding Main Convergence Theorem is even easier, because one has to consider only the systems of PDEs defined on the upper triangle. 


\section{Bibliography}

[1] Ankirchner, S., Imkeller, P., And Gonçalo, D.R. Classical and variational differentiability of BSDEs with quadratic growth. Electr. Jour. of Probab., 12:1418-1453, 2007.

[2] Bally, V. Approximation scheme for solutions of BSDE. El Karoui., N., et al. (Edts), Backward stochastic differential equations, 364:177-191, 1997.

[3] Bauer, H. Maß- und Integrationstheorie. Walter de Gruyter, Berlin, 1992.

[4] Berger, M. And Mizel, V. Volterra equations with Itô integrals. Int. Eq., 2:187-245, 319-337, 1980.

[5] Billingsley, P. Convergence of probability measure. Wiley \& Sons, New York, 1968.

[6] Briand, P., Delyon, B., And Memin, J. Donsker-type theorem of BSDEs. Elect. Comm. in Probab., 6:1-14, 2001.

[7] Chevance, D. Numerical methods for backward stochastic differential equations. L.C.G. Rogers, et al. (Edts), Numerical methods in finance, University Press, Cambridge, pages 232-244, 1997.

[8] Douglas, J., Ma, J., And Protter, P. Numerical methods for forwardbackward stochastic differential equations. Ann. Appl. Probab., 6:940-968, 1996.

[9] El Karoui, N. Backward stochastic differential equations a general introduction. El Karoui., N., et al. (Edts), Backward stochastic differential equations, 364:7-26, 1997.

[10] El Karoui, N., Peng, S., And Quenez, M. C. Backward stochastic differential equations in finance. Math. Finance, 7:1-71, 1997.

[11] Friedman, A. Partial differential equations of parabolic type. Prentice-Hall, Englewood Cliffs, 1964. 
[12] Ikeda, N. And Watanabe, S. Stochastic differential equations and diffusion processes. North-Holland, Tokyo, 1989.

[13] Jacod, J., Memin, J., And Metivier, M. On tightness and stopping times. Stoch. Proc. Appl., 14:109-146, 1983.

[14] Jacod, J. And ShiRYAev, A.N. Limit theorems for stochastic processes. Springer-Verlag, Berlin, 1987.

[15] Karatzas, I. And Shreve, S.E. Brownian motion and stochastic calculus. Springer-Verlag, New York, 1988.

[16] Klebaner, F.C. Introduction to stochastic calculus with applications. Imperial College Press, London, 2005.

[17] Lin, J. Adapted solution of backward stochastic nonlinear Volterra integral equations. Stoch. Anal. Appl., 20:165-183, 2002.

[18] Ma, J., Protter, P., San Martin, J., And Torres, S. Numerical method for backward stochastic differential equations. Ann. Appl. Probab., 12:302-316, 2002.

[19] Ma, J., Protter, P., And Yong, J. Solving forward-backward stochastic differential equations explicitly - a four step scheme. Probab. Theory Relat. Fields, 98:339-359, 1994.

[20] Milstein, G. N. And Tretyakov, M.V. Numerical algorithms for forward-backward stochastic differential equations. SIAM J. Sci. Comput., 28:561-582, 2006.

[21] ØKsendal, B. Stochastic differential equations: an introduction with applications. Springer-Verlag, Berlin, 2000.

[22] Pardoux, E. And Peng, S. Adapted solution of a backward stochastic differential equation. Systems \& Control Letters, 14:55-61, 1990.

[23] Pardoux, E. And Peng, S. Backward stochastic differential equations and quasilinear parabolic partial differential equations. Lecture Notes in Control and Information Sciences, Springer, 176:200-217, 1992.

[24] Peng, S. A nonlinear Feynman-Kac formula and applications. Proc. Symposium of System Sciences and Control Theory, Chen \& Yong eds., pages 173-184, World Scientific, Singapore, 1992. 
[25] PENG, S. Nonlinear expectations, nonlinear evaluations and risk measures. Stoch. Methods in Fin., Lecture Notes in Math., Springer, 1856:165-253, 2004 .

[26] Pham, H. Continuous-time stochastic control and optimization with financial applications. Springer-Verlag, Berlin, 2009.

[27] Privault, N. Stochastic analysis in discrete and continuous settings. Springer-Verlag, Berlin, 2009.

[28] Prokhorov, Y.U. Convergence of stochastic processes and limit theorems in probability theory. Theory Probab. Appl., 1:157-214, 1956.

[29] Rouge, R. and El Karoui, N. Pricing via utility maximization and entropy. Math. Finance, 10(2):259-276, 2000.

[30] WANG, T. BSVIEs with stochastic lipschitz coefficients and applications in finance. Preprint, 2010.

[31] Wang, T. And Shi, Y. Symmetrical solutions of backward stochastic volterra integral equations and their applications. Preprint, 2009.

[32] Yong, J. Forward-backward stochastic differential equations and their applications. Springer-Verlag, Berlin, 1999.

[33] Yong, J. Backward stochastic volterra integral equations and some related problems. Stoch. Proc. Appl., 116:779-795, 2006.

[34] Yong, J. Continuous-time dynamic risk measures by backward stochastic volterra integral equations. Appl. Ann., 86:1429-1442, 2007.

[35] YoNG, J. Well-posedness and regularity of backward stochastic volterra integral equations. Probab. Theory Relat. Fields, 142:21-77, 2008.

[36] Yong, J. And Zhou, X.Y. Stochastic controls: Hamiltonian systems and HJB equations. Springer-Verlag, New York, 1999. 